\title{
Solving Local Equivalence Problems with the Equivariant Moving Frame Method ${ }^{\star}$
}

\author{
Francis VALIQUETTE \\ Department of Mathematics and Statistics, Dalhousie University, \\ Halifax, Nova Scotia, B3H 3J5, Canada \\ E-mail: francisv@mathstat.dal.ca \\ URL: http://www.mathstat.dal.ca/ francisv
}

Received July 21, 2012, in final form March 31, 2013; Published online April 05, 2013

http://dx.doi.org/10.3842/SIGMA.2013.029

\begin{abstract}
Given a Lie pseudo-group action, an equivariant moving frame exists in the neighborhood of a submanifold jet provided the action is free and regular. For local equivalence problems the freeness requirement cannot always be satisfied and in this paper we show that, with the appropriate modifications and assumptions, the equivariant moving frame constructions extend to submanifold jets where the pseudo-group does not act freely at any order. Once this is done, we review the solution to the local equivalence problem of submanifolds within the equivariant moving frame framework. This offers an alternative approach to Cartan's equivalence method based on the theory of $G$-structures.
\end{abstract}

Key words: differential invariant; equivalence problem; Maurer-Cartan form; moving frame

2010 Mathematics Subject Classification: 53A55; 58A15

In honor of Peter Olver's $60^{\text {th }}$ birthday. Thank you for your guidance.

\section{Introduction}

First introduced by the Estonian mathematician Martin Bartels and primarily developed by Élie Cartan, [1], the method of moving frames is a powerful tool for studying geometric properties of submanifolds under the action of a (pseudo-)group of transformations. In 1999, Fels and Olver proposed in [9] a new theoretical foundation to the method of moving frames. For a Lie group $G$ acting on the $n^{\text {th }}$ order jet space $\mathrm{J}^{n}(M, p)$ of $p$-dimensional submanifolds of $M$, a moving frame is a $G$-equivariant section of the trivial bundle $\mathrm{J}^{n}(M, p) \times G \rightarrow \mathrm{J}^{n}(M, p)$. This new framework to moving frames, now known as the equivariant moving frame method, possesses some appealing features. First, it decouples the moving frame theory from reliance on any form of frame bundle or connection and can thereby be applied to almost any type of group action. Secondly, every equivariant moving frame comes with an invariantization map that sends differential functions, differential forms, and vector fields to their invariant counterparts yielding a complete collection of (local) differential invariants, invariant differential forms, and invariant vector fields. In general, the invariantization map and the exterior differential do not commute, and this lack of commutativity is encapsulated in the universal recurrence formula which is at the heart of many new results in the field. For example, using this fundamental formula, Kogan and Olver were able to obtained in [16] a general group-invariant formula for the Euler-Lagrange equations of an invariant variational problem, while in [39] the same formula was used to show that the cohomology of the invariant Euler-Lagrange complex is isomorphic to the Lie algebra cohomology of its symmetry group. But more importantly, the universal recurrence formula is the key that

\footnotetext{
* This paper is a contribution to the Special Issue "Symmetries of Differential Equations: Frames, Invariants and Applications". The full collection is available at http://www.emis.de/journals/SIGMA/SDE2012.html
} 
unveils the structure of the algebra of differential invariants, $[9,13,28]$, essential to the solution of local equivalence problems.

Recently, the theory of equivariant moving frames was successfully extended to infinitedimensional Lie pseudo-group actions in [28, 29, 30]; opening the way to many new applications. The first application appeared in [6] where the algebra of differential invariants of the infinite-dimensional symmetry group of the Kadomtsev-Petviashvili equation was completely characterized. An application to the classification of Laplace invariants and the factorization of linear partial differential operator can be found in [36], and an adaptation of Vessiot's group foliation method using moving frames was proposed in [38] (see [34] for an alternative construction based on exterior differential systems). As a further application, in this paper we revisit the solution of the local equivalence problem of submanifolds under an infinite-dimensional Lie pseudo-group action using the equivariant moving frame formalism. Following Cartan, the solution is essentially obtained by constructing sufficiently many invariants so that one can distinguish inequivalent submanifolds. With the equivariant moving frame method, these invariants are easily constructed using the invariantization map and their signature manifold is completely characterized by the universal recurrence formula. Since the algorithms do not rely on the theory of exterior differential systems and $G$-structures, $[2,10,15,21,26]$, the solution based on the equivariant moving frame method offers an interesting alternative to Cartan's equivalence method of coframes.

To construct an equivariant moving frame in the neighborhood of a submanifold jet, the pseudo-group action must be (locally) free and regular. Unfortunately, given an equivalence problem, the freeness requirement cannot always be satisfied, and more often than not many interesting results occur at the submanifold jets where the action cannot be made free by prolongation. For example, it is well-known that a second order ordinary differential equation $u_{x x}=Q\left(x, u, u_{x}\right)$ is equivalent to $u_{x x}=0$ under a point transformation if and only if it admits an eight-dimensional symmetry group isomorphic to $\mathrm{SL}(3),[5,10,26,40]$. For such a differential equation, the pseudo-group of point transformations cannot act freely and the freeness assumption must be relaxed if one wants to obtain a complete solution of the local equivalence problem using the equivariant moving frame method. As one might expect, the idea is to modify the standard moving frame algorithms by incorporating the isotropy group into the constructions to obtain what we call a partial equivariant moving frame. Using (partial) moving frames we can solve a wide range of local equivalence problems, which includes equivalence problems between coframes. To illustrate the method we consider the local equivalence problem of second order ordinary differential equations under point transformations and contact transformations, and the simultaneous equivalence of a two-form and a vector field on $\mathbb{R}^{3}$. By revisiting these standard examples, our goal is to highlight some links between the (partial) equivariant moving frame approach and Cartan's method.

The solution of a local equivalence problem relies on the fundamental basis theorem (also known as the Lie-Tresse theorem) which states that, under appropriate regularity assumptions, the algebra of differential invariants on $\mathrm{J}^{\infty}(M, p)$ is locally generated by a finite set of differential invariants and exactly $p$ linearly independent invariant total derivative operators. Under the assumption that a pseudo-group action is regular and locally free on a dense open subbundle of $\mathrm{J}^{\infty}(M, p)$, a constructive proof of the fundamental basis theorem based on the equivariant moving frame method was recently given in [28]. In Section 5, we adapt the algebraic constructions introduced in [28] to cover pseudo-groups acting regularly and freely on invariant regular subbundles of $\mathrm{J}^{\infty}(M, p)$ and also consider the case of regular pseudo-groups acting non-freely on invariant regular subbundles of $\mathrm{J}^{\infty}(M, p)$. These adaptations are necessary to give a complete solution to the local equivalence problem of submanifolds.

Remark 1.1. The theory of infinite-dimensional Lie pseudo-groups relies on the Cartan-Kähler theorem, [2, 26], which requires analyticity. For this reason, all our constructions and results 
hold in the analytic category. Implicitly, all manifolds, maps, differential forms and vector fields are thus assumed to be analytic. For Lie pseudo-groups of finite type, in other words for local Lie group actions, analyticity can be replaced by smoothness.

Remark 1.2. Following the global notation convention used in $[9,28,29,30]$, given a map $\varphi: M \rightarrow N$ between two manifolds $M$ and $N$ we allow the domain of the map to be a proper open subset of the manifold $M: \operatorname{dom} \varphi \subset M$. Hence, while we use global notation throughout the exposition, all moving frame constructions and results should be understood to hold microlocally, i.e. on open subsets of the submanifold jet bundle $\mathrm{J}^{\infty}(M, p)$. Similarly, differential forms and vector fields on $\mathrm{J}^{\infty}(M, p)$ are assumed to be defined micro-locally.

\section{Structure equations}

Following $[7,29]$ we begin by recalling how the structure equations of a Lie pseudo-group are obtained from its infinitesimal data. As we will see in Section 4, the structure equations of an equivalence pseudo-group provide the link between the equivariant moving frame method and Cartan's moving frame approach.

\subsection{Diffeomorphism pseudo-group}

Let $M$ be an $m$-dimensional manifold. We denote by $\mathcal{D}=\mathcal{D}(M)$ the pseudo-group of all local diffeomorphisms of $M$. For each integer $0 \leq n \leq \infty$, let $\mathcal{D}^{(n)}$ denote the bundle formed by their $n^{\text {th }}$ order jets. For $k \geq n$, let $\widetilde{\pi}_{n}^{k}: \mathcal{D}^{(k)} \rightarrow \mathcal{D}^{(n)}$ denote the standard projection. Following Cartan, [3, 4], and the recent work of Olver and Pohjanpelto, [28, 29, 30], in some local coordinate system we use lower case letters, $z, x, u, \ldots$ for the source coordinates $\widetilde{\boldsymbol{\sigma}}(\varphi)=z=\left(z^{1}, \ldots, z^{m}\right) \in M$ of a local diffeomorphism $Z=\varphi(z)$ and corresponding upper case letters $Z, X, U, \ldots$ for the target coordinates $\widetilde{\boldsymbol{\tau}}(\varphi)=Z=\left(Z^{1}, \ldots, Z^{m}\right) \in M$. The local coordinates of the $n$-jet of a local diffeomorphism $\varphi$ are then given by $\mathrm{j}_{n} \varphi=\left(z, Z^{(n)}\right)$, where $z$ are the source coordinates and $Z^{(n)}$ denotes the collection of derivatives $Z_{B}^{a}=\partial^{k} \varphi^{a} / \partial z^{b^{1}} \cdots \partial z^{b^{k}}$ with $1 \leq a, b^{1}, \ldots, b^{k} \leq m$ and $0 \leq k=\# B \leq n$.

The diffeomorphism jet bundle $\mathcal{D}^{(\infty)}$ has the structure of a groupoid, [23]. The groupoid multiplication follows from the composition of local diffeomorphisms. For $\left.g^{(\infty)}\right|_{z}=\left.\mathrm{j}_{\infty} \varphi\right|_{z}$ and $\left.h^{(\infty)}\right|_{Z}=\left.\mathrm{j}_{\infty} \psi\right|_{Z}$ with $Z=\widetilde{\boldsymbol{\tau}}^{(\infty)}\left(\left.\mathrm{j}_{\infty} \varphi\right|_{z}\right)=\tilde{\boldsymbol{\sigma}}^{(\infty)}\left(\left.\mathrm{j}_{\infty} \psi\right|_{Z}\right)$, we have that $\left.\left(h^{(\infty)} \cdot g^{(\infty)}\right)\right|_{z}=$ $\left.\mathrm{j}_{\infty}(\psi \circ \varphi)\right|_{z}$. Throughout the paper, the diffeomorphism pseudo-group $\mathcal{D}$ acts on $\mathcal{D}^{(\infty)}$ by right multiplication:

$$
R_{\psi}\left(\left.\mathrm{j}_{\infty} \varphi\right|_{z}\right)=\left.\mathrm{j}_{\infty}\left(\varphi \circ \psi^{-1}\right)\right|_{\psi(z)},
$$

whenever defined.

The cotangent space $T^{*} \mathcal{D}^{(\infty)}$ naturally splits into horizontal and contact (groupoid) components. The horizontal subbundle is spanned by the right-invariant one-forms

$$
\sigma^{a}=d_{M} Z^{a}=\sum_{b=1}^{m} Z_{b}^{a} d z^{b}, \quad a=1, \ldots, m,
$$

while the contact subbundle is spanned by the (right-invariant) Maurer-Cartan forms

$$
\mu_{B}^{a}, \quad a=1, \ldots, m, \quad \# B \geq 0 .
$$

Their coordinate expressions are obtained by repeatedly applying the total derivative operators

$$
\mathbb{D}_{Z^{b}}=\sum_{a=1}^{m} W_{b}^{a} \mathbb{D}_{z^{a}}, \quad\left(W_{a}^{b}\right)=\left(Z_{a}^{b}\right)^{-1}
$$


to the order zero Maurer-Cartan forms

$$
\mu^{a}=d_{G} Z^{a}=d Z^{a}-\sum_{b=1}^{m} Z_{b}^{a} d z^{b}
$$

so that

$$
\mu_{B}^{a}=\mathbb{D}_{Z}^{B} \mu^{a}=\mathbb{D}_{Z^{b^{1}}} \cdots \mathbb{D}_{Z^{b^{k}}} \mu^{a}, \quad k=\# B .
$$

We refer to [29] for more details.

To concisely express the structure equations of the invariant coframe $\left\{\ldots \sigma^{a} \ldots \mu_{B}^{a} \ldots\right\}$, the vector-valued Maurer-Cartan formal power series $\mu \llbracket H \rrbracket=\left(\mu^{1} \llbracket H \rrbracket, \ldots, \mu^{m} \llbracket H \rrbracket\right)^{T}$ with components

$$
\mu^{a} \llbracket H \rrbracket=\sum_{\# B \geq 0} \frac{1}{B !} \mu_{B}^{a} H^{B}, \quad a=1, \ldots, m,
$$

is introduced. In the above formula, $H=\left(H^{1}, \ldots, H^{m}\right)$ are formal power series parameters while $B !=\widetilde{b}^{1} ! \widetilde{b}^{2} ! \cdots \widetilde{b}^{m}$ ! with $\widetilde{b}^{a}$ standing for the number of occurrences of the integer $1 \leq a \leq m$ in $B$. The structure equations for the Maurer-Cartan forms are then obtained by comparing the coefficients of the various powers of $H$ in the power series identity

$$
d \mu \llbracket H \rrbracket=\nabla \mu \llbracket H \rrbracket \wedge(\mu \llbracket H \rrbracket-d Z),
$$

where $d Z=\left(d Z^{1}, \ldots, d Z^{m}\right)^{T}$ and $\nabla \mu \llbracket H \rrbracket=\left(\partial \mu^{b} \llbracket H \rrbracket / \partial H^{a}\right)$ denotes the $m \times m$ Jacobian matrix obtained by formally differentiating the power series $(2.4)$ with respect to $H=\left(H^{1}, \ldots, H^{m}\right)$. On the other hand, the structure equations for the invariant horizontal one-forms $\sigma=\left(\sigma^{1}, \ldots\right.$, $\left.\sigma^{m}\right)^{T}$ are

$$
d \sigma=\nabla \mu \llbracket 0 \rrbracket \wedge \sigma .
$$

\subsection{Lie pseudo-groups}

Several variants of the technical definition of a Lie pseudo-group exist in the literature, [4, 12, 14, 19, 20, 37]. In the analytic category, Lie pseudo-groups can be defined as follows.

Definition 2.1. A pseudo-group $\mathcal{G} \subset \mathcal{D}$ is called a Lie pseudo-group of order $n_{\star} \geq 1$ if, for all finite $n \geq n_{\star}$ :

- $\mathcal{G}^{(n)} \subset \mathcal{D}^{(n)}$ forms a smooth embedded subbundle,

- the projection $\widetilde{\pi}_{n}^{n+1}: \mathcal{G}^{(n+1)} \rightarrow \mathcal{G}^{(n)}$ is a fibration,

- every local diffeomorphism $\phi \in \mathcal{D}$ satisfying $\mathrm{j}_{n_{\star}} \phi \subset \mathcal{G}^{\left(n_{\star}\right)}$ belongs to $\mathcal{G}$,

- $\mathcal{G}^{(n)}=\operatorname{pr}^{\left(n-n_{\star}\right)} \mathcal{G}^{\left(n_{\star}\right)}$ is obtained by prolongation.

For $n \geq n_{\star}$, Definition 2.1 implies that the pseudo-group jet subbundle $\mathcal{G}^{(n)} \subset \mathcal{D}^{(n)}$ is characterized by a formally integrable system of $n^{\text {th }}$ order differential equations

$$
F^{(n)}\left(z, Z^{(n)}\right)=0
$$

called the $\left(n^{\text {th }}\right.$ order) determining system of $\mathcal{G}^{(n)}$.

At the infinitesimal level, let

$$
\mathbf{v}=\sum_{a=1}^{m} \zeta^{a}(z) \frac{\partial}{\partial z^{a}} \in T M
$$


denote a local vector field on $M$. For $0 \leq n \leq \infty$, let $\mathrm{J}^{n} T M$ denote the bundle of $n^{\text {th }}$ order jets of sections of $T M$ with local coordinates

$$
\left(z, \zeta^{(n)}\right)=\left(\ldots z^{a} \ldots \zeta_{B}^{a} \ldots\right)
$$

where $\zeta_{B}^{a}$ denotes the derivative coordinates of order $0 \leq \# B \leq n$. Let $\mathfrak{g}$ denote the (local) Lie algebra of $\mathcal{G}$ consisting of all local vector fields on $M$ tangent to the pseudo-group orbits. A vector field (2.7) is in $\mathfrak{g}$ if and only if its jets satisfy the $n^{\text {th }}$ order (formally integrable) infinitesimal determining system

$$
L^{(n)}\left(z, \zeta^{(n)}\right)=\sum_{a=1}^{m} \sum_{\# B \leq n} h_{a ; v}^{B}(z) \zeta_{B}^{a}=0, \quad v=1, \ldots, k, \quad n \geq n_{\star},
$$

obtained by linearizing the determining system $(2.6)$ at the identity jet $\mathbb{1}^{(n)}$.

Theorem 2.2. For each $n \geq n_{\star}$, the linear relations among the (restricted) Maurer-Cartan forms $\left.\mu^{(n)}\right|_{\mathcal{G}}$ are obtained by making the formal substitution or "lift" (see (3.6), (3.8) below)

$$
z^{a} \longrightarrow Z^{a}, \quad \zeta_{B}^{a} \longrightarrow \mu_{B}^{a}
$$

in the infinitesimal determining equations (2.8):

$$
L^{(n)}\left(Z, \mu^{(n)}\right)=0 .
$$

Corollary 2.3. The structure equations of a Lie pseudo-group $\mathcal{G}$ are obtained by restricting the diffeomorphism structure equations (2.5) to the solution space of (2.9):

$$
\left.(d \sigma=\nabla \mu \llbracket 0 \rrbracket \wedge \sigma, d \mu \llbracket H \rrbracket=\nabla \mu \llbracket H \rrbracket \wedge(\mu \llbracket H \rrbracket-d Z))\right|_{L^{(\infty)}\left(Z, \mu^{(\infty)}\right)=0} .
$$

Example 2.4. Let $M=\mathrm{J}^{2}\left(\mathbb{R}^{2}, 1\right)$ be the second order jet bundle of curves in the plane with local coordinates

$$
x, \quad u, \quad p=u_{x}, \quad q=u_{x x} .
$$

To illustrate the constructions occurring in this paper we will consider the equivalence problem of second order ordinary differential equations

$$
q=F(x, u, p)
$$

under the Lie pseudo-group of contact transformations

$$
X=\chi(x, u, p), \quad U=\psi(x, u, p), \quad P=\beta(x, u, p), \quad Q=\frac{\beta_{x}+p \beta_{u}+q \beta_{p}}{\chi_{x}+p \chi_{u}+q \chi_{p}}
$$

where the functions $(\chi, \psi, \beta) \in \mathcal{D}\left(\mathbb{R}^{3}\right)$ satisfy the contact conditions

$$
\psi_{p}=\beta \chi_{p}, \quad \beta\left(\chi_{x}+p \chi_{u}\right)=\psi_{x}+p \psi_{u},
$$

and the Lie pseudo-group of point transformations

$$
X=\chi(x, u), \quad U=\psi(x, u), \quad P=\frac{\widehat{D} \psi}{\widehat{D} \chi}, \quad Q=\frac{\widehat{D}^{2} \psi \cdot \widehat{D} \chi-\widehat{D} \psi \cdot \widehat{D}^{2} \chi}{(\widehat{D} \chi)^{3}},
$$

with $(\chi, \psi) \in \mathcal{D}\left(\mathbb{R}^{2}\right)$ and

$$
\widehat{D}=\frac{\partial}{\partial x}+p \frac{\partial}{\partial u}+q \frac{\partial}{\partial p} .
$$


We now compute the low order structure equations for the contact pseudo-group (2.12). Let

$$
\mathbf{v}=\xi(x, u, p, q) \frac{\partial}{\partial x}+\eta(x, u, p, q) \frac{\partial}{\partial u}+\alpha(x, u, p, q) \frac{\partial}{\partial p}+\gamma(x, u, p, q) \frac{\partial}{\partial q}
$$

denote a local vector field on $M=\mathrm{J}^{2}\left(\mathbb{R}^{2}, 1\right)$. The vector field (2.14) is tangent to the orbits of the pseudo-group action (2.12) if and only if

$$
\xi=\xi(x, u, p), \quad \eta=\eta(x, u, p), \quad \alpha=\alpha(x, u, p), \quad \gamma=\widehat{D} \alpha-q \widehat{D} \xi,
$$

and

$$
\eta_{p}=p \xi_{p}, \quad \alpha=\eta_{x}+p\left(\eta_{u}-\xi_{x}\right)+p^{2} \xi_{u}
$$

Hence, the coefficients of the infinitesimal generator (2.14) satisfy the determining system

$$
\xi_{q}=\eta_{q}=\alpha_{q}=0, \quad \eta_{p}=p \xi_{p}, \quad \alpha=\eta_{x}+p\left(\eta_{u}-\xi_{x}\right)+p^{2} \xi_{u}, \quad \gamma=\widehat{D} \alpha-q \widehat{D} \xi .
$$

Under the replacement

$$
\xi_{A} \rightarrow \mu_{A}^{x}, \quad \eta_{A} \rightarrow \mu_{A}^{u}, \quad \alpha_{A} \rightarrow \mu_{A}^{p}, \quad \gamma_{A} \rightarrow \mu_{A}^{q}, \quad(x, u, p, q) \rightarrow(X, U, P, Q),
$$

the infinitesimal determining equations (2.15) yield the linear dependencies

$$
\begin{aligned}
& \mu_{Q}^{x}=\mu_{Q}^{u}=\mu_{Q}^{p}=0, \quad \mu_{P}^{u}=P \mu_{P}^{x}, \quad \mu^{p}=\mu_{X}^{u}+P\left(\mu_{U}^{u}-\mu_{X}^{x}\right)+P^{2} \mu_{U}^{x}, \\
& \mu^{q}=\mu_{X}^{p}+P \mu_{U}^{p}+Q \mu_{P}^{p}-Q\left(\mu_{X}^{x}+P \mu_{U}^{x}+Q \mu_{P}^{x}\right),
\end{aligned}
$$

among the Maurer-Cartan forms of order $\leq 1$. Differentiating $(2.16)$ with respect to $\mathbb{D}_{X}, \mathbb{D}_{Y}$, $\mathbb{D}_{P}, \mathbb{D}_{Q}$ as defined in (2.3), we obtain the linear relations among the higher order Maurer-Cartan forms. It follows that

$$
\mu_{X^{i} U^{j} P^{k}}=\mu_{X^{i} U^{j} P^{k}}^{x}, \quad \nu_{X^{i} U^{j}}=\mu_{X^{i} U^{j}}^{u}, \quad i, j, k \geq 0,
$$

is a basis of Maurer-Cartan forms. Restricting the structure equations of the diffeomorphism pseudo-group $\mathcal{D}\left(\mathbb{R}^{4}\right)$ to $(2.16)$ and its prolongations we obtain the structure equations

$$
\begin{aligned}
d \sigma^{x}= & -d \mu=\mu_{X} \wedge \sigma^{x}+\mu_{U} \wedge \sigma^{u}+\mu_{P} \wedge \sigma^{p} \\
d \sigma^{u}= & -d \nu=\nu_{X} \wedge \sigma^{x}+\nu_{U} \wedge \sigma^{u}+P \mu_{P} \wedge \sigma^{p} \\
d \sigma^{p}= & {\left[\nu_{X X}+P\left(\nu_{U X}-\mu_{X X}\right)+P^{2} \mu_{U X}\right] \wedge \sigma^{x}+\left[\nu_{U X}+P\left(\nu_{U U}-\mu_{U X}\right)+P^{2} \mu_{U U}\right] \wedge \sigma^{u} } \\
& +\left[\nu_{U}-\mu_{X}+P\left(2 \mu_{U}+\mu_{X P}\right)+P\left(2 P \mu_{U P}-\mu_{X P}\right)\right] \wedge \sigma^{p}, \\
d \sigma^{q}= & \mu_{X}^{q} \wedge \sigma^{x}+\mu_{U}^{q} \wedge \sigma^{u}+\mu_{P}^{q} \wedge \sigma^{p}+\mu_{Q}^{q} \wedge \sigma^{q},
\end{aligned}
$$

for the horizontal coframe and the order 0 Maurer-Cartan forms $\mu, \nu$. We do not write the structure equations for the higher order Maurer-Cartan forms as these are not needed subsequently.

For the pseudo-group of point transformations (2.13), it suffices to add the constraints

$$
\xi_{p}=\eta_{p}=0
$$

to $(2.15)$ to obtain the infinitesimal determining equations of its Lie algebra:

$$
\begin{aligned}
& \xi_{p}=\eta_{p}=0, \quad \xi_{q}=\eta_{q}=\alpha_{q}=0, \\
& \alpha=\eta_{x}+p\left(\eta_{u}-\xi_{x}\right)+p^{2} \xi_{u}, \quad \gamma=\widehat{D} \alpha-q\left(\xi_{x}+p \xi_{u}\right) .
\end{aligned}
$$


Taking the lift of (2.18) we obtain the linear relations

$$
\begin{aligned}
& \mu_{P}^{x}=\mu_{P}^{u}=0, \quad \mu_{Q}^{x}=\mu_{Q}^{u}=\mu_{Q}^{p}=0, \quad \mu^{p}=\mu_{X}^{u}+P\left(\mu_{U}^{u}-\mu_{X}^{x}\right)+P^{2} \mu_{U}^{x}, \\
& \mu^{q}=\mu_{X}^{p}+P \mu_{U}^{p}+Q \mu_{P}^{p}-Q\left(\mu_{X}^{x}+P \mu_{U}^{x}\right),
\end{aligned}
$$

among the Maurer-Cartan forms of order $\leq 1$. A basis of Maurer-Cartan forms is thus given by

$$
\mu_{X^{i} U^{j}}=\mu_{X^{i} U^{j}}^{x}, \quad \nu_{X^{i} U^{j}}=\mu_{X^{i} U^{j}}^{u} .
$$

By setting $\mu_{P}=\mu_{P X}=\mu_{P U}=\cdots=0$ in (2.17) we obtain the structure equations for the horizontal coframe $\left\{\sigma^{x}, \sigma^{u}, \sigma^{p}, \sigma^{q}\right\}$. On the other hand, the structure equations for the MaurerCartan forms (2.19) are given by the structure equations of the diffeomorphism pseudo-group $\mathcal{D}\left(\mathbb{R}^{2}\right),[29]$ :

$$
\begin{aligned}
& d \mu=\sigma^{x} \wedge \mu_{X}+\sigma^{u} \wedge \mu_{U}, \\
& d \mu_{X}=\sigma^{x} \wedge \mu_{X X}+\sigma^{u} \wedge \mu_{X U}+\mu_{U} \wedge \nu_{X}, \\
& d \mu_{U}=\sigma^{x} \wedge \mu_{X U}+\sigma^{u} \wedge \mu_{U U}+\mu_{X} \wedge \mu_{U}+\mu_{U} \wedge \nu_{U} \\
& d \nu=\sigma^{x} \wedge \nu_{X}+\sigma^{u} \wedge \nu_{U}, \\
& d \nu_{X}=\sigma^{x} \wedge \nu_{X X}+\sigma^{u} \wedge \nu_{X U}+\nu_{X} \wedge \mu_{X}+\nu_{U} \wedge \nu_{X} \text {, } \\
& d \nu_{U}=\sigma^{x} \wedge \nu_{X U}+\sigma^{u} \wedge \nu_{U U}+\nu_{X} \wedge \mu_{U} \\
& d \nu_{U U}=\sigma^{x} \wedge \nu_{X U U}+\sigma^{u} \wedge \nu_{U U U}+2 \nu_{X U} \wedge \nu_{U}+\nu_{X} \wedge \mu_{U U}+\nu_{U U} \wedge \nu_{U} \text {, } \\
& d \nu_{X U}=\sigma^{x} \wedge \nu_{X X U}+\sigma^{u} \wedge \nu_{X U U}+\nu_{X U} \wedge \mu_{X}+\nu_{X X} \wedge \mu_{U}+\nu_{X} \wedge \mu_{X U}+\nu_{U U} \wedge \nu_{X},
\end{aligned}
$$

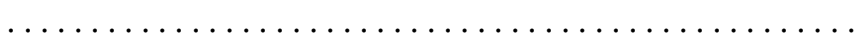

\section{Equivariant moving frames}

As in the previous section, let $\mathcal{G}$ be a Lie pseudo-group acting on an $m$-dimensional manifold $M$. We are now interested in the induced action of $\mathcal{G}$ on $p$-dimensional submanifolds $S \subset M$, where $1 \leq p<m$. For each integer $0 \leq n \leq \infty$, let $\mathrm{J}^{n}=\mathrm{J}^{n}(M, p)$ denote the $n^{\text {th }}$ order submanifold jet bundle, defined as the set of equivalence classes under the equivalence relation of $n^{\text {th }}$ order contact, [26]. For $k \geq n$, we use $\pi_{n}^{k}: \mathrm{J}^{k} \rightarrow \mathrm{J}^{n}$ to denote the canonical projection. We introduce local coordinates $z=(x, u)=\left(x^{1}, \ldots, x^{p}, u^{1}, \ldots, u^{q}\right)$ on $M$ so that submanifolds that are transverse to the vertical fibers $\left\{x=x_{0}\right\}$ are (locally) given as graphs of smooth functions $u=f(x)$. (Submanifolds with vertical tangent spaces are handled by a different choice of local coordinates.) In this adapted system of coordinates, the standard coordinates on $\mathrm{J}^{n}$ are

$$
z^{(n)}=\left(x, u^{(n)}\right)=\left(\ldots x^{i} \ldots u_{J}^{\alpha} \ldots\right),
$$

where $u_{J}^{\alpha}$ denotes the derivative coordinates of order $0 \leq \# J \leq n$.

Let $\mathcal{B}^{(n)} \rightarrow \mathrm{J}^{n}$ denote the $n^{\text {th }}$ order lifted bundle obtained by pulling back $\mathcal{G}^{(n)} \rightarrow M$ via the projection $\pi_{0}^{n}: \mathrm{J}^{n} \rightarrow M$. Local coordinates on $\mathcal{B}^{(n)}$ are given by $\left(z^{(n)}, g^{(n)}\right)$, where the base coordinates $z^{(n)}=\left(x, u^{(n)}\right) \in \mathrm{J}^{n}$ are the submanifold jet coordinates and the fiber coordinates are the pseudo-group parameters $g^{(n)}$. The bundle $\mathcal{B}^{(n)}$ carries the structure of a groupoid with source map $\boldsymbol{\sigma}^{(n)}\left(z^{(n)}, g^{(n)}\right)=z^{(n)}$ and target map $\boldsymbol{\tau}^{(n)}\left(z^{(n)}, g^{(n)}\right)=Z^{(n)}=g^{(n)} \cdot z^{(n)}$ given by the prolonged action. To compute the prolonged action we introduce on $\mathcal{B}^{(\infty)}$ the lifted horizontal coframe

$$
\omega^{i}=d_{H} X^{i}=\sum_{j=1}^{p}\left(D_{x^{j}} X^{i}\right) d x^{j}, \quad i=1, \ldots, p,
$$


where $D_{x^{1}}, \ldots, D_{x^{p}}$ are the total derivative operators. The lifted total derivative operators $D_{X^{1}}, \ldots, D_{X^{p}}$ are then defined by the formula

$$
d_{H} F\left(z^{(n)}\right)=\sum_{i=1}^{p}\left(D_{x^{i}} F\right) d x^{i}=\sum_{i=1}^{p}\left(D_{X^{i}} F\right) \omega^{i} .
$$

More explicitly,

$$
D_{X^{i}}=\sum_{j=1}^{p} \widehat{W}_{i}^{j} D_{x^{j}}, \quad \text { with } \quad\left(\widehat{W}_{i}^{j}\right)=\left(D_{x^{i}} X^{j}\right)^{-1} .
$$

Differentiating the target dependent variables $U^{\alpha}$ with respect to the lifted total derivative operators (3.1) we obtain the explicit expressions for the prolonged action $Z^{(n)}=g^{(n)} \cdot z^{(n)}$ :

$$
X^{i}, \quad U_{J}^{\alpha}=D_{X}^{J} U^{\alpha}
$$

A local diffeomorphism $h \in \mathcal{G}$ acts on $\left\{\left(z^{(n)}, g^{(n)}\right) \in \mathcal{B}^{(n)} \mid \pi_{0}^{n}\left(z^{(n)}\right) \in \operatorname{dom} h\right\}$ by right multiplication:

$$
R_{h}\left(z^{(n)}, g^{(n)}\right)=\left(h^{(n)} \cdot z^{(n)}, g^{(n)} \cdot\left(h^{(n)}\right)^{-1}\right) .
$$

The $\left(n^{\text {th }}\right.$ order) lifted action action (3.3) is given by the concatenation of the prolonged action on submanifold jets with the restriction of the right action $(2.1)$ to $\mathcal{G}^{(n)}$. The expressions (3.2) are invariant under the lifted action (3.3) and these functions are called lifted invariants.

\subsection{Regular submanifold jets}

The existence of a moving frame requires the prolonged pseudo-group action on submanifold jets to be (locally) free and regular, [30].

Definition 3.1. A pseudo-group acts regularly in the neighborhood of a point $z^{(n)} \in \mathrm{J}^{n}$ if the pseudo-group orbits have the same dimension and there are arbitrarily small neighborhoods whose intersection with each orbit is a connected subset thereof.

Definition 3.2. The isotropy subgroup of a submanifold jet $z^{(n)} \in \mathrm{J}^{n}$ is defined as

$$
\mathcal{G}_{z^{(n)}}^{(n)}=\left\{\left.g^{(n)} \in \mathcal{G}^{(n)}\right|_{z}: g^{(n)} \cdot z^{(n)}=z^{(n)}\right\},
$$

where $\pi_{0}^{n}\left(z^{(n)}\right)=z$. The pseudo-group is said to act freely at $z^{(n)}$ if $\mathcal{G}_{z^{(n)}}^{(n)}=\left\{\left.\mathbb{1}^{(n)}\right|_{z}\right\}$. The pseudo-group acts locally freely at $z^{(n)}$ if $\mathcal{G}_{z^{(n)}}^{(n)}$ is discrete.

Definition 3.3. A submanifold jet $z^{(\infty)}$ is said to be regular if there exists a finite $n \geq 1$ such that the pseudo-group $\mathcal{G}$ acts freely at $z^{(n)}=\pi_{n}^{\infty}\left(z^{(\infty)}\right)$. Let $\mathcal{V}^{\infty} \subset \mathrm{J}^{\infty}$ denote the subset of all regular submanifold jets.

Following the foundational papers $[28,30]$ we, for the moment, assume that the pseudogroup $\mathcal{G}$ acts regularly on $\mathcal{V}^{\infty}$ and that this set is a dense open subbundle of $\mathrm{J}^{\infty}$. In Example 3.13 , we will see that, in general, these assumptions need to be relaxed.

Definition 3.4. Let $\mathcal{V}^{n}=\pi_{n}^{\infty}\left(\mathcal{V}^{\infty}\right)$ denote the truncation of the regular submanifold jets to order $n$. A (right) moving frame of order $n$ is a (local) $\mathcal{G}$-equivariant section $\widehat{\rho}^{(n)}: \mathcal{V}^{n} \rightarrow \mathcal{B}^{(n)}$. 
In local coordinates we use the notation

$$
\widehat{\rho}^{(n)}\left(z^{(n)}\right)=\left(z^{(n)}, \rho^{(n)}\left(z^{(n)}\right)\right)
$$

to denote a moving frame. Right equivariance means that

$$
R_{g} \widehat{\rho}^{(n)}\left(z^{(n)}\right)=\widehat{\rho}^{(n)}\left(g^{(n)} \cdot z^{(n)}\right), \quad g \in \mathcal{G}, \quad \text { when defined. }
$$

Theorem 3.5. Suppose $\mathcal{G}$ acts (locally) freely on $\mathcal{V}^{n} \subset \mathrm{J}^{n}$, with its orbits forming a regular foliation, then an $n^{\text {th }}$ order moving frame exists in a neighborhood of every $z^{(n)} \in \mathcal{V}^{n}$.

Once a pseudo-group action is free, a result known as the persistence of freeness, [28, 31], guarantees that the action remains free under prolongation.

Theorem 3.6. If a Lie pseudo-group $\mathcal{G}$ acts (locally) freely at $z^{(n)}$ then it acts (locally) freely at any $z^{(k)} \in \mathrm{J}^{k}, k \geq n$, with $\pi_{n}^{k}\left(z^{(k)}\right)=z^{(n)}$. The minimal $n$ such that the action becomes free is called the order of freeness and is denoted by $n^{\star}$.

An order $n \geq n^{\star}$ moving frame is constructed through a normalization procedure based on the choice of a cross-section $\mathcal{K}^{n} \subset \mathcal{V}^{n}$ to the pseudo-group orbits. The associated (locally defined) right moving frame section $\widehat{\rho}^{(n)}: \mathcal{V}^{n} \rightarrow \mathcal{B}^{(n)}$ is uniquely characterized by the condition that $\boldsymbol{\tau}^{(n)}\left(\widehat{\rho}^{(n)}\left(z^{(n)}\right)\right)=\rho^{(n)}\left(z^{(n)}\right) \cdot z^{(n)} \in \mathcal{K}^{n}$. For simplicity, we assume that $\mathcal{K}^{n}=\left\{z_{i_{1}}=\right.$ $\left.c_{1}, \ldots, z_{i_{n}}=c_{r_{n}}: r_{n}=\left.\operatorname{dim} \mathcal{G}^{(n)}\right|_{z}\right\}$ is a coordinate cross-section. Then, the moving frame $\widehat{\rho}^{(n)}$ is obtained by solving the normalization equations

$$
Z_{i_{1}}\left(x, u^{(n)}, g^{(n)}\right)=c_{1}, \quad \ldots \quad Z_{i_{r_{n}}}\left(x, u^{(n)}, g^{(n)}\right)=c_{r_{n}}
$$

for the pseudo-group parameters $g^{(n)}=\rho^{(n)}\left(x, u^{(n)}\right)$. The invariants appearing on the left-hand side of the normalization equations (3.4) are called phantom invariants. As one increases the order from $n$ to $k>n$, a new cross-section must be selected. We require that these crosssections be compatible in the sense that $\pi_{n}^{k}\left(\mathcal{K}^{k}\right)=\mathcal{K}^{n}$ for all $k>n$. This in turn, implies the compatibility of the moving frames: $\pi_{n}^{k}\left(\widehat{\rho}^{(k)}\left(z^{(k)}\right)\right)=\widehat{\rho}^{(n)}\left(\pi_{n}^{k}\left(z^{(k)}\right)\right)$. A compatible sequence of moving frames is simply called a moving frame and is denoted by $\widehat{\rho}: \mathcal{V}^{\infty} \rightarrow \mathcal{B}^{(\infty)}$. Finally, we require the compatible cross-sections to be of minimal order, [28]. Intuitively, this is equivalent to requiring that the pseudo-group parameters be normalized as soon as possible during the normalization procedure.

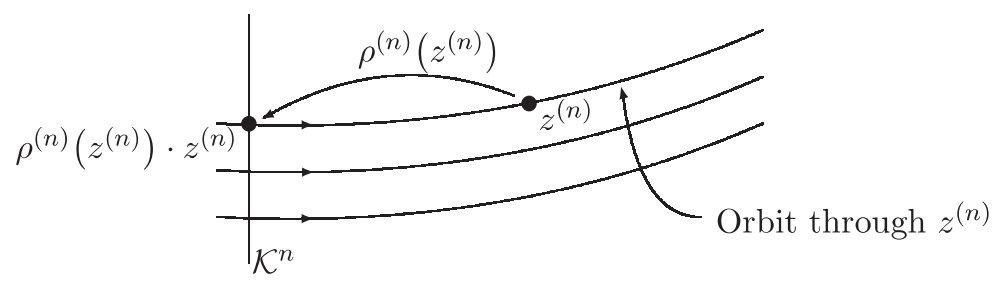

Figure 1. Moving frame $\widehat{\rho}^{(n)}\left(z^{(n)}\right)=\left(z^{(n)}, \rho^{(n)}\left(z^{(n)}\right)\right)$.

We now introduce the invariantization map associated with a moving frame. First, we note that the space of differential forms on $\mathcal{B}^{(\infty)}$ splits into

$$
\mathbf{\Omega}^{*}=\bigoplus_{k, l} \boldsymbol{\Omega}^{k, l}=\bigoplus_{i, j, l} \boldsymbol{\Omega}^{i, j, l}
$$


where $l$ indicates the number of Maurer-Cartan forms (2.2), $k=i+j$ the number of jet forms, with $i$ indicating the number of horizontal forms $d x^{i}$ and $j$ the number of basic submanifold jet contact forms

$$
\theta_{J}^{\alpha}=d u_{J}^{\alpha}-\sum_{i=1}^{p} u_{J, i}^{\alpha} d x^{i}, \quad \alpha=1, \ldots, q, \quad \# J \geq 0 .
$$

Next, let

$$
\boldsymbol{\Omega}_{J}^{*}=\bigoplus_{k} \boldsymbol{\Omega}^{k, 0}=\bigoplus_{i, j} \boldsymbol{\Omega}^{i, j, 0}
$$

denote the subspace of jet forms consisting of those differential forms containing no MaurerCartan forms. Then, we introduce the projection $\pi_{J}: \Omega^{*} \rightarrow \Omega_{J}^{*}$ which takes a differential form $\Omega$ on $\mathcal{B}^{(\infty)}$ to its jet component $\pi_{J}(\Omega)$ obtained by annihilating the Maurer-Cartan forms in $\Omega$.

Definition 3.7. Let $\Omega$ be a differential form defined on $J^{\infty}$. Its lift is the invariant jet form

$$
\lambda(\Omega)=\pi_{J}\left[\left(\tau^{(\infty)}\right)^{*}(\Omega)\right]
$$

defined on the lifted bundle $\mathcal{B}^{(\infty)}$.

In particular, setting $\Omega$ in (3.5) to be each of the submanifold jet coordinates $x^{i}, u_{J}^{\alpha}$, the lift map (3.5) coincides with the prolonged action (3.2):

$$
\lambda\left(x^{i}\right)=X^{i}, \quad \lambda\left(u_{J}^{\alpha}\right)=U_{J}^{\alpha} .
$$

Also, we note that the lift of the horizontal forms $d x^{1}, \ldots, d x^{p}$

$$
\lambda\left(d x^{i}\right)=\sum_{i=1}^{p}\left(D_{x^{j}} X^{i}\right) d x^{j}+\sum_{\alpha=1}^{q} X_{u^{\alpha}}^{i} \theta^{\alpha}=\omega^{i}+\sum_{\alpha=1}^{q} X_{u^{\alpha}}^{i} \theta^{\alpha}
$$

are invariant horizontal forms if and only if the pseudo-group action is projectable meaning that $X_{u^{\alpha}}^{i}=0$. On the other hand, the lift of a contact form is always a contact form. In the following, we ignore contact forms and introduce the equivalence relation $\equiv$ to indicate equality modulo contact forms.

Definition 3.8. Let $\widehat{\rho}: \mathcal{V}^{\infty} \rightarrow \mathcal{B}^{(\infty)}$ be a moving frame. If $\Omega$ is a differential form on $\mathcal{V}^{\infty}$, then its invariantization is the invariant differential form

$$
\iota(\Omega)=\widehat{\rho}^{*}[\boldsymbol{\lambda}(\Omega)] .
$$

In the following, to lighten the notation, we will usually omit writing moving frame pull-backs:

$$
\begin{aligned}
& \omega^{j}=\widehat{\rho}^{*}\left(\omega^{j}\right) \equiv \widehat{\rho}^{*}\left[\boldsymbol{\lambda}\left(d x^{j}\right)\right]=\iota\left(d x^{j}\right), \\
& \left(X, U^{(\infty)}\right)=\widehat{\rho}^{*}\left(X, U^{(\infty)}\right)=\widehat{\rho}^{*}\left[\boldsymbol{\lambda}\left(x, u^{(\infty)}\right)\right]=\iota\left(x, u^{(\infty)}\right) .
\end{aligned}
$$

Proposition 3.9. The normalized differential invariants

$$
X^{i}=\iota\left(x^{i}\right), \quad U_{J}^{\alpha}=\iota\left(u_{J}^{\alpha}\right),
$$

contain a complete set of functionally independent differential invariants.

One of the most important results in the theory of equivariant moving frames is the universal recurrence formula for lifted/invariantized differential forms, [30]. To write down the formula we must extend the lift map (3.5) to vector field jet coordinates. 
Definition 3.10. The lift of a vector jet coordinate $\zeta_{B}^{a}$ is defined to be the Maurer-Cartan form $\mu_{B}^{a}$ :

$$
\lambda\left(\zeta_{B}^{a}\right)=\mu_{B}^{a}, \quad \text { for } \quad a=1, \ldots, m, \quad \# B \geq 0 .
$$

More generally, the lift of any finite linear combination of vector field jet coordinates is

$$
\boldsymbol{\lambda}\left[\sum_{a=1}^{m} \sum_{\# B \geq 0} P_{a}^{B}\left(x, u^{(n)}\right) \zeta_{B}^{a}\right]=\sum_{a=1}^{m} \sum_{\# B \geq 0} P_{a}^{B}\left(X, U^{(n)}\right) \mu_{B}^{a} .
$$

Recall that if

$$
\mathbf{v}=\sum_{i=1}^{p} \xi^{i}(x, u) \frac{\partial}{\partial x^{i}}+\sum_{\alpha=1}^{q} \phi_{\alpha}(x, u) \frac{\partial}{\partial u^{\alpha}} \in \mathfrak{g}
$$

is an infinitesimal generator of the pseudo-group action, then its prolongation is the vector field

$$
\mathbf{v}^{(\infty)}=\sum_{i=1}^{p} \xi^{i}(x, u) \frac{\partial}{\partial x^{i}}+\sum_{\alpha=1}^{q} \sum_{\# J \geq 0} \phi_{\alpha}^{J} \frac{\partial}{\partial u_{J}^{\alpha}} \in \mathfrak{g}^{(\infty)},
$$

where

$$
\phi_{\alpha}^{J}=D_{J} Q^{\alpha}+\sum_{i=1}^{p} \xi^{i} u_{J, i}^{\alpha}, \quad \text { and } \quad Q^{\alpha}=\phi_{\alpha}-\sum_{i=1}^{p} \xi^{i} u_{i}^{\alpha}
$$

are the characteristic components of the vector field (3.9). Then, for $\left.z^{(\infty)} \in \mathrm{J}^{\infty}\right|_{z}$ the prolongation formula (3.10) defines the prolongation map

$$
\mathbf{p}=\mathbf{p}_{z^{(\infty)}}^{(\infty)}:\left.\left.\quad \mathrm{J}^{\infty} T M\right|_{z} \rightarrow T \mathrm{~J}^{\infty}\right|_{z^{(\infty)}}, \quad \mathbf{p}_{z^{(\infty)}}^{(\infty)}\left(\left.\mathrm{j}_{\infty} \mathbf{v}\right|_{z}\right)=\left.\mathbf{v}^{(\infty)}\right|_{z^{(\infty)}}
$$

Theorem 3.11. Let $\Omega$ be a differential form on $\mathrm{J}^{\infty}$. Then

$$
d[\boldsymbol{\lambda}(\Omega)]=\boldsymbol{\lambda}\left[d \Omega+\mathbf{v}^{(\infty)}(\Omega)\right], \quad \text { where } \quad \mathbf{v}^{(\infty)} \in \mathfrak{g}^{(\infty)}
$$

and $\mathbf{v}^{(\infty)}(\Omega)$ denotes the Lie derivative of $\Omega$ along $\mathbf{v}^{(\infty)}$.

We refer to [30] for a proof of (3.12). In particular, the identity (3.12) applies to the lifted differential invariants $X^{i}, U_{J}^{\alpha}$ :

$$
\begin{aligned}
& d X^{i} \equiv \omega^{i}+\mu^{i}, i=1, \ldots, p, \\
& d U_{J}^{\alpha} \equiv \sum_{j=1}^{p} U_{J, j}^{\alpha} \omega^{j}+\lambda\left(\phi_{\alpha}^{J}\right), \quad \alpha=1, \ldots, q, \quad \# J \geq 0 .
\end{aligned}
$$

Corollary 3.12. Let $\widehat{\rho}: \mathcal{V}^{\infty} \rightarrow \mathcal{B}^{(\infty)}$ be a moving frame and $\Omega$ a differential form on $\mathcal{V}^{\infty}$, then

$$
d[\iota(\Omega)]=\iota\left[d \Omega+\mathbf{v}^{(\infty)}(\Omega)\right], \quad \text { where } \quad \mathbf{v}^{(\infty)} \in \mathfrak{g}^{(\infty)} .
$$

Of particular interest to us is when $\Omega$ is one of the submanifold jet coordinate functions $x^{i}, u_{J}^{\alpha}$. Equation (3.14) then produces the recurrence relations

$$
\mathcal{D}_{j} X^{i}=\delta_{j}^{i}+N_{j}^{i}, \quad \mathcal{D}_{j} U_{J}^{\alpha}=U_{J, j}^{\alpha}+M_{J, j}^{\alpha},
$$


where $N_{j}^{i}$ and $M_{J, j}^{\alpha}$ are correction terms and

$$
\mathcal{D}_{k}=\sum_{j=1}^{p} \widehat{\rho}^{*}\left(W_{k}^{j}\right) D_{x^{j}}, \quad k=1, \ldots, p,
$$

are invariant total derivative operators obtained by normalizing the lifted total derivative operators (3.1).

The commutation relations among the invariant total derivative operators $\mathcal{D}_{1}, \ldots, \mathcal{D}_{p}$ can be deduced from the universal recurrence relation (3.14). By setting $\Omega=d x^{i}$ in (3.14) we obtain the equations

$$
d \omega^{i} \equiv-\sum_{1 \leq j<k \leq p} Y_{j k}^{i} \omega^{j} \wedge \omega^{k}, \quad i=1, \ldots, p .
$$

Since the operators $\mathcal{D}_{i}$ are dual to the contact invariant horizontal forms $\omega^{i}$, it follows that

$$
\left[\mathcal{D}_{i}, \mathcal{D}_{j}\right]=\sum_{k=1}^{p} Y_{i j}^{k} \mathcal{D}_{k}, \quad 1 \leq i, j \leq p,
$$

and the invariant functions $Y_{j k}^{i}$ are called commutator invariants, [28]. An important feature of the recurrence formula (3.14) (or (3.15)) is that the coordinate expressions for the invariants $\left(X, U^{(\infty)}\right)$, the contact invariant horizontal 1-forms $\omega^{i}$, the Maurer-Cartan forms $\mu_{B}^{a}$ and the moving frame $\widehat{\rho}$ are not required to compute these equations. One only needs to know the cross-section $\mathcal{K}^{\infty} \subset \mathcal{V}^{\infty}$ defining $\widehat{\rho}$ and the expression of $\mathbf{v}^{(\infty)} \in \mathfrak{g}^{(\infty)}$. The key observation is that the pulled-back Maurer-Cartan forms $\widehat{\rho}^{*}\left(\mu_{B}^{a}\right)$ can be obtained from the phantom invariant recurrence relations. We refer the reader to $[6,30,31]$ for concrete examples of the moving frame implementation.

Example 3.13. As mentioned at the beginning of this section, the theory introduced above assumes the Lie pseudo-group action to be free and regular on a dense open subset $\mathcal{V}^{\infty} \subset \mathrm{J}^{\infty}$. Using the local equivalence problem of second order ordinary differential equations under the pseudo-group of point transformations (2.13), we now show that, in general, these assumptions should be relaxed. Working symbolically, we use the recurrence relations (3.13) to find disjoint sets of regular submanifold jets each admitting their own moving frame.

The first step consists of determining the "universal normalizations" which hold for any second order ordinary differential equation. Beginning with the order 0 recurrence relations

$$
\begin{aligned}
& d X \equiv \omega^{x}+\mu \\
& d U \equiv \omega^{u}+\nu \\
& d P \equiv \omega^{p}+\nu_{X}+P\left(2 \nu_{Y}-\mu_{X}\right)-P^{2} \mu_{U} \\
& d Q \equiv Q_{P} \omega^{p}+Q_{U} \omega^{u}+Q_{X} \omega^{x}+\nu_{X X}+Q\left(\nu_{U}-2 \mu_{X}\right)+P\left(2 \nu_{X U}-\mu_{X X}\right) \\
&-3 P Q \mu_{U}+P^{2}\left(\nu_{U U}-2 \mu_{X U}\right)-P^{3} \mu_{U U},
\end{aligned}
$$

the lone appearance of the linearly independent Maurer-Cartan forms $\mu, \nu, \nu_{X}, \nu_{X X}$ in the group differential component of the recurrence relations (3.18) implies that we can normalize the lifted invariants

$$
X=U=P=Q=0
$$

to zero, independently of the differential equations. Substituting (3.19) into (3.18) we obtain a system of equations that can be solved for the (partially) normalized Maurer-Cartan forms

$$
\mu \equiv-\omega^{x}, \quad \nu \equiv-\omega^{u}, \quad \nu_{X} \equiv-\omega^{p}, \quad \nu_{X X} \equiv-\left(Q_{P} \omega^{p}+Q_{U} \omega^{u}+Q_{X} \omega^{x}\right) .
$$


Continuing the normalization procedure, order by order, we come to the conclusion that it is always possible to normalize the lifted invariants

$$
\begin{aligned}
& X=U=P=0, \\
& Q_{U^{j} X^{k}}=Q_{P U^{j} X^{k}}=Q_{P^{2} U^{j}}=Q_{P^{2} U^{j} X}=Q_{P^{3} U^{j}}=Q_{P^{3} U^{j} X}=0, \quad j, k \geq 0,
\end{aligned}
$$

to zero (see [24, Appendix B] for more details). The normalizations (3.20) lead to the normalization of all the Maurer-Cartan forms (2.19) except for

$$
\mu_{X}, \quad \mu_{U}, \quad \nu_{U}, \quad \nu_{U U}, \quad \nu_{X U}
$$

To proceed further, the value of the remaining (partially normalized) lifted invariants

$$
Q_{P^{k+4} U^{j} X^{i}}, \quad Q_{P^{3} U^{j} X^{i+2}}, \quad Q_{P^{2} U^{j} X^{i+2}}, \quad i, j, k \geq 0,
$$

must be carefully analyzed. More explicitly, the invariants $(3.22)$ of order $\leq 6$ are

$$
\begin{array}{ll}
n=4: & Q_{P^{4}}, Q_{P^{2} X^{2}}, \\
n=5: & Q_{P^{5}}, Q_{P^{4} U}, Q_{P^{4} X}, Q_{P^{3} X^{2}}, Q_{P^{2} U X^{2}}, Q_{P^{2} X^{3}}, \\
n=6: & Q_{P^{6}}, Q_{P^{5} U}, Q_{P^{5} X}, Q_{P^{4} U^{2}}, Q_{P^{4} U X}, Q_{P^{4} X^{2}}, Q_{P^{3} U X^{2}}, \\
& Q_{P^{3} X^{3}}, Q_{P^{2} U^{2} X^{2}}, Q_{P^{2} U X^{3}}, Q_{P^{2} X^{4}} .
\end{array}
$$

Writing the recurrence relations for the invariants (3.23) of order $\leq 5$, taking into account the normalizations (3.20), we obtain

$$
\begin{aligned}
& d Q_{P^{4}} \equiv Q_{P^{5}} \omega^{p}+Q_{P^{4} U} \omega^{u}+Q_{P^{4} X} \omega^{x}+Q_{P^{4}}\left(2 \mu_{X}-3 \nu_{U}\right),
\end{aligned}
$$

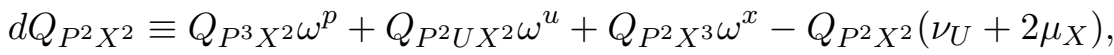

$$
\begin{aligned}
& d Q_{P^{5}} \equiv Q_{P^{6}} \omega^{p}+Q_{P^{5} U} \omega^{u}+Q_{P^{5} X} \omega^{x}+5 Q_{P^{4}} \mu_{U}+Q_{P^{5}}\left(3 \mu_{X}-4 \nu_{U}\right), \\
& d Q_{P^{4} X} \equiv\left(Q_{P^{5} X}+Q_{P^{4} U}\right) \omega^{p}+Q_{P^{4} U X} \omega^{u}+Q_{P^{4} X^{2}} \omega^{x}+Q_{P^{4} \nu_{U X}}+Q_{P^{4} X}\left(\mu_{X}-3 \nu_{U}\right) \text {, } \\
& d Q_{P^{4} U} \equiv Q_{P^{5} U} \omega^{p}+Q_{P^{4} U^{2}} \omega^{u}+Q_{P^{4} U X} \omega^{x}-2 Q_{P^{4}} \nu_{U U}-Q_{P^{5}} \nu_{U X}-Q_{P^{4} X} \mu_{U} \\
& +Q_{P^{4} U}\left(2 \mu_{X}-4 \nu_{U}\right) \text {, } \\
& d Q_{P^{3} X^{2}} \equiv Q_{P^{4} X^{2}} \omega^{p}+Q_{P^{3} U X^{2}} \omega^{u}+\left(Q_{P^{3} X^{3}}-2 Q_{P^{2} U X^{2}}\right) \omega^{x}-Q_{P^{2} X^{2}} \mu_{U} \\
& -Q_{P^{3} X^{2}}\left(2 \nu_{U}+\mu_{X}\right) \text {, } \\
& d Q_{P^{2} U X^{2}} \equiv Q_{P^{3} U X^{2}} \omega^{p}+Q_{P^{2} U^{2} X^{2}} \omega^{u}+Q_{P^{2} U X^{3}} \omega^{x}-2 Q_{P^{2} X^{2}} \nu_{U U}-Q_{P^{3} X^{2}} \nu_{U X} \\
& -Q_{P^{2} X^{3}} \mu_{U}-2 Q_{P^{2} U X^{2}}\left(\nu_{U}+\mu_{X}\right) \text {, } \\
& d Q_{P^{2} X^{3}}=\left(Q_{P^{3} X^{3}}-Q_{P^{2} U X^{2}}\right) \omega^{p}+Q_{P^{2} U X^{3} \omega^{u}}+Q_{P^{2} X^{4}} \omega^{x}-5 Q_{P^{2} X^{2}} \nu_{U X} \\
& -Q_{P^{2} X^{3}}\left(\nu_{U}+3 \mu_{X}\right) \text {. }
\end{aligned}
$$

At this juncture, the normalization procedure splits into different branches depending on the value of the fourth-order lifted invariants

$$
Q_{P^{4}}=\frac{\chi_{x}^{2}}{\psi_{u}^{3}} q_{p p p p}, \quad Q_{P^{2} X^{2}}=\frac{\widehat{D}^{2}\left(q_{p p}\right)-4 \widehat{D}\left(q_{u p}\right)-q_{p} \widehat{D}\left(q_{p p}\right)+6 q_{u u}-3 q_{u} q_{p p}+4 q_{p} q_{u p}}{\psi_{u} \chi_{x}^{2}}
$$

obtained by implementing the moving frame construction. There are 4 branches to consider ${ }^{1}$
I) $Q_{P^{4}} \not \equiv 0$ and $Q_{P^{2} X^{2}} \not \equiv 0$,
III) $Q_{P^{4}} \not \equiv 0$ and $Q_{P^{2} X^{2}} \equiv 0$,
II) $Q_{P^{4}} \equiv 0$ and $Q_{P^{2} X^{2}} \not \equiv 0$,
IV) $Q_{P^{4}} \equiv 0$ and $Q_{P^{2} X^{2}} \equiv 0$.

\footnotetext{
${ }^{1}$ To distinguish between lifted invariants that are set equal to a constant by normalization from those that are identically constant, we use the notation $=$ and $\equiv$ respectively.
} 
In Case IV) the pseudo-group action is not free and we differ this branch of the equivalence problem to the next section. For the other three cases, let

$$
\mathcal{K}^{\infty}=\left\{x=u=p=q_{u^{j} x^{k}}=q_{p u^{j} x^{k}}=q_{p^{2} u^{j}}=q_{p^{2} u^{j} x}=q_{p^{3} u^{j}}=q_{p^{3} u^{j} x}=0 \mid j, k \geq 0\right\}
$$

denote the "intermediate cross-section" corresponding to the universal normalizations (3.20). In Case I), the action is free on the subbundle of regular submanifold jets

$$
\mathcal{V}_{1}^{\infty}=\left\{z^{(\infty)} \in \mathrm{J}^{\infty} \mid Q_{P^{4}} \not \equiv 0, Q_{P^{2} X^{2}} \not \equiv 0\right\}
$$

From the recurrence relations (3.24), we find that a cross-section is given by

$$
\mathcal{K}_{1}^{\infty}=\mathcal{K}^{\infty} \cup\left\{q_{p^{4}}=q_{p^{2} x^{2}}=1, q_{p^{5}}=q_{p^{4} u}=q_{p^{4} x}=0\right\} \subset \mathcal{V}_{1}^{\infty}
$$

leading to a moving frame $\widehat{\rho}_{1}: \mathcal{V}_{1}^{\infty} \rightarrow \mathcal{B}^{(\infty)}$. In Case II), it can be shown, see [24] for more details, that when $Q_{P^{2} X^{2}} \not \equiv 0$ the (partially normalized) lifted invariants $Q_{P^{3} X^{3}}$ and $Q_{P^{2} X^{4}}$ cannot be simultaneously equal to zero. There are then 2 subbundles of regular submanifold jets

$$
\begin{aligned}
& \mathcal{V}_{2}^{\infty}=\left\{z^{(\infty)} \in \mathrm{J}^{\infty} \mid Q_{P^{2} X^{2}} \not \equiv 0, D_{J} Q_{P^{4}} \equiv 0, Q_{P^{2} X^{4}} \not \equiv 0\right\}, \\
& \mathcal{V}_{3}^{\infty}=\left\{z^{(\infty)} \in \mathrm{J}^{\infty} \mid Q_{P^{2} X^{2}} \not \equiv 0, D_{J} Q_{P^{4}} \equiv 0, Q_{P^{3} X^{3}} \not \equiv 0\right\} .
\end{aligned}
$$

In the above formula, the total derivative operator $D_{J}=D_{j^{1}} \cdots D_{j^{k}}$ in the variables $x^{1}=x$, $x^{2}=u, x^{3}=p$ ranges over the multi-indices of length $\# J \geq 0$. Admissible cross-sections are given by

$$
\begin{aligned}
& \mathcal{K}_{2}^{\infty}=\mathcal{K}^{\infty} \cup\left\{q_{p^{2} x^{2}}=1, q_{p^{3} x^{2}}=q_{p^{2} u x^{2}}=q_{p^{2} x^{3}}=0, q_{p^{2} x^{4}}=1\right\} \subset \mathcal{V}_{2}^{\infty}, \\
& \mathcal{K}_{3}^{\infty}=\mathcal{K}^{\infty} \cup\left\{q_{p^{2} x^{2}}=1, q_{p^{3} x^{2}}=q_{p^{2} u x^{2}}=q_{p^{2} x^{3}}=0, q_{p^{3} x^{3}}=1\right\} \subset \mathcal{V}_{3}^{\infty}
\end{aligned}
$$

Similarly, in Case III) the non-degeneracy condition $Q_{P^{4}} \not \equiv 0$ implies that $Q_{P^{6}}$ and $Q_{P^{5} X}$ cannot both be equal to zero so that on

$$
\begin{aligned}
& \mathcal{V}_{4}^{\infty}=\left\{z^{(\infty)} \in \mathrm{J}^{\infty} \mid D_{J} Q_{P^{2} X^{2}} \equiv 0, Q_{P^{4}} \not \equiv 0, Q_{P^{6}} \not \equiv 0\right\}, \\
& \mathcal{V}_{5}^{\infty}=\left\{z^{(\infty)} \in \mathrm{J}^{\infty} \mid D_{J} Q_{P^{2} X^{2}} \equiv 0, Q_{P^{4}} \not \equiv 0, Q_{P^{5} X} \not \equiv 0\right\},
\end{aligned}
$$

moving frames can be constructed. On $\mathcal{V}_{4}^{\infty}$ and $\mathcal{V}_{5}^{\infty}$ possible cross-sections are given by

$$
\begin{aligned}
& \mathcal{K}_{4}^{\infty}=\mathcal{K}^{\infty} \cup\left\{q_{p^{4}}=1, q_{p^{5}}=q_{p^{4} u}=q_{p^{4} x}=0, q_{p^{6}}=1\right\} \subset \mathcal{V}_{4}^{\infty}, \\
& \mathcal{K}_{5}^{\infty}=\mathcal{K}^{\infty} \cup\left\{q_{p^{4}}=1, q_{p^{5}}=q_{p^{4} u}=q_{p^{4} x}=0, q_{p^{5} x}=1\right\} \subset \mathcal{V}_{5}^{\infty},
\end{aligned}
$$

respectively.

As illustrated by the above computations, the assumption that $\mathcal{V}^{\infty}$ is a dense open subset of $\mathrm{J}^{\infty}$ on which the pseudo-group acts regularly (and freely) is too restrictive. The generic Case I) corresponding the set of regular submanifold jets $\mathcal{V}_{1}^{\infty}$ is the only branch of the equivalence problem satisfying this assumption. To encompass the other cases, we observe that, apart from a finite number of non-degeneracy conditions, $\mathcal{V}_{2}^{\infty}, \ldots, \mathcal{V}_{5}^{\infty}$ are characterized by $\mathcal{G}$-invariant systems of differential equations which we formalize in the following definition.

Definition 3.14. Let $\mathcal{G}$ be a Lie pseudo-group acting on $\mathrm{J}^{\infty}$. A $\mathcal{G}$-invariant subbundle $S^{\infty} \subset \mathrm{J}^{\infty}$ is said to be regular of order $\bar{n} \geq 1$ if, for all finite $n \geq \bar{n}$ :

- $S^{n}=\pi_{n}^{\infty}\left(S^{\infty}\right) \subset \mathrm{J}^{n}$ forms a smooth embedded subbundle, 
- the projection $\pi_{n}^{n+1}: S^{n+1} \rightarrow S^{n}$ is a fibration,

- $S^{n}=\operatorname{pr}^{(n-\bar{n})} S^{\bar{n}}$ is obtained by prolongation,

- $S^{n}$ is $\mathcal{G}$-invariant.

In light of the above comments, we allow the set of regular submanifold jets to be the finite union

$$
\mathcal{V}^{\infty}=\bigcup_{i=1}^{k} \mathcal{V}_{i}^{\infty}
$$

of dense open subsets of $\mathcal{G}$-invariant regular subbundles of $\mathrm{J}^{\infty}$. In other words, the subbundles $\mathcal{V}_{i}^{\infty}$ are $\mathcal{G}$-invariant Zariski open subsets of $\mathrm{J}^{\infty}$. Definition 3.14 implies that for each subbundle $\mathcal{V}_{i}^{\infty}$ there exists $n_{i} \geq 1$ such that for $n \geq n_{i}$ the subbundle $\mathcal{V}_{i}^{n}=\pi_{n}^{\infty}\left(\mathcal{V}_{i}^{\infty}\right) \subset \mathrm{J}^{n}$ is characterized by a $\mathcal{G}$-invariant system of formally integrable differential equations

$$
E_{i}^{(n)}\left(x, u^{(n)}\right)=0
$$

plus, possibly, a finite number of $\mathcal{G}$-invariant non-degeneracy conditions.

Example 3.15. The determining equations (3.26) and the non-degeneracy conditions naturally occur as one tries to normalize the parameters of an equivalence pseudo-group in the moving frame algorithm. In Example 3.13, the determining system for $\mathcal{V}_{1}^{\infty}$ is trivial while the nondegeneracy conditions are given by

$$
Q_{P^{4}} \not \equiv 0, \quad Q_{P^{2} X^{2}} \not \equiv 0 .
$$

For $\mathcal{V}_{2}^{\infty}$, the order of regularity is $n_{2}=4$, and for $n \geq n_{2}$ the determining system of $\mathcal{V}_{2}^{n}$ is

$$
D_{J}\left(Q_{P^{4}}\right) \equiv 0, \quad 0 \leq \# J \leq n-n_{2},
$$

to which we add the non-degeneracy conditions $Q_{P^{2} X^{2}} \not \equiv 0, Q_{P^{2} X^{4}} \not \equiv 0$.

Remark 3.16. While the pseudo-group action will, in general, not be regular on the whole set of regular submanifold jets $\mathcal{V}^{\infty}$, in order to construct a moving frame on each invariant subbundle $\mathcal{V}_{i}^{\infty}$, we require the restriction of pseudo-group action to $\mathcal{V}_{i}^{\infty}$ to be regular in the subset topology.

Assuming the action is free and regular on each invariant regular subbundle $\mathcal{V}_{i}^{\infty}$, we can construct a moving frame $\widehat{\rho}_{i}: \mathcal{V}_{i}^{\infty} \rightarrow \mathcal{B}_{i}^{(\infty)}\left(\mathcal{B}_{i}^{(\infty)}=\mathcal{B}^{(\infty)} \mid \mathcal{V}_{i}^{\infty}\right)$ by choosing a cross-section $\mathcal{K}_{i}^{\infty} \subset \mathcal{V}_{i}^{\infty}$ Each moving frame will have its own order of freeness $n_{i}^{\star} \geq n_{i}$ and on each subbundle $\mathcal{V}_{i}^{\infty}$ the recurrence relations (3.15) completely determine the algebra of differential invariants.

\subsection{Singular submanifold jets}

We now would like to extend the moving frame method to submanifold jets $z^{(\infty)}$ where a pseudogroup does not act freely.

Example 3.17. Any pseudo-group satisfying

$$
r_{n}=\left.\operatorname{dim} \mathcal{G}^{(n)}\right|_{z}>\left.\operatorname{dim} \mathrm{J}^{n}\right|_{z}=q\left(\begin{array}{c}
p+n \\
p
\end{array}\right) \quad \text { for all } n \geq 1
$$

cannot act freely. Indeed, the inequality (3.27) implies that for all $n \geq 1$ the isotropy group $\mathcal{G}_{z^{(n)}}^{(n)}$ is non-trivial since the dimension of the pseudo-group jet $\left.\mathcal{G}^{(n)}\right|_{z}$ is larger than the dimension of the jet space $\mathrm{J}^{n}$ on which it acts. The contact pseudo-group (2.12) is an example of such pseudo-group. 
Example 3.18. A second example is given by Case IV) of Example 3.13. When $Q_{P^{4}} \equiv$ $Q_{P^{2} X^{2}} \equiv 0$ it follows from the recurrence relations (3.24) that the fifth and sixth order (partially normalized) lifted invariants (3.23) are identically equal to zero. This combined with the higher order recurrence relations then implies that all higher order invariants (3.22) are also equal to zero. Hence, in Case IV) there are no further lifted invariants available to normalize the Maurer-Cartan forms (3.21). Said differently, on the subbundle

$$
\mathcal{S}^{\infty}=\left\{z^{(\infty)} \in \mathrm{J}^{\infty} \mid D_{J} Q_{P^{4}} \equiv D_{J} Q_{P^{2} X^{2}} \equiv 0, \# J \geq 0\right\} .
$$

the pseudo-group parameters

$$
\chi_{x}, \quad \chi_{u}, \quad \psi_{u}, \quad \psi_{u u}, \quad \psi_{x u}
$$

cannot be normalized and these pseudo-group jets parametrize the 5-dimensional isotropy group $\mathcal{G}_{z^{(\infty)}}^{(\infty)}$ of a submanifold jet $z^{(\infty)} \in \mathcal{S}^{\infty}$.

Definition 3.19. A submanifold jet $z^{(\infty)} \in \mathrm{J}^{\infty}$ is said to be singular if its isotropy group is non-trivial:

$$
\mathcal{G}_{z^{(\infty)}}^{(\infty)}=\left\{\left.g^{(\infty)} \in \mathcal{G}^{(\infty)}\right|_{z}: g^{(\infty)} \cdot z^{(\infty)}=z^{(\infty)}\right\} \neq\left\{\left.\mathbb{1}^{(\infty)}\right|_{z}\right\}
$$

The set of singular submanifold jets is denoted by $\mathcal{S}^{\infty}$.

As with regular submanifold jets, we allow the set of singular submanifold jets to be a finite union of open dense subsets of $\mathcal{G}$-invariant regular subbundles

$$
\mathcal{S}^{\infty}=\bigcup_{i=1}^{\ell} \mathcal{S}_{i}^{\infty}
$$

so that for $n \geq n_{i} \geq 1$, the subbundle $\mathcal{S}_{i}^{n}=\pi_{n}^{\infty}\left(\mathcal{S}_{i}^{\infty}\right)$ is characterized by a formally integrable system of $\mathcal{G}$-invariant differential equations (with possibly finitely many invariant non-degeneracy conditions).

Remark 3.20. Though the pseudo-group action on $\mathcal{S}_{i}^{\infty}$ is not free, we still require the restriction of the action to $\mathcal{S}_{i}^{\infty}$ to be regular in the subset topology.

Let $z^{(\infty)} \in \mathcal{S}_{i}^{\infty}$ be a fixed submanifold jet and $z^{(n)}=\pi_{n}^{\infty}\left(z^{(\infty)}\right) \in \mathcal{S}_{i}^{n}$ its $n^{\text {th }}$ order truncation. Then $\mathcal{G}_{z^{(\infty)}}^{(\infty)}$ is the projective limit

$$
\mathcal{G}_{z^{(\infty)}}^{(\infty)}=\lim _{\longleftarrow} \mathcal{G}_{z^{(n)}}^{(n)} \text {. }
$$

Apart from the order 0 constraint $g \cdot z=z$, we observe that the isotropy requirement $g^{(n)} \cdot z^{(n)}=$ $z^{(n)}$ gives a system of differential constraints for the pseudo-group parameters $g^{(n)} \in \mathcal{G}_{z^{(n)}}^{(n)}$, and the prolongation of this system is given by $g^{(n+1)} \cdot z^{(n+1)}=z^{(n+1)}$. To see this, consider an analytic submanifold $(x, u(x))$ with $\left(x, \mathrm{j}_{\infty} u(x)\right)=z^{(\infty)}$. Then, given

$$
X=\chi(x, u), U=\psi(x, u) \in \mathcal{G}_{z},
$$

the isotropy condition $g^{(n)} \cdot z^{(n)}=z^{(n)}$ is obtained by differentiating

$$
u(\chi(x, u(x)))=\psi(x, u(x))
$$

with respect to the independent variables $x=\left(x^{1}, \ldots, x^{p}\right)$. By Cartan-Kuranishi's prolongation theorem, [2, 35], we conclude that, generically, there exists $n_{i}^{\star} \geq n_{i}$ such that the system of differential equations $g^{\left(n^{\star}\right)} \cdot z^{\left(n^{\star}\right)}=z^{\left(n^{\star}\right)}$ is formally integrable (and eventually involutive for some $n \geq n_{i}^{\star}$ ). In the following, $n_{i}^{\star}$ is assumed to be independent of the submanifold jet $z^{(\infty)} \in \mathcal{S}_{i}^{\infty}$ and $n_{i}^{\star}$ is called the order of partial freeness. For $n \geq n_{i}^{\star}$ the pseudo-group $\mathcal{G}$ is said to act partially freely on $\mathcal{S}_{i}^{n}$. 
Remark 3.21. If $z^{(\infty)} \in \mathcal{V}_{i}^{\infty}$ is a regular submanifold jet, the order of partial freeness defined above corresponds to the usual order of freeness. In this case the only solution to $g^{(n)} \cdot z^{(n)}=z^{(n)}$ is $\mathcal{G}_{z^{(n)}}^{(n)}=\left\{\left.\mathbb{1}^{(n)}\right|_{z}\right\}$ for $n \geq n_{i}^{\star}$.

Example 3.22. To illustrate the above discussion we consider the Lie pseudo-group

$$
X=f(x), \quad Y=e(x, y)=f^{\prime}(x) y+g(x), \quad U=u+\frac{e_{x}(x, y)}{f^{\prime}(x)}
$$

acting on graphs of functions $(x, y, u(x, y))$. This is one of the main examples used in [28, 30] to illustrate the moving frame method. The pseudo-group (3.28) acts locally freely on the set of regular submanifold jets $\mathcal{V}_{-}^{\infty}=\mathrm{J}^{\infty} \cap\left\{u_{y y}<0\right\}$ and $\mathcal{V}_{+}^{\infty}=\mathrm{J}^{\infty} \cap\left\{u_{y y}>0\right\}$. On the other hand, when restricted to the jet space of linear functions $u(x, y)=a(x) y+b(x)$ given by

$$
\mathcal{S}^{\infty}=\mathrm{J}^{\infty} \cap\left\{u_{x^{i} y^{j+2}}=0: i, j \geq 0\right\},
$$

the pseudo-group action is not free. To obtain the determining equations of the isotropy pseudogroup at a submanifold jet $z^{(\infty)}=\left(x, y, u^{(\infty)}\right) \in \mathcal{S}^{\infty}$ we differentiate the equality

$$
u(f(x), e(x, y))=u(x, y)+\frac{e_{x}(x, y)}{f_{x}(x)}
$$

with respect to $x$ and $y$. Up to order 2 , taking into account that $u_{y y}=0$ on $\mathcal{S}^{\infty}$, we obtain the constraints

$$
\begin{aligned}
& f=x, \quad e=y, \quad u_{x} f_{x}+u_{y} e_{x}=u_{x}+\frac{e_{x x}}{f_{x}}-\frac{e_{x} f_{x x}}{f_{x}^{2}}, \quad u_{y} f_{x}=u_{y}+\frac{f_{x x}}{f_{x}}, \\
& u_{x x} f_{x}+u_{x} f_{x x}+u_{x y} f_{x} e_{x}+u_{y} e_{x x}=u_{x x}+\frac{e_{x x x}}{f_{x}}-2 \frac{e_{x x} f_{x x}}{f_{x}^{2}}-\frac{e_{x} f_{x x x}}{f_{x}^{2}}+2 \frac{e_{x} f_{x x}^{2}}{f_{x}^{3}}, \\
& u_{x y} f_{x}^{2}+u_{y} f_{x x}=u_{x y}+\frac{f_{x x x}}{f_{x}}-\frac{f_{x x}^{2}}{f_{x}^{2}}
\end{aligned}
$$

on the jets of $f(x)$ and $e(x, y)$. Solving the equations (3.30) (and their prolongations) for the pseudo-group parameters we conclude that $f_{x}$ parametrizes the isotropy group $\mathcal{G}_{z^{(\infty)}}^{(\infty)}$.

\subsubsection{Partial moving frames}

Though it is not possible to construct a moving frame on $\mathcal{S}_{i}^{\infty}$ as in Section 3.1, it is nevertheless possible to introduce the notion of a partial moving frame. For $n_{i}^{\star} \leq n \leq \infty$, where $n_{i}^{\star}$ is the order of partial freeness, we introduce the $n^{\text {th }}$ order prolonged bundle

$$
\mathcal{P}_{i}^{(n)}=\left\{\left(z^{(n)}, g^{(n)}\right): z^{(n)} \in \mathcal{S}_{i}^{n} \text { and } g^{(n)} \in \mathcal{G}_{z^{(n)}}^{(n)}\right\} .
$$

A local diffeomorphism $h \in \mathcal{G}$ acts on the set

$$
\left\{\left(z^{(n)}, g^{(n)}\right) \in \mathcal{P}_{i}^{(n)} \mid \pi_{0}^{n}\left(z^{(n)}\right) \in \operatorname{dom} h\right\}
$$

by

$$
h^{(n)} \cdot\left(z^{(n)}, g^{(n)}\right)=\left(h^{(n)} \cdot z^{(n)}, K_{h^{(n)}}\left(g^{(n)}\right)\right),
$$

where $K_{h^{(n)}}\left(g^{(n)}\right)=h^{(n)} \cdot g^{(n)} \cdot\left(h^{-1}\right)^{(n)}$ is the conjugation action. 
Definition 3.23. For $n \geq n_{i}^{\star}$, an $n^{\text {th }}$ order (right) partial moving frame on $\mathcal{S}_{i}^{n}$ is a $\mathcal{G}$-equivariant bundle map

$$
\widehat{\rho}_{i}^{(n)}: \mathcal{P}_{i}^{(n)} \rightarrow \mathcal{B}_{i}^{(n)}, \quad \text { where } \quad \mathcal{B}_{i}^{(n)}=\left.\mathcal{B}^{(n)}\right|_{\mathcal{S}_{i}^{n}} .
$$

In terms of the action $(3.31)$, right $\mathcal{G}$-equivariance means that

$$
R_{h} \widehat{\rho}_{i}^{(n)}\left(z^{(n)}, g^{(n)}\right)=\widehat{\rho}_{i}^{(n)}\left(h^{(n)} \cdot\left(z^{(n)}, g^{(n)}\right)\right) .
$$

A partial moving frame is constructed by following the algorithm of Section 3.1. Namely, a partial moving frame of order $n \geq n_{i}^{\star}$ is obtained by choosing a minimal cross-section $\mathcal{K}_{i}^{n} \subset \mathcal{S}_{i}^{n}$ and solving the normalization equations (3.4). By the implicit function theorem, the normalization equations can be solved near the identity jet and the solution will depend on the submanifold jet $z^{(n)}$ and the isotropy group parameters $g^{(n)} \in \mathcal{G}_{z^{(n)}}^{(n)}$. See Fig. 2 for a suggestive illustration of a partial moving frame.

Theorem 3.24. Let $n \geq n_{i}^{\star}$ so that $\mathcal{G}$ acts partially freely on $\mathcal{S}_{i}^{n} \subset \mathrm{J}^{n}$ with its orbits forming a regular foliation. Then an $n^{\text {th }}$ order partial moving frame exists in a neighborhood of $z^{(n)} \in \mathcal{S}_{i}^{n}$.

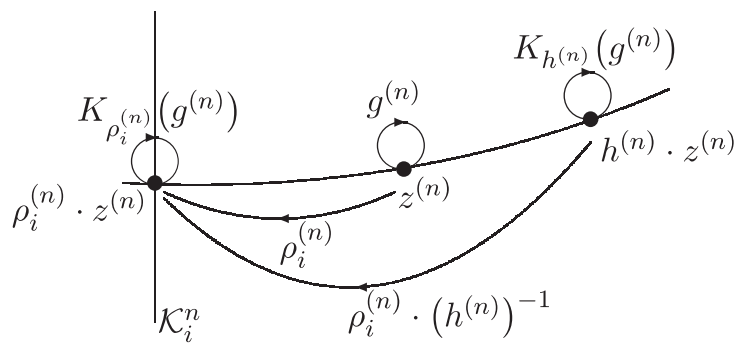

Figure 2. Partial moving frame $\widehat{\rho}_{i}^{(n)}\left(z^{(n)}, g^{(n)}\right)=\left(z^{(n)}, \rho_{i}^{(n)}\left(z^{(n)}, g^{(n)}\right)\right)$, with $g^{(n)} \in \mathcal{G}_{z^{(n)}}^{(n)}$.

A partial moving frame $\widehat{\rho}_{i}: \mathcal{P}_{i}^{(\infty)} \rightarrow \mathcal{B}_{i}^{(\infty)}$ is constructed by choosing a series of compatible cross-sections $\mathcal{K}_{i}^{n} \subset \mathcal{S}_{i}^{n}$ just as in the regular case. The definition of the invariantization map (3.7) and the recurrence formula (3.14) still hold for partial moving frames with the understanding that these formulas are now defined on the prolonged bundle $\mathcal{P}_{i}^{(\infty)}$. We note that none of the normalized differential invariants $\iota\left(z^{(\infty)}\right)=\iota\left(x, u^{(\infty)}\right)$ can depend on the isotropy group parameters $g^{(\infty)} \in \mathcal{G}_{z^{(\infty)}}^{(\infty)}$. On the other hand, the invariantization of the jet forms $d x^{i}, \theta_{J}^{\alpha}$ may involve the isotropy group parameters.

Remark 3.25. The concept of partial moving frame defined above is similar to the recent notion of partial moving frame introduced in [32]. Indeed, given a cross-section $\mathcal{K}_{i}^{\infty} \subset \mathcal{S}_{i}^{\infty}$ defining a partial moving frame $\widehat{\rho}_{i}$, its inverse image $\left(\boldsymbol{\tau}^{(\infty)}\right)^{-1}\left(\mathcal{K}_{i}^{\infty}\right)$ under the restricted target map $\boldsymbol{\tau}^{(\infty)}: \mathcal{B}_{i}^{(\infty)} \rightarrow \mathcal{S}_{i}^{\infty}$ happens to be equal to the image of the partial moving frame $\widehat{\rho}_{i}\left(\mathcal{P}_{i}^{(\infty)}\right)=$ $\left(\boldsymbol{\tau}^{(\infty)}\right)^{-1}\left(\mathcal{K}_{i}^{\infty}\right)$.

Example 3.26. Continuing Example 3.22, and referring to $[28,30]$ for all the formulas, the prolonged bundle over the singular submanifold jets (3.29) is given by

$$
\mathcal{P}^{(\infty)}=\left\{\left(x, y, u^{(\infty)}, f_{x}\right): u_{x^{i} y^{j+2}}=0\right\} .
$$

Up to second order, the expressions for the prolonged action are

$$
U_{X}=\frac{u_{x}}{f_{x}}+\frac{e_{x x}-e_{x} u_{y}}{f_{x}^{2}}-2 \frac{f_{x x} e_{x}}{f_{x}^{3}}, \quad U_{Y}=\frac{u_{y}}{f_{x}}+\frac{f_{x x}}{f_{x}^{2}},
$$




$$
\begin{aligned}
& U_{X X}=\frac{u_{x x}}{f_{x}^{2}} \frac{e_{x x x}-e_{x x} u_{y}-2 e_{x} u_{x y}-f_{x x} u_{x}}{f_{x}^{3}}+\frac{3 e_{x} f_{x x} u_{y}-4 e_{x x} f_{x x}-3 e_{x} f_{x x x}}{f_{x}^{4}}+8 \frac{e_{x} f_{x x}^{2}}{f_{x}^{5}}, \\
& U_{X Y}=\frac{u_{x y}}{f_{x}^{2}}+\frac{f_{x x x}-f_{x x} u_{y}}{f_{x}^{3}}-2 \frac{f_{x x}^{2}}{f_{x}^{4}}, \quad U_{Y Y}=0 .
\end{aligned}
$$

We note that $U_{Y Y} \equiv 0$ when $u_{y y} \equiv 0$ and more generally $U_{X^{i} Y^{j+2}} \equiv 0, i, j \geq 0$. A cross-section to the pseudo-group orbit is given by

$$
\mathcal{K}^{\infty}=\left\{x=y=u_{x^{i}}=u_{x^{i} y}=0, i \geq 0\right\} \subset \mathcal{S}^{\infty} .
$$

Solving the normalization equations $X=Y=U_{X^{i}}=U_{X^{i} Y}=0$, we obtain the partial moving frame

$$
f=e=0, \quad e_{x}=-u f_{x}, \quad f_{x x}=-u_{y} f_{x}, \quad e_{x x}=\left(u u_{y}-u_{x}\right) f_{x}, \quad \ldots
$$

Since the action is transitive on (3.29) there are no differential invariants. The (partially) invariantized horizontal coframe is

$$
\omega^{x}=\iota(d x)=f_{x} d x, \quad \omega^{y}=f_{x}(d y-u d x),
$$

and their structure equations are

$$
d \omega^{x} \equiv \mu_{X} \wedge \omega^{x}, \quad d \omega^{y} \equiv \mu_{X} \wedge \omega^{y}, \quad d \mu_{X} \equiv 0,
$$

where

$$
\mu_{X}=\widehat{\rho}^{*}\left(\mu_{X}\right)=\frac{d f_{x}}{f_{x}}+u_{y} d x
$$

is the only unnormalized Maurer-Cartan form reflecting the fact that the pseudo-group jet $f_{x}$ parametrizes the isotropy group of a submanifold jet $z^{(\infty)} \in \mathcal{S}^{\infty}$. The structure equations (3.32) are isomorphic to the Maurer-Cartan structure equations of a 3-dimensional Lie group indicating that the surface $(x, y, a(x) y+b(x))$ is invariant under a 3-dimensional group of transformations lying inside the pseudo-group (3.28).

\section{Local equivalence}

In this section we review the solution to the local equivalence problem of submanifolds using the equivariant moving frame machinery. We then explain how Cartan's approach based on the theory of $G$-structures is related to the equivariant method.

\subsection{Equivalence of submanifolds}

Given two $p$-dimensional submanifolds $S$ and $\bar{S}$ in $M$, the local equivalence problem consists of determining whether there exists or not a local diffeomorphism $g \in \mathcal{G}$ such that $g \cdot S=\bar{S}$ locally. In accordance with Cartan's general philosophy, the solution to this problem is determined by the invariants of $\mathcal{G}$. Let $\mathcal{K}_{i}^{\infty}$ be a cross-section defining a (partial) moving frame $\widehat{\rho}_{i}$. Since the normalized invariants $\left(X, U^{(\infty)}\right)=\iota\left(x, u^{(\infty)}\right)$ give a local coordinate system on $\mathcal{K}_{i}^{\infty}$, two submanifolds $S$ and $\bar{S}$ are locally equivalent if and only if both submanifolds lie in the domain of definition of the same (partial) moving frame and their "projections" $\iota\left(\mathrm{j}_{\infty} S\right), \iota\left(\mathrm{j}_{\infty} \bar{S}\right)$ onto $\mathcal{K}_{i}^{\infty}$ overlap (see Fig. 3).

Definition 4.1. Let $\mathcal{D}_{1}, \ldots, \mathcal{D}_{p}$ be the invariant total derivative operators (3.16). A set of differential invariants $\left\{I_{\kappa}\right\}$ is said to be a generating set for the algebra of differential invariants if any invariant can be locally expressed as a function of the invariants $I_{\kappa}$ and their invariant derivatives $\mathcal{D}_{J} I_{\kappa}=\mathcal{D}_{j_{1}} \mathcal{D}_{j_{2}} \cdots \mathcal{D}_{j_{k}} I_{\kappa}$. 

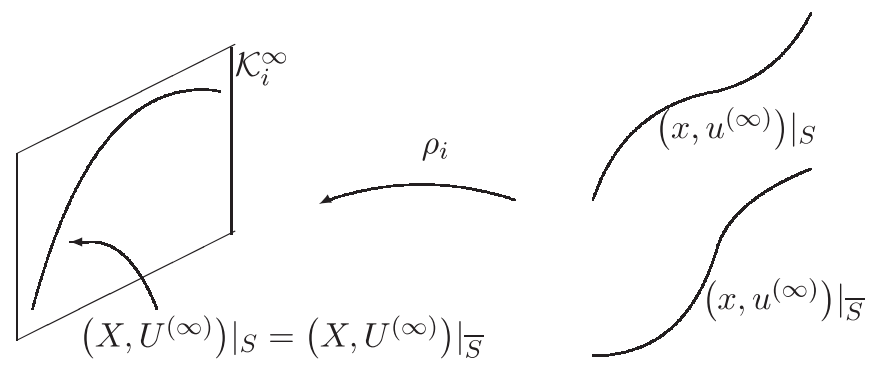

Figure 3. Signature of two equivalent submanifolds.

First proved by Lie, [22, p. 760], for finite-dimensional Lie group actions and then extended to infinite-dimensional Lie pseudo-groups by Tresse, [41], the fundamental basis theorem (also known as the Lie-Tresse theorem) guarantees that, under appropriate technical hypotheses, the algebra of differential invariants is generated by a finite number of differential invariants and $p$ invariant total derivative operators. For Lie group actions, recent proofs based on the equivariant moving frame method can be found in [9, 13]. For infinite-dimensional Lie pseudogroups, a proof also exists, [28], but it is much more technical. It requires the introduction of two modules associated with the prolonged pseudo-group action, and assumes the action to be free and regular on a dense open subset of $\mathrm{J}^{\infty}$. This more abstruse part of the theory is discussed in Section 5 where we explain how to modify the algebraic constructions introduced in [28] to cover Lie pseudo-groups acting regularly and freely on invariant regular subbundles of $\mathrm{J}^{\infty}$. Further modifications will also allow us to deal with Lie pseudo-groups acting regularly and nonfreely on invariant regular subbundles of $\mathrm{J}^{\infty}$. Other proofs based on Spencer cohomology, [19], Weyl algebras, [25], and homological methods, [18], are also available. A global version of the Theorem for transitive algebraic pseudo-group actions was recently proved by Kruglikov and Lychagin in [17].

Given a Lie pseudo-group $\mathcal{G}$ with regular submanifold jets $\mathcal{V}^{\infty}=\cup \mathcal{V}_{i}^{\infty}$ and singular submanifold jets $\mathcal{S}^{\infty}=\cup \mathcal{S}_{i}^{\infty}$, the fundamental basis theorem applies to each subbundle $\mathcal{V}_{i}^{\infty}$ and $\mathcal{S}_{i}^{\infty}$. Let $\left\{I_{\kappa}\right\}$ be a generating set on $\mathcal{V}_{i}^{\infty}\left(\right.$ or $\left.\mathcal{S}_{i}^{\infty}\right)$. Then, the normalized invariants $\left(X, U^{(\infty)}\right)=$ $\iota\left(x, u^{(\infty)}\right)$ can be expressed in terms of these invariants and their invariant derivatives

$$
X^{i}=\iota\left(x^{i}\right)=F^{i}\left(\ldots I_{\kappa} \ldots \mathcal{D}_{K} I_{\kappa} \ldots\right), \quad U_{J}^{\alpha}=\iota\left(u_{J}^{\alpha}\right)=F_{J}^{\alpha}\left(\ldots I_{\kappa} \ldots \mathcal{D}_{K} I_{\kappa} \ldots\right) .
$$

Hence, if $\mathrm{j}_{\infty} S, \mathrm{j}_{\infty} \bar{S} \subset \mathcal{V}_{i}^{\infty}$ (or $\mathcal{S}_{i}^{\infty}$ ), it follows that their invariantization $\iota\left(\mathrm{j}_{\infty} S\right), \iota\left(\mathrm{j}_{\infty} \bar{S}\right)$ is locally prescribed by the generating invariants and their invariant derivatives.

Definition 4.2. Let $\mathcal{V}^{\infty}=\cup \mathcal{V}_{i}^{\infty}$ and $\mathcal{S}^{\infty}=\cup \mathcal{S}_{i}^{\infty}$ denote the sets of regular and singular submanifold jets. A submanifold $S \subset M$ is said to be regular (or singular) if $\mathrm{j}_{\infty} S \subset \mathcal{V}_{i}^{\infty}$ (or $\left.\mathrm{j}_{\infty} S \subset \mathcal{S}_{i}^{\infty}\right)$ for some $i$.

Remark 4.3. Globally, it is possible that $\mathrm{j}_{\infty} S$ does not lie in a unique subbundle $\mathcal{V}_{i}^{\infty}$ or $\mathcal{S}_{i}^{\infty}$. If so, the submanifold $S$ should be restricted to an open subset where the containment holds.

Definition 4.4. Let $S$ be a regular (or singular) submanifold with $\mathrm{j}_{\infty} S \subset \mathcal{V}_{i}^{\infty}$ (or $\mathrm{j}_{\infty} S \subset \mathcal{S}_{i}^{\infty}$ ). Let $\widehat{\rho}_{i}$ be a moving frame (or a partial moving frame) and $\left\{I_{1}, \ldots, I_{\ell}\right\}$ a generating set for the algebra of differential invariants. The $n^{\text {th }}$ order signature space $\mathbb{K}^{(n)}$ is the Euclidean space of dimension $\ell\left(1+p+p^{2}+\cdots+p^{n}\right)$ coordinatized by $w^{(n)}=\left(\ldots, w_{\kappa ; J}, \ldots\right)$, where $(\kappa ; J)=$ $\left(\kappa, j^{1}, \ldots, j^{r}\right)$ with $1 \leq \kappa \leq \ell$ and $\left(j^{1}, \ldots, j^{r}\right)$ ranging through all unordered multi-index with $1 \leq j^{i} \leq p$ and $0 \leq r \leq n$. The $n^{\text {th }}$ order signature map associated with $\widehat{\rho}_{i}$ is the map $\mathbf{I}_{S}^{(n)}: S \rightarrow \mathbb{K}^{(n)}$ whose components are

$$
w_{\kappa ; J}=\left.\left(\mathcal{D}_{J} I_{\kappa}\right)\right|_{j_{\infty} S}, \quad \kappa=1, \ldots, \ell, \quad \# J \leq n .
$$


Remark 4.5. In Definition 4.4 the multi-index $J$ is not assumed to be symmetric as the invariant total derivative operators $\mathcal{D}_{j}$ do not commute in general. In applications we can reduce the dimension of the $n^{\text {th }}$ order signature space $\mathbb{K}^{(n)}$ by ordering as many multi-indices $J$ as possible using the commutation relations (3.17) and expressing the commutator invariants $Y_{i j}^{k}$ in terms of the generating invariants and their invariant derivatives:

$$
\left[\mathcal{D}_{i}, \mathcal{D}_{j}\right]=\sum_{k=1}^{p} Y_{i j}^{k}\left(\ldots I_{\kappa} \ldots \mathcal{D}_{K} I_{\kappa} \ldots\right) \mathcal{D}_{k}, \quad i, j=1, \ldots, p .
$$

Definition 4.6. The rank of the signature $\operatorname{map} \mathbf{I}_{S}^{(n)}$ at a point $z \in S$ is the dimension of the space spanned by the differentials

$$
\left.d\left[\left.\left(\mathcal{D}_{J} I_{\kappa}\right)\right|_{\mathrm{j}_{\infty} S}\right]\right|_{z}, \quad 1 \leq \kappa \leq \ell, \quad 0 \leq \# J \leq n .
$$

The signature map $\mathbf{I}_{S}^{(n)}$ is regular if its rank is constant on $S$.

Definition 4.7. Let $S$ be a regular (or singular) submanifold with $\mathrm{j}_{\infty} S \subset \mathcal{V}_{i}^{\infty}$ (or $\mathrm{j}_{\infty} S \subset \mathcal{S}_{i}^{\infty}$ ). The restricted moving frame (or partial moving frame) $\widehat{\rho}_{S}=\left.\widehat{\rho}_{i}\right|_{\mathrm{j}_{\infty} S}$ is said to be fully regular if for each $n \geq 0$ the signature $\operatorname{map} \mathbf{I}_{S}^{(n)}: S \rightarrow \mathbb{K}^{(n)}$ is regular.

Definition 4.8. Let $\widehat{\rho}_{S}$ be fully regular. The image of the $n^{\text {th }}$ order signature map $\mathbf{I}_{S}^{(n)}$

$$
\mathfrak{S}^{(n)}\left(\widehat{\rho}_{S}\right)=\left\{\mathbf{I}_{S}^{(n)}(z): z \in S\right\} \subset \mathbb{K}^{(n)}
$$

is called the $n^{\text {th }}$ order signature manifold.

Proposition 4.9. Let $\widehat{\rho}_{S}$ be fully regular, and let $\varrho_{n}$ denote the rank of the $n^{\text {th }}$ order signature $\operatorname{map} \mathbf{I}_{S}^{(n)}$. Then

$$
0 \leq \varrho_{0}<\varrho_{1}<\cdots<\varrho_{s}=\varrho_{s+1}=\cdots=r \leq p=\operatorname{dim} S .
$$

The stabilizing rank $r$ is called the rank of $\widehat{\rho}_{S}$, and the smallest $s$ for which $\varrho_{s}=\varrho_{s+1}=r$ is called the order of $\hat{\rho}_{S}$.

Depending if the two submanifolds $S, \bar{S} \subset M$ are regular or singular we are now ready to state the solution to the local equivalence problem.

Theorem 4.10. Let $\mathcal{G}$ be a Lie pseudo-group acting regularly and freely on $\mathcal{V}_{i}^{\infty} \subset \mathrm{J}^{\infty}(M, p)$, and $\widehat{\rho}_{i}: \mathcal{V}_{i}^{\infty} \rightarrow \mathcal{B}_{i}^{(\infty)}$ a moving frame. If $S, \bar{S} \subset M$ are two regular $p$-dimensional submanifolds with $\mathrm{j}_{\infty} S, \mathrm{j}_{\infty} \bar{S} \subset \mathcal{V}_{i}^{\infty}$, then there exists a local diffeomorphism $g \in \mathcal{G}$ mapping $S$ onto $\bar{S}$ locally if and only if $\widehat{\rho}_{S}$ and $\widehat{\rho}_{\bar{S}}$ are fully regular, have the same order $s=\bar{s}$, and the $(s+1)^{s t}$ order signature manifolds $\mathfrak{S}^{(s+1)}\left(\widehat{\rho}_{S}\right), \mathfrak{S}^{(s+1)}\left(\widehat{\rho}_{\bar{S}}\right)$ overlap. Moreover, if $z \in S$ and $\bar{z} \in \bar{S}$ are mapped to the same point

$$
\mathbf{I}_{S}^{(s+1)}(z)=\mathbf{I}_{\bar{S}}^{(s+1)}(\bar{z}) \in \mathfrak{S}^{(s+1)}\left(\widehat{\rho}_{S}\right) \cap \mathfrak{S}^{(s+1)}\left(\widehat{\rho}_{\bar{S}}\right)
$$

on the overlap of the two signature manifolds, then there exists a unique local diffeomorphism $g \in \mathcal{G}$ mapping $z$ to $\bar{z}=g \cdot z$.

Proof. Let $J_{1}, \ldots, J_{r}$ be a set of invariants parametrizing the $s^{\text {th }}$ order signature manifold $\mathfrak{S}^{(s)}\left(\widehat{\rho}_{S}\right)$. By assumption, there exist signature functions $F_{K ; \kappa}\left(w_{1}, \ldots, w_{r}\right)$, such that

$$
\mathcal{D}_{K} I_{\kappa}=F_{K ; \kappa}\left(J_{1}, \ldots, J_{r}\right), \quad \kappa=1, \ldots, \ell, \quad \# K \leq s .
$$


Furthermore, the invariants $J_{1}, \ldots, J_{r}$ also parametrize the $(s+1)^{\text {th }}$ signature manifold $\mathfrak{S}^{(s+1)}\left(\widehat{\rho}_{S}\right)$. Hence, there exist signature functions $\widetilde{F}_{i ; v}\left(w_{1}, \ldots, w_{r}\right)$ such that

$$
\mathcal{D}_{i} J_{v}=\widetilde{F}_{i ; v}\left(J_{1}, \ldots, J_{r}\right), \quad v=1, \ldots, r, \quad i=1, \ldots, p .
$$

By the chain rule

$$
\mathcal{D}_{i}\left(\mathcal{D}_{K} I_{\kappa}\right)=\sum_{v=1}^{r} \frac{\partial F_{K ; \kappa}}{\partial J_{v}}\left(J_{1}, \ldots, J_{r}\right) \cdot \widetilde{F}_{i ; v}\left(J_{1}, \ldots, J_{r}\right),
$$

and we conclude that once $\mathfrak{S}^{(s+1)}\left(\widehat{\rho}_{S}\right)$ is known, the signature manifold $\mathfrak{S}^{(s+k)}\left(\widehat{\rho}_{S}\right)$ for $k \geq 2$ is obtained by invariant differentiation.

Using the assumption that the signature manifolds $\mathfrak{S}^{(s+1)}\left(\widehat{\rho}_{S}\right)$ and $\mathfrak{S}^{(s+1)}\left(\widehat{\rho}_{\bar{S}}\right)$ overlap, it follows that the invariants $\left\{I_{1}, \ldots, I_{\ell}\right\}$ generating the algebra of differential invariants and their invariant derivatives are locally equal when restricted to $S$ and $\bar{S}$. By (4.1), the restriction of the the normalized invariants $\left.X^{i}\right|_{S}=\left.X^{i}\right|_{S}$ and $\left.U_{K}^{\alpha}\right|_{S}=\left.U_{K}^{\alpha}\right|_{S}$ are also equal. Hence, given $z \in S$ and $\bar{z} \in \bar{S}$ for which $\mathbf{I}_{S}^{(s+1)}(z)=\mathbf{I}_{\bar{S}}^{(s+1)}(\bar{z})$ we conclude that $\iota\left(\left.\mathrm{j}_{\infty} S\right|_{z}\right)=\iota\left(\left.\mathrm{j}_{\infty} \bar{S}\right|_{\bar{z}}\right)$. Now, let $h$, $\bar{h} \in \mathcal{G}$ such that

$$
\left.h^{(\infty)}\right|_{z}=\rho_{i}\left(\left.\mathrm{j}_{\infty} S\right|_{z}\right) \quad \text { and }\left.\quad \bar{h}^{(\infty)}\right|_{\bar{z}}=\rho_{i}\left(\left.\mathrm{j}_{\infty} \bar{S}\right|_{\bar{z}}\right) .
$$

Since $\widetilde{\boldsymbol{\sigma}}^{(\infty)}\left(\left(\left.\bar{h}^{(\infty)}\right|_{\bar{z}}\right)^{-1}\right)=\iota\left(\left.\mathrm{j}_{\infty} \bar{S}\right|_{\bar{z}}\right)=\iota\left(\left.\mathrm{j}_{\infty} S\right|_{z}\right)=\widetilde{\boldsymbol{\tau}}^{(\infty)}\left(\left.h^{(\infty)}\right|_{z}\right)$, the map $g=\bar{h}^{-1} \circ h \in \mathcal{G}$ is (locally) well defined and by construction $\left.g^{(\infty)} \cdot \mathrm{j}_{\infty} S\right|_{z}=\left.\mathrm{j}_{\infty} \bar{S}\right|_{\bar{z}}$. In the analytic category, this implies that $g \cdot S=\bar{S}$ locally, and in particular $g \cdot z=\bar{z}$.

Theorem 4.11. Let $\mathcal{G}$ be a Lie pseudo-group acting regularly on $\mathcal{S}_{i}^{\infty} \subset \mathrm{J}^{\infty}(M, p)$ and $\widehat{\rho}_{i}: \mathcal{P}_{i}^{(\infty)} \rightarrow$ $\mathcal{B}_{i}^{(\infty)}$ a partial moving frame. If $S, \bar{S} \subset M$ are two $p$-dimensional singular submanifolds with $\mathrm{j}_{\infty} S, \mathrm{j}_{\infty} \bar{S} \subset \mathcal{S}_{i}^{\infty}$, then there exists a local diffeomorphism $g \in \mathcal{G}$ sending $S$ onto $\bar{S}$ locally if and only if $\widehat{\rho}_{S}$ and $\widehat{\rho}_{\bar{S}}$ are fully regular, have the same order $s=\bar{s}$, and the $(s+1)^{\text {st }}$ order signature manifolds $\mathfrak{S}^{(s+1)}\left(\widehat{\rho}_{S}\right)$, $\mathfrak{S}^{(s+1)}\left(\widehat{\rho}_{\bar{S}}\right)$ overlap. Moreover, if $z \in S$ and $\bar{z} \in \bar{S}$ are mapped to the same point

$$
\mathbf{I}_{S}^{(s+1)}(z)=\mathbf{I}_{\bar{S}}^{(s+1)}(\bar{z}) \in \mathfrak{S}^{(s+1)}\left(\widehat{\rho}_{S}\right) \cap \mathfrak{S}^{(s+1)}\left(\widehat{\rho}_{\bar{S}}\right)
$$

on the overlap of the two signature manifolds, then there is a family of local equivalence maps sending $z$ to $\bar{z}$. Any two equivalence maps $g, \bar{g}$ are related by

$$
\bar{g}=\bar{h} \circ g \circ h, \quad \text { with }\left.\quad \bar{h}^{(\infty)}\right|_{\bar{z}} \in \mathcal{G}_{\left.\mathrm{j}_{\infty} \bar{S}\right|_{\bar{z}}}^{(\infty)} \quad \text { and }\left.\quad h^{(\infty)}\right|_{z} \in \mathcal{G}_{\left.\mathrm{j}_{\infty} S\right|_{z}}^{(\infty)} .
$$

Proof. The argument is identical to the proof of Theorem 4.10. The only difference is that the local diffeomorphism $g \in \mathcal{G}$ mapping $S$ onto $\bar{S}$ in the neighborhoods of $z \in S$ and $\bar{z} \in \bar{S}$ is not uniquely defined. The diffeomorphism $g \in \mathcal{G}$ can be precomposed by any $h \in \mathcal{G}$ satisfying $\left.h^{(\infty)}\right|_{z} \in \mathcal{G}_{\left.\mathrm{j}_{\infty} S\right|_{z}}^{(\infty)}$ and composed by $\bar{h} \in \mathcal{G}$ with $\left.\bar{h}^{(\infty)}\right|_{\bar{z}} \in \mathcal{G}_{\left.\mathrm{j}_{\infty} \bar{S}\right|_{\bar{z}}}^{(\infty)}$ to obtain a new equivalence map $\bar{g}=\bar{h} \circ g \circ h$.

\subsection{Equivalence of coframes}

In Cartan's framework, local equivalence problems are solved by recasting them as equivalence problems of coframes. We briefly explain how this is related to the equivalence problem of submanifolds. Let $\mathcal{H}$ be a Lie pseudo-group acting on a $p$-dimensional manifold $X$, and let

$$
\boldsymbol{\omega}=\left\{\omega^{i}=\sum_{j=1}^{p} u_{j}^{i}(x) d x^{j}, i=1, \ldots, p\right\}, \quad \overline{\boldsymbol{\omega}}=\left\{\bar{\omega}^{i}=\sum_{j=1}^{p} \bar{u}_{j}^{i}(\bar{x}) d \bar{x}^{j}, i=1, \ldots, p\right\}
$$


be two coframes on $X$ adapted to a given equivalence problem. We refer the reader to [10, 26] for a detailed account as to how to formulate equivalence problems using coframes. The local equivalence problem for the coframes (4.2) consists of determining whether there exists or not a local diffeomorphism $\varphi \in \mathcal{H}$ such that

$$
d \varphi^{*}\left(\bar{\omega}^{i}\right)=\sum_{j=1}^{p} h_{j}^{i}(x) \omega^{j}, \quad i=1, \ldots, p .
$$

The matrix $\left(h_{j}^{i}(x)\right) \in G L(p)$ in (4.3) is contained in a Lie group $H$ called the structure group of the equivalence problem. As in the equivariant moving frame approach, the primary goal of Cartan's equivalence method is to reduce the $H$-structure to an $\{e\}$-structure by normalizing the group coefficients $h_{j}^{i}$ after which it is possible to determine if the two coframes (4.2) are locally equivalent.

Since coframes on $X$ are local sections of the coframe bundle $\mathcal{F}(X)$, the equivalence problem of coframes can be interpreted as an equivalence problem of $p$-dimensional sections in $\mathcal{F}(X)$. Let $M \subset \mathcal{F}(X)$ be the subbundle of all coframes (4.2) adapted to a given equivalence problem. The action of $\mathcal{H}$ on $X$ naturally induces a Lie pseudo-group action $\mathcal{G} \subset \mathcal{H}^{(1)}$ on the subbundle $M$ via the equivalence criterion (4.3). Two coframes $\boldsymbol{\omega}, \overline{\boldsymbol{\omega}}$ are then locally equivalent if and only if their corresponding sections $S, \bar{S} \subset M$ are equivalent up to a local diffeomorphism $g \in \mathcal{G}$.

There are three possible outcomes to the coframe equivalence problem, each having their counterparts in the equivariant moving frame method. Borrowing the terminology used in [26], after a series of normalizations and prolongations, the three outcomes to Cartan's algorithm are:

a) Complete normalization: The $H$-structure reduces to an $\{e\}$-structure. This occurs when a section in $M \subset \mathcal{F}(X)$ is regular and lies in the domain of definition of a moving frame.

b) Prolongation: The coframe is prolonged to a larger space on which the equivalence problem reduces to an $\{e\}$-structure. This situation occurs when a section $S \subset M$ is singular and the isotropy group $\mathcal{G}_{\left.\mathrm{j}_{\infty} S\right|_{z}}^{(\infty)}$ is finite-dimensional.

c) Involution: The structure equations of the (possibly prolonged) coframe are in involution with nonzero (reduced) Cartan characters. This happens when a section $S \subset M$ is singular and the isotropy group $\mathcal{G}_{\left.\mathrm{j}_{\infty} S\right|_{z}}^{(\infty)}$ is infinite-dimensional.

We now illustrate each outcome with an example. At the same time we indicate some links between Cartan's approach and the equivariant moving frame method.

\subsubsection{Complete normalization}

Let $\mathcal{H}$ be a Lie pseudo-group acting on a manifold $X$ with local coordinates $x=\left(x^{1}, \ldots, x^{p}\right)$ and $\mathcal{G} \subset \mathcal{H}^{(1)}$ the induced pseudo-group action on the subbundle of coframes $M \subset \mathcal{F}(X)$ adapted to an equivalence problem. Given a moving frame $\widehat{\rho}_{i}: \mathcal{V}_{i}^{\infty} \rightarrow \mathcal{B}_{i}^{(\infty)}$ and a regular section $S \subset M$ with $\mathrm{j}_{\infty} S \subset \mathcal{V}_{i}^{\infty}$, an invariant coframe on $X$ is obtained by restricting the invariant horizontal 1-forms $\omega^{i}=\iota\left(d x^{i}\right)$ to $S$ :

$$
\boldsymbol{\omega}=\left.\boldsymbol{\omega}\right|_{S}=\left.\left\{\omega^{j} \equiv \widehat{\rho}_{i}^{*}\left(d_{J} X^{j}\right)=\iota\left(d x^{j}\right)\right\}\right|_{S} .
$$

The coframe derivatives are then given by the invariant derivative operators

$$
\mathcal{D}_{j}=\left.\mathcal{D}_{j}\right|_{S}=\frac{\partial}{\partial \omega^{j}}, \quad j=1, \ldots, p .
$$

Provided the cross-section defining the equivariant moving frame $\widehat{\rho}_{i}$ is compatible with the normalizations leading to an $\{e\}$-structure in Cartan's coframe method, the coframe (4.4) will be equivalent to the one obtained via Cartan's procedure. 
In Section 3, we saw that the structure equations for the coframe (4.4) can be deduced from the universal recurrence relation (3.14). In preparation for the next section, it is more convenient to recover these equations from the structure equations (2.10) of the equivalence pseudo-group. Let $z=(x, u)$ be local coordinates on $M \simeq X \times U$. Then, comparing the identities $d Z^{a}=\sigma^{a}+\mu^{a}$, $a=1, \ldots, m$, with the order 0 recurrence relations

$$
d X^{i} \equiv \omega^{i}+\mu^{i}, \quad i=1, \ldots, p, \quad d U^{\alpha} \equiv \sum_{j=1}^{p} U_{j}^{\alpha} \omega^{j}+\mu^{p+\alpha}, \quad \alpha=1, \ldots, q,
$$

we see that

$$
\sigma^{i} \equiv \omega^{i} \quad \text { while } \quad \sigma^{p+\alpha} \equiv \sum_{j=1}^{p} U_{j}^{\alpha} \omega^{j}
$$

Hence, pulling-back the structure equations for $\sigma^{1}, \ldots, \sigma^{p}$ in (2.10) by the moving frame $\widehat{\rho}_{i}$ we obtain the structure equations for the coframe (4.4).

Remark 4.12. The pull-back by $\widehat{\rho}_{i}$ of the remaining structure equations for $\sigma^{p+1}, \ldots, \sigma^{m}$ and the Maurer-Cartan forms $\mu_{B}^{a}$ yields syzygies among the normalized invariants. As these syzygies can be recovered from the recurrence relations (3.15), we can ignore these normalized structure equations.

Example 4.13. To make some analogies between the equivariant moving frame method and Cartan's coframe approach, the pseudo-group jets need to be recursively normalized in the equivariant formalism, [32]. We illustrate this by considering the generic branch I) of Example 3.13. Based on the cross-section (3.25) a moving frame is obtained by solving the normalization equations

$$
\begin{aligned}
& X=U=P=Q_{P^{4}}=Q_{P^{5}}=Q_{P^{4} U}=Q_{P^{4} X}=0, \quad Q_{P^{2} X^{2}}=1, \\
& Q_{U^{j} X^{k}}=Q_{P U^{j} X^{k}}=Q_{P^{2} U^{j}}=Q_{P^{2} U^{j} X}=Q_{P^{3} U^{j}}=Q_{P^{3} U^{j} X}=0, \quad j, k \geq 0 .
\end{aligned}
$$

In the recursive moving frame construction, the idea is to normalize the submanifold jets (or solve the normalization equations) in stages. For example, at order 0 we solve the normalization equations

$$
X=U=P=Q=0
$$

to obtain

$$
\chi=0, \quad \psi=0, \quad \psi_{x}=-p \psi_{u}, \quad \psi_{x x}=-\left(q \psi_{u}+p^{2} \psi_{u u}+2 p \psi_{x u}\right) .
$$

Then the (partially) normalized coframe (4.4) yields the $G$-structure

$$
\left(\begin{array}{c}
\omega^{x} \\
\omega^{u} \\
\omega^{p}
\end{array}\right)=\left(\begin{array}{ccc}
D_{x} \chi & \chi_{u} & 0 \\
0 & \psi_{u} & 0 \\
0 & \frac{D_{x} \psi_{u}}{D_{x} \chi} & \frac{\psi_{u}}{D_{x} \chi}
\end{array}\right)\left(\begin{array}{c}
d x \\
d u-p d x \\
d p-q d x
\end{array}\right)
$$

which is the starting point of Cartan's method. The next step in Cartan's algorithm is to compute the structure equations for the 1 -forms (4.7). For the problem at hand, the resulting structure equations are then prolonged, [26, p. 403]. To obtain the same structure equations, using the equivariant formalism, we must normalize

$$
Q_{U^{j} X^{k}}=Q_{P U^{j} X^{k}}=0, \quad j, k \geq 0 .
$$


The recurrence relations (3.13) are then used to obtain expressions for the (partially) normalized Maurer-Cartan forms $\mu, \nu, \nu_{X}, \mu_{U^{j} X^{k+2}}, \nu_{U^{j} X^{k+2}}, j, k \geq 0$. For example, the low order normalized Maurer-Cartan forms are

$$
\begin{aligned}
& \mu \equiv-\omega^{x}, \quad \nu \equiv-\omega^{u}, \quad \nu_{X} \equiv-\omega^{p} \\
& \nu_{X X} \equiv \nu_{U X X} \equiv \nu_{X X X} \equiv 0, \quad \mu_{X X} \equiv 2 \nu_{U X}+Q_{P P} \omega^{p} .
\end{aligned}
$$

Substituting these expressions into the structure equations (2.20), and recalling equality (4.5), we obtain

$$
\begin{aligned}
& d \omega^{x} \equiv \mu_{X} \wedge \omega^{x}+\mu_{U} \wedge \omega^{u}, \\
& d \omega^{u} \equiv \nu_{U} \wedge \omega^{u}+\omega^{x} \wedge \omega^{p}, \\
& d \omega^{p} \equiv \nu_{U X} \wedge \omega^{u}+\left(\nu_{U}-\mu_{X}\right) \wedge \omega^{p}, \\
& d \mu_{X} \equiv-2 \nu_{U X} \wedge \omega^{x}-\mu_{U X} \wedge \omega^{u}-\mu_{U} \wedge \omega^{p}+Q_{P P} \omega^{x} \wedge \omega^{p}, \\
& d \mu_{U} \equiv-\mu_{U U} \wedge \omega^{u}-\mu_{U X} \wedge \omega^{x}+\mu_{U} \wedge\left(\nu_{U}-\mu_{X}\right), \\
& d \nu_{U} \equiv-\nu_{U X} \wedge \omega^{x}+\mu_{U} \wedge \omega^{p}-\nu_{U U} \wedge \omega^{u}, \\
& d \nu_{U X} \equiv-\nu_{U U X} \wedge \mu^{u}+\left(\mu_{U X}-\nu_{U U}\right) \wedge \omega^{p}+\nu_{U X} \wedge \mu_{X} .
\end{aligned}
$$

These equations are equivalent to the ones obtained using Cartan's method, [26, pp. 403-404]. The correspondence is given by

$$
\begin{aligned}
& \theta^{1} \leftrightarrow \omega^{u}, \quad \theta^{2} \leftrightarrow \omega^{p}, \quad \theta^{3} \leftrightarrow \omega^{x}, \\
& \pi^{1} \leftrightarrow \nu_{U}, \quad \pi^{2} \leftrightarrow \nu_{U X}, \quad \pi^{4} \leftrightarrow \mu_{U}, \quad \pi^{6} \leftrightarrow \mu_{X}, \\
& \rho^{1} \leftrightarrow-\nu_{U U}, \quad \rho^{2} \leftrightarrow-\nu_{U U X}, \quad \rho^{3} \leftrightarrow-\mu_{U X}, \quad \rho^{4} \leftrightarrow-\mu_{U U}, \quad T \leftrightarrow-Q_{P P} .
\end{aligned}
$$

The next step in Cartan's algortithm is to normalize $Q_{P P}=0$. In the equivariant setting, we now set $Q_{P P U^{j}}=0, j \geq 0$, which leads to the normalization of the Maurer-Cartan forms $\mu_{U^{j+1} X}$. For example, the first normalized Maurer-Cartan form is

$$
\mu_{U X}=\frac{1}{4}\left(Q_{P P P} \omega^{p}+Q_{P P X} \omega^{x}+2 \nu_{U U}\right),
$$

which we then substitute in (4.8). The invariants $Q_{P P P}$ and $Q_{P P X}$ in (4.9) are essential torsion coefficients of the resulting structure equations and the next iteration of Cartan's algorithm is to normalize these invariants to zero. In the equivariant moving frame framework we now set $Q_{P^{3} U^{j}}=Q_{P^{2} U^{j} X}=Q_{P^{3} U^{j} X}=0, j \geq 0$, and normalize the Maurer-Cartan forms $\mu_{U^{j+2}}$, $\nu_{U^{j+2} X}, \nu_{U^{j+3}}, j \geq 0$. At this point we have recovered the universal normalizations (3.20) and all the Maurer-Cartan forms are normalized except for (3.21). From the recurrence relations, the low order normalized Maurer-Cartan forms are

$$
\begin{aligned}
& \mu \equiv-\omega^{x}, \quad \nu \equiv-\omega^{u}, \quad \nu_{X} \equiv-\omega^{p}, \quad \nu_{X X} \equiv 0 \\
& \mu_{X X} \equiv 2 \nu_{U X}, \quad \mu_{U X} \equiv \frac{1}{2} \nu_{U U}, \quad \mu_{U U} \equiv \frac{1}{6} Q_{P^{4} \omega^{p}}, \quad \nu_{U X X} \equiv 0, \\
& \nu_{U X X} \equiv \frac{1}{6} Q_{P^{2} X^{2} \omega^{x},}, \quad \nu_{U U U} \equiv \frac{1}{3}\left(Q_{P^{4} X} \omega^{p}+Q_{P^{3} X^{2}} \omega^{x}\right) .
\end{aligned}
$$

Substituting (4.10) into the structure equations (2.20) we obtain the structure equations for the eight-dimensional invariant coframe $\left\{\omega^{x}, \omega^{u}, \omega^{p}, \mu_{X}, \mu_{U}, \nu_{U}, \nu_{U X}, \nu_{U U}\right\}$ :

$$
\begin{aligned}
d \omega^{x} & \equiv \mu_{X} \wedge \omega^{x}+\mu_{U} \wedge \omega^{u} \\
d \omega^{u} & \equiv \nu_{U} \wedge \omega^{u}+\omega^{x} \wedge \omega^{p}
\end{aligned}
$$




$$
\begin{aligned}
& d \omega^{p} \equiv \nu_{U X} \wedge \omega^{u}+\left(\nu_{U}-\mu_{X}\right) \wedge \omega^{p}, \\
& d \mu_{X} \equiv-\frac{1}{2} \nu_{U U} \wedge \omega^{y}-2 \nu_{U X} \wedge \omega^{x}-\mu_{U} \wedge \omega^{p}, \\
& d \mu_{U} \equiv-\frac{1}{2} \nu_{U U} \wedge \omega^{x}+\frac{1}{6} Q_{P^{4}} \omega^{u} \wedge \omega^{p}+\left(\mu_{X}-\nu_{U}\right) \wedge \mu_{U}, \\
& d \nu_{U} \equiv-\nu_{U U} \wedge \omega^{u}-\nu_{U X} \wedge \omega^{x}+\mu_{U} \wedge \omega^{p}, \\
& d \nu_{U X} \equiv-\frac{1}{2} \nu_{U U} \wedge \omega^{p}+\nu_{U X} \wedge \mu_{X}+\frac{1}{6} Q_{P^{2} X^{2}} \omega^{u} \wedge \omega^{x}, \\
& d \nu_{U U} \equiv 2 \nu_{U X} \wedge \mu_{U}+\nu_{U U} \wedge \nu_{U}+\frac{1}{3} Q_{P^{4} X} \omega^{u} \wedge \omega^{p}+\frac{1}{3} Q_{P^{3} X^{2} \omega^{u} \wedge \omega^{x} .}
\end{aligned}
$$

These equations are equivalent to Cartan's structure equations [26, equation (12.72)]. The correspondence is given by

$$
\begin{aligned}
& \omega^{x} \leftrightarrow \theta^{3}, \quad \omega^{u} \leftrightarrow \theta^{1}, \quad \omega^{p} \leftrightarrow \theta^{2}, \\
& \mu_{X} \leftrightarrow \pi^{6}, \quad \mu_{U} \leftrightarrow \pi^{4}, \quad \nu_{U} \leftrightarrow \pi^{1}, \quad \nu_{U X} \leftrightarrow \pi^{2}, \quad \nu_{U U} \leftrightarrow-\rho, \\
& Q_{P^{4}} \leftrightarrow 6 K_{1}, \quad Q_{P^{2} X^{2}} \leftrightarrow 6 K_{2}, \quad Q_{P^{4} X} \leftrightarrow 3 K_{3}, \quad Q_{P^{3} X^{2}} \leftrightarrow 3 K_{4} .
\end{aligned}
$$

The structure equations (4.11) and identity $d \circ d=0$ imply that $Q_{P^{3} X^{2}}=\mathcal{D}_{P} Q_{P^{2} X^{2}}$ and $Q_{P^{4} X}=$ $\mathcal{D}_{X} Q_{P^{4}}$. We note that these differential relations can also be deduced from the recurrence relations (3.24).

At this stage, the equivalence problem splits into the branches identified in Example 3.13. For the generic branch, corresponding to Case I), we can normalize the remaining pseudogroup parameters by making the normalizations (4.6). To obtain the structure equations of the invariant coframe $\left\{\omega^{x}, \omega^{u}, \omega^{p}\right\}$ we use the recurrence relations to find the expressions for the low order normalized Maurer-Cartan forms

$$
\begin{aligned}
& \mu \equiv-\omega^{x}, \quad \nu \equiv-\omega^{u}, \quad \nu_{X} \equiv-\omega^{p}, \quad \mu_{U} \equiv-\frac{1}{5}\left(Q_{P^{5} X} \omega^{x}+Q_{P^{5} U} \omega^{u}+Q_{P^{6} \omega^{p}}\right),
\end{aligned}
$$

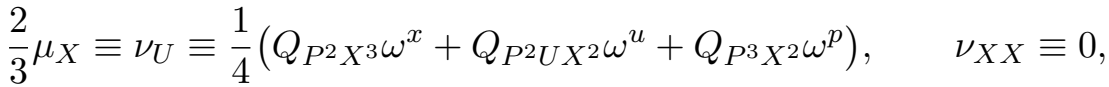

$$
\begin{aligned}
& \nu_{U X} \equiv-\left(Q_{P^{4} X^{2}} \omega^{x}+Q_{P^{4} U X} \omega^{u}+Q_{P^{5} X} \omega^{p}\right) \text {. }
\end{aligned}
$$

Substituting (4.12) in the first three structure equations of (4.11) we obtain

$$
\begin{aligned}
& d \omega^{x} \equiv\left(\frac{3}{8} Q_{P^{2} U X^{2}}+\frac{1}{5} Q_{P^{5} X}\right) \omega^{u} \wedge \omega^{x}+\frac{3}{8} Q_{P^{3} X^{2}} \omega^{p} \wedge \omega^{x}+\frac{1}{5} Q_{P^{6}} \omega^{u} \wedge \omega^{p}, \\
& d \omega^{u} \equiv \omega^{x} \wedge \omega^{p}+\frac{1}{4} Q_{P^{2} X^{3} \omega^{x}} \wedge \omega^{u}+\frac{1}{4} Q_{P^{3} X^{2}} \omega^{p} \wedge \omega^{u}, \\
& d \omega^{p} \equiv\left(Q_{P^{5} X}-\frac{1}{8} Q_{P^{2} U X^{2}}\right) \omega^{u} \wedge \omega^{p}+Q_{P^{4} X^{2}} \omega^{u} \wedge \omega^{x}+\frac{1}{8} Q_{P^{2} X^{3} \omega^{p}} \wedge \omega^{x} .
\end{aligned}
$$

Extracting the invariants from the structure coefficients, it follows from Cartan's moving frame theory that the normalized invariants

$$
Q_{P^{3} X^{2}}, \quad Q_{P^{2} U X^{2}}, \quad Q_{P^{2} X^{3}}, \quad Q_{P^{6}}, \quad Q_{P^{5} X}, \quad Q_{P^{4} X^{2}}
$$

form a generating set for the algebra of differential invariants. We note that the generating set (4.13) does contain all the normalized invariants of order $\leq 6$. Based on the normalizations (4.6), the complete list of normalized invariants of order $\leq 6$ is

$$
\begin{array}{lcccc}
Q_{P^{3} X^{2}}, & Q_{P^{2} U X^{2}}, & Q_{P^{2} X^{3}}, & Q_{P^{6}}, & \\
Q_{P^{5} U}, & Q_{P^{5} X}, & Q_{P^{4} U^{2}}, & Q_{P^{4} U X}, & Q_{P^{4} X^{2}} .
\end{array}
$$


Using, as always, the recurrence relations we can check that the extra invariants $Q_{P^{5} U}, Q_{P^{4} U^{2}}$, $Q_{P^{4} U X}$ can be expressed in terms of (4.13) and their invariant derivatives:

$$
\begin{aligned}
& Q_{P^{5} U}=\frac{5}{6}\left[\mathcal{D}_{U} Q_{P^{3} X^{2}}-\mathcal{D}_{P} Q_{P^{2} U X^{2}}-\frac{3}{10} Q_{P^{3} X^{2}} Q_{P^{2} U X^{2}}+Q_{P^{5} X} Q_{P^{3} X^{2}}+\frac{1}{5} Q_{P^{6}} Q_{P^{2} X^{3}}\right] \\
& Q_{P^{4} U X}=\frac{1}{6}\left[\mathcal{D}_{U} Q_{P^{2} X^{3}}-\mathcal{D}_{X} Q_{P^{2} U X^{2}}+\frac{1}{8} Q_{P^{2} U X^{2}} Q_{P^{2} X^{3}}+Q_{P^{4} X^{2}} Q_{P^{3} X^{2}}+\frac{1}{5} Q_{P^{5} X} Q_{P^{2} X^{3}}\right], \\
& Q_{P^{4} U^{2}}=\frac{4}{5}\left[\mathcal{D}_{U} Q_{P^{5} X}-\mathcal{D}_{X} Q_{P^{5} U}+\frac{1}{4} Q_{P^{5} X} Q_{P^{2} U X^{2}}+\frac{1}{5} Q_{P^{5} X}^{2}+Q_{P^{6}} Q_{P^{4} X^{2}}-\frac{1}{8} Q_{P^{5} U} Q_{P^{2} X^{3}}\right] .
\end{aligned}
$$

The structure equations for the invariant coframe $\left\{\omega^{x}, \omega^{u}, \omega^{p}\right\}$ and the determination of a generating set of invariants for the other regular cases of Example 3.13 are obtained in a similar fashion.

\subsubsection{Prolongation}

We now assume that a section $S \subset M \subset \mathcal{F}(X)$ is singular with $\mathrm{j}_{\infty} S \subset \mathcal{S}_{i}^{\infty}$. Also, we consider the case when the isotropy group $\mathcal{G}_{\left.\mathrm{j}_{\infty} S\right|_{z}}^{(\infty)}$ is finite-dimensional and locally parametrized by $g=$ $\left(g_{1}, \ldots, g_{r}\right)$.

Let $\boldsymbol{\mu}=\left\{\mu^{1}, \ldots, \mu^{r}\right\}$ be the Maurer-Cartan forms associated with the isotropy group parameters $g=\left(g_{1}, \ldots, g_{r}\right)$. Then the invariant horizontal 1-forms (4.4) together with the MaurerCartan forms $\boldsymbol{\mu}=\left.\widehat{\rho}_{i}^{*}(\boldsymbol{\mu})\right|_{S}$ constitute an invariant coframe on the prolonged bundle $\left.\mathcal{P}_{i}^{(\infty)}\right|_{S}$. As in the previous section, the structure equations of the prolonged coframe $\{\boldsymbol{\omega}, \boldsymbol{\mu}\}$ are obtained by pulling-back the structure equations of the equivalence pseudo-group by the partial moving frame $\widehat{\rho}_{i}$.

Example 4.14. In Example 3.18 we saw that if the fourth order (relative) invariants $Q_{P^{4}} \equiv$ $Q_{P^{2} X^{2}} \equiv 0$ are identically zero then all higher order invariants (3.22) are also equal to zero. Hence, in Case IV) the only invariants are constant functions, and two singular second order ordinary differential equations are equivalent under point transformations, [5, 10, 26, 40].

Setting $Q_{P^{4}} \equiv Q_{P^{2} X^{2}} \equiv Q_{P^{4} X} \equiv Q_{P^{3} X^{2}} \equiv 0$ in the structure equations (4.11) we obtain the Maurer-Cartan structure equations for the eighth-dimensional symmetry group of point transformations (isomorphic to SL(3)) of a singular second order ordinary differential equation:

$$
\begin{aligned}
d \omega^{x} & \equiv \mu_{X} \wedge \omega^{x}+\mu_{U} \wedge \omega^{u}, \\
d \omega^{u} & \equiv \nu_{U} \wedge \omega^{u}+\omega^{x} \wedge \omega^{p}, \\
d \omega^{p} & \equiv \nu_{U X} \wedge \omega^{u}+\left(\nu_{U}-\mu_{X}\right) \wedge \omega^{p}, \\
d \mu_{U} & \equiv-\frac{1}{2} \nu_{U U} \wedge \omega^{x}+\left(\mu_{X}-\nu_{U}\right) \wedge \mu_{U}, \\
d \nu_{U} & \equiv \mu_{U} \wedge \omega^{p}-\nu_{U X} \wedge \omega^{x}-\nu_{U U} \wedge \omega^{u}, \\
d \mu_{X} & \equiv-2 \nu_{U X} \wedge \omega^{x}-\frac{1}{2} \nu_{U U} \wedge \omega^{u}-\mu_{U} \wedge \omega^{p}, \\
d \nu_{U U} & \equiv 2 \nu_{U X} \wedge \mu_{U}+\nu_{U U} \wedge \nu_{U}, \\
d \nu_{U X} & \equiv \nu_{U X} \wedge \mu_{X}-\frac{1}{2} \nu_{U U} \wedge \omega^{p} .
\end{aligned}
$$

Restricting (4.14) to a fixed point $(x, u, p)$ we obtain the structure equations

$$
\begin{aligned}
& d \mu_{X} \equiv 0, \quad d \mu_{U} \equiv\left(\mu_{X}-\nu_{U}\right) \wedge \mu_{U}, \quad d \nu_{U} \equiv 0 \\
& d \nu_{U U} \equiv 2 \nu_{U X} \wedge \mu_{U}+\nu_{U U} \wedge \nu_{U}, \quad d \nu_{U X} \equiv \nu_{U X} \wedge \mu_{X},
\end{aligned}
$$


for the five-dimensional isotropy group $\mathcal{G}_{z}^{(\infty)}($. Identifying the jets of a local vector field (2.7) with the coefficients of its Taylor expansion, [33, 42]:

$$
\left.\mathrm{j}_{\infty} \mathbf{v}\right|_{z_{0}} \simeq \sum_{a=1}^{b} \sum_{\# B \geq 0} \zeta_{B}^{a}\left(z_{0}\right) \frac{\left(z-z_{0}\right)^{B}}{B !} \frac{\partial}{\partial z^{a}}
$$

the fiber $\left.\mathrm{J}^{\infty} T M\right|_{z_{0}}$ inherits a Lie algebra structure. The monomial vector fields

$$
\mathbf{v}_{a}^{B}=\frac{\left(z-z_{0}\right)^{B}}{B !} \frac{\partial}{\partial z^{a}}, \quad \# B \geq 0, \quad a=1, \ldots, m,
$$

provide a basis of the vector space $\left.\mathrm{J}^{\infty} T M\right|_{z_{0}}$ and they can be identified as dual vectors to the Maurer-Cartan forms $\left.\mu_{B}^{a}\right|_{z_{0}}$. Choosing, for simplicity, $z_{0}=\left(x_{0}, u_{0}, p_{0}, q_{0}\right)=0$, the isotropy Lie algebra at the origin is spanned by the infinitesimal generators ${ }^{2}$

$$
\begin{aligned}
& \mathbf{v}_{x}^{x}=x \frac{\partial}{\partial x}, \quad \mathbf{v}_{u}^{x}=u \frac{\partial}{\partial x}, \quad \mathbf{v}_{u}^{u}=u \frac{\partial}{\partial u}, \\
& \mathbf{v}_{u x}^{u}=x^{2} \frac{\partial}{\partial x}+x u \frac{\partial}{\partial u}, \quad \mathbf{v}_{u u}^{u}=\frac{x u}{2} \frac{\partial}{\partial x}+\frac{u^{2}}{2} \frac{\partial}{\partial u},
\end{aligned}
$$

and satisfy commutation relations dual to (4.15). That is,

$$
\begin{array}{lll}
{\left[\mathbf{v}_{x}^{x}, \mathbf{v}_{u x}^{u}\right]=\mathbf{v}_{u x}^{u},} & {\left[\mathbf{v}_{u}^{x}, \mathbf{v}_{u x}^{u}\right]=2 \mathbf{v}_{u u}^{u},} & {\left[\mathbf{v}_{u}^{u}, \mathbf{v}_{u u}^{u}\right]=\mathbf{v}_{u u}^{u},} \\
{\left[\mathbf{v}_{u}^{x}, \mathbf{v}_{x}^{x}\right]=\mathbf{v}_{u}^{x},} & {\left[\mathbf{v}_{u}^{u}, \mathbf{v}_{u}^{x}\right]=\mathbf{v}_{u}^{x} .} &
\end{array}
$$

The corresponding 5 -dimensional isotropy group is generated by the local transformations

$$
\begin{aligned}
& (X, U)=\left(\lambda_{1} x, u\right), \quad(X, U)=\left(x+\epsilon_{1} u, u\right), \quad(X, U)=\left(x, \lambda_{2} u\right), \\
& (X, U)=\left(\frac{x}{1-\epsilon_{2} x}, \frac{u}{1-\epsilon_{2} x}\right), \quad(X, U)=\left(\frac{x}{1-\epsilon_{3} u}, \frac{u}{1-\epsilon_{3} u}\right),
\end{aligned}
$$

which can be prolonged to $p=u_{x}, q=u_{x x}$.

\subsubsection{Involution}

We now consider the case when the isotropy group is infinite-dimensional. As in the preceding section, let $g^{(n)}=\left(g_{1}, \ldots, g_{r_{n}}\right)$ denote the pseudo-group jets parametrizing $\mathcal{G}_{\left.\mathrm{j}_{n} S\right|_{z}}^{(n)}$ and let $\boldsymbol{\mu}^{(n)}$ denote the corresponding Maurer-Cartan forms. Since the isotropy group is infinite-dimensional, the collection of 1-forms $\left\{\boldsymbol{\omega}, \boldsymbol{\mu}^{(n)}\right\}$ does not form a coframe on the prolonged bundle $\left.\mathcal{P}_{i}^{(n)}\right|_{S}$. Nevertheless, the structure equations for the 1-forms $\left\{\boldsymbol{\omega}, \boldsymbol{\mu}^{(n)}\right\}$ are still obtained by pulling-back the structure equations of the equivalence pseudo-group by the partial moving frame.

Example 4.15. In this example we consider the local equivalence of second order ordinary differential equations (2.11) under the pseudo-group of contact transformations (2.12). Taking into account the linear relations (2.16) among the Maurer-Cartan forms, the recurrence relations (3.13) for the lifted invariants are

$$
\begin{aligned}
d X \equiv & \omega^{x}+\mu^{x}, \quad d U \equiv \omega^{u}+\mu^{u}, \quad d X^{p} \equiv \omega^{p}+\mu^{p} \\
d Q_{J} \equiv & Q_{J, X} \omega^{x}+Q_{J, U} \omega^{u}+Q_{J, P} \omega^{p}+\mu_{J, X}^{p} \\
& +\mathbb{D}_{J}\left[P \mu_{U}^{p}+Q \mu_{P}^{p}-Q\left(\mu_{X}^{x}+P \mu_{U}^{x}+Q \mu_{P}^{x}\right)\right. \\
& \left.-\mu^{x} Q_{X}-\mu^{u} Q_{U}-\mu^{p} Q_{P}\right]+\mu^{x} Q_{J, X}+\mu^{u} Q_{J, U}+\mu^{P} Q_{J, P} .
\end{aligned}
$$

\footnotetext{
${ }^{2}$ The infinitesimal generators are prolonged to $p=u_{x}$ and $q=u_{x x}$ using formula (3.10).
} 
Since the Maurer-Cartan forms $\mu_{J, X}^{p}$ are linearly independent, it follows from (4.16) that the equivalence pseudo-group is transitive on $\mathrm{J}^{\infty}$. Also, the inequality $\operatorname{dim} \mathcal{G}^{(n)}>\operatorname{dim} \mathrm{J}^{n}$ for all $n$ implies that the prolonged action is nowhere free and all submanifold jets are singular so that $\mathcal{S}^{\infty}=\mathrm{J}^{\infty}$. Choosing the cross-section

$$
\mathcal{K}^{\infty}=\left\{x=u=p=q_{J}=0, \# J \geq 0\right\},
$$

the normalized Maurer-Cartan forms are

$$
\mu^{x} \equiv-\omega^{x}, \quad \mu^{u} \equiv-\omega^{u}, \quad \mu^{p} \equiv-\omega^{p}, \quad \mu_{J, X}^{p} \equiv 0 .
$$

The structure equations for the invariant 1-forms $\boldsymbol{\omega}=\left\{\omega^{x}, \omega^{u}, \omega^{p}\right\}$ are obtained by substituting $\sigma^{i} \equiv-\mu^{i} \equiv \omega^{i}, 1 \leq i \leq 3$, into the structure equations (2.17). Taking into account that (2.16) implies $\mu_{U}^{p}=\mu_{U X}^{u}$ when $P=0$, we obtain

$$
\begin{aligned}
d \omega^{x} & \equiv \mu_{X}^{x} \wedge \omega^{x}+\mu_{U}^{x} \wedge \omega^{u}+\mu_{P}^{x} \wedge \omega^{p}, \\
d \omega^{u} & \equiv \mu_{U}^{u} \wedge \omega^{u}+\omega^{x} \wedge \omega^{p}, \\
d \omega^{p} & \equiv \mu_{X U}^{u} \wedge \omega^{u}+\left(\mu_{U}^{u}-\mu_{X}^{x}\right) \wedge \omega^{p} .
\end{aligned}
$$

These equations are equivalent to the structure equations [26, equation (11.5)] obtained using Cartan's approach. The correspondence is given by

$$
\begin{aligned}
& \theta^{1} \leftrightarrow \omega^{u}, \quad \theta^{2} \leftrightarrow \omega^{p}, \quad \theta^{3} \leftrightarrow \omega^{x}, \quad \pi^{1} \leftrightarrow \mu_{U}^{u}, \quad \pi^{2} \leftrightarrow \mu_{X U}^{u}, \\
& \pi^{3} \leftrightarrow \mu_{U}^{u}-\mu_{X}^{x}, \quad \pi^{4} \leftrightarrow \mu_{U}^{x}, \quad \pi^{5} \leftrightarrow \mu_{P}^{x} .
\end{aligned}
$$

By Cartan's involutivity test, [2, 15, 26], the structure equations (4.17) are involutive. In Section 6, following Seiler's book [35], Cartan's test based on the algebraic theory of involution is introduced. This offers an alternative way of verifying, for example, that the structure equations (4.17) are involutive.

\section{Algebra of differential invariants}

As seen in Section 4, the fundamental basis theorem is at the heart of the local equivalence problem solution. Following [28], we now introduce the algebraic constructions used to prove the fundamental basis theorem for Lie pseudo-groups acting freely and regularly on dense open subsets of $\mathrm{J}^{\infty}$. Once this is done we explain how to modify the algebraic constructions to take into account Lie pseudo-groups acting freely and regularly on invariant regular subbundles of $\mathrm{J}^{\infty}$. Further modifications will allow us to deal with Lie pseudo-groups acting regularly and nonfreely on invariant regular subbundles of $\mathrm{J}^{\infty}$. The main conclusion is that, with the appropriate modifications and regularity assumptions, the algebraic proof of the fundamental basis theorem given in [28] extends to Lie pseudo-groups acting regularly and freely (or non-freely) on invariant regular subbundles of $\mathrm{J}^{\infty}$.

\subsection{Regular submanifold jets}

Let $\mathcal{G}$ be a Lie pseudo-group acting on an $m$-dimensional manifold $M$. For the moment we restrict our considerations to a dense open subset $\mathcal{V}^{\infty} \subset \mathrm{J}^{\infty}$ where the action becomes free at order $n^{\star}$.

Let $\mathbb{R}[t, T]$ denote the algebra of real polynomials in the variables $t=\left(t_{1}, \ldots, t_{m}\right)$ and $T=$ $\left(T^{1}, \ldots, T^{m}\right)$, and define

$$
\mathcal{T}=\left\{\eta(t, T)=\sum_{a=1}^{m} \eta_{a}(t) T^{a}\right\} \simeq \mathbb{R}[t] \otimes \mathbb{R}^{m} \subset \mathbb{R}[t, T]
$$


to be the $\mathbb{R}[t]$ module consisting of homogeneous linear polynomials in the variable $T$. Let $\mathcal{T}^{n} \subset \mathcal{T}$ denote the subspace of homogeneous polynomials of degree $n$ in $t$. The notations $\mathcal{T} \leq n=\oplus_{k=0}^{n} \mathcal{T}^{k}$ and $\mathcal{T} \geq n=\oplus_{k=n}^{\infty} \mathcal{T}^{k}$ are used to denote the space of polynomials of degree $\leq n$ and $\geq n$ in $t$. Let $\mathbf{H}: \mathcal{T} \rightarrow \mathcal{T}$ be the highest order term operator defined by the requirement that for $0 \neq \eta \in \mathcal{T} \leq n$ with $0 \neq \mathbf{H}(\eta) \in \mathcal{T}^{n}$ the equality $\eta=\mathbf{H}(\eta)+\lambda$ holds for some $\lambda \in \mathcal{T} \leq n-1$. Locally, let $\left(\mathrm{J}^{\infty} T M\right)^{*} \simeq M \times \mathcal{T}$ via the pairing $\left\langle\mathrm{j}_{\infty} \mathbf{v} ; t_{B} T^{a}\right\rangle=\zeta_{B}^{a}$. Then, an $n^{\text {th }}$ order linear differential equation

$$
L\left(z, \zeta^{(n)}\right)=\sum_{a=1}^{m} \sum_{\# B \leq n} h_{a}^{B}(z) \zeta_{B}^{a}=0
$$

can be identified with the parametrized polynomial

$$
\eta(z ; t, T)=\sum_{a=1}^{m} \sum_{\# B \leq n} h_{a}^{B}(z) t_{B} T^{a} \in \mathcal{T}^{\leq n} .
$$

Definition 5.1. The symbol $\boldsymbol{\Sigma}(L)$ of the $n^{\text {th }}$ order (non-zero) linear differential equation (5.1) consists of the highest order terms in its defining polynomial (5.2):

$$
\Sigma\left[L\left(z, \zeta^{(n)}\right)\right]=\mathbf{H}[\eta(z ; t, T)]=\sum_{a=1}^{m} \sum_{\# B=n} h_{a}^{B}(z) t_{B} T^{a} \in \mathcal{T}^{n} .
$$

Let $\mathcal{L}=\left(\mathrm{J}^{\infty} \mathfrak{g}\right)^{\perp} \subset\left(\mathrm{J}^{\infty} T M\right)^{*}$ denote the annihilator subbundle of the infinitesimal generator jet bundle, and define

$$
\mathcal{I}=\mathbf{H}(\mathcal{L})
$$

to be the span of the highest order terms of the annihilating polynomials at each $z \in M$. Since the infinitesimal determining system (2.8) is formally integrable, it follows that the system is closed under differentiation with respect to the total derivative operators $\mathbb{D}_{z^{a}}$. At the symbol level, since total differentiation with respect to $\mathbb{D}_{z^{a}}$ corresponds to multiplication by $t_{a}$

$$
\boldsymbol{\Sigma}\left(\mathbb{D}_{z^{a}} L\right)=t_{a} \boldsymbol{\Sigma}(L), \quad a=1, \ldots, m,
$$

where $L$ is of the form (5.1), it follows that at each point $z \in M$ the fiber $\left.\mathcal{I}\right|_{z}$ forms a graded submodule of $\mathcal{T}$. This submodule is called the symbol module of the Lie pseudo-group at the point $z$.

We now introduce a second module called the prolonged symbol submodule of the prolonged infinitesimal generator (3.10). Introducing the variables $s=\left(s_{1} \ldots, s_{p}\right)$ and $S=\left(S^{1}, \ldots, S^{q}\right)$, let

$$
\widehat{\mathcal{S}}=\left\{\widehat{\sigma}(s, S)=\sum_{\alpha=1}^{q} \widehat{\sigma}_{\alpha}(s) S^{\alpha}\right\} \simeq \mathbb{R}[s] \otimes \mathbb{R}^{q}
$$

denote the $\mathbb{R}[s]$ module of polynomials that are linear in $S$. The module $\widehat{\mathcal{S}}$ is extended to

$$
\mathcal{S}=\mathbb{R}^{p} \oplus \widehat{\mathcal{S}}=\sum_{n=-1}^{\infty} \mathcal{S}^{n}
$$

by introducing $\mathcal{S}^{-1}=\left\{c \cdot \widetilde{s}=c_{1} \widetilde{s}_{1}+\cdots+c_{p} \widetilde{s}_{p}\right\} \simeq \mathbb{R}^{p}$, where $\widetilde{s}=\left(\widetilde{s}_{1}, \ldots, \widetilde{s}_{p}\right) \in \mathbb{R}^{p}$ are extra variables. The space $\mathcal{S}$ is endowed with the structure of an $\mathbb{R}[s]$ module by taking the usual module structure on $\widehat{\mathcal{S}}$ and setting

$$
\tau(s) \widetilde{s}_{i}=\tau(0) \widetilde{s}_{i} \quad \text { for any polynomial } \quad \tau(s) \in \mathbb{R}[s] .
$$


The space $\mathcal{S}$ is called the submanifold jet module. A highest order term operator $\mathbf{H}: \mathcal{S} \rightarrow \mathcal{S}$ is also introduced on $\mathcal{S}$. For $\sigma(\widetilde{s}, s, S)=c \cdot \widetilde{s}+\widehat{\sigma}(s, S)$ with $\widehat{\sigma}(s, S) \neq 0$ we require that

$$
\mathbf{H}[\sigma(\widetilde{s}, s, S)]=\mathbf{H}[\widehat{\sigma}(s, S)] .
$$

In other words, the elements of $\mathcal{S}^{-1}$ have zero highest order term. We also fix a convenient degree compatible term ordering on the monomials of $\mathcal{S}$. For example, one could choose the degree lexicographic ordering, [35].

Given an arbitrary vector field

$$
\mathbf{V}=\sum_{i=1}^{p} \xi^{i} \frac{\partial}{\partial x^{i}}+\sum_{\alpha=1}^{q} \sum_{\# J \geq 0} \phi_{\alpha}^{J} \frac{\partial}{\partial u_{J}^{\alpha}} \in T \mathrm{~J}^{\infty},
$$

whose coefficients do not have to be the coefficients of the prolonged vector field (3.10), the cotangent bundle $T^{*} \mathrm{~J}^{\infty}$ is identified with $\mathrm{J}^{\infty} \times \mathcal{S}$ via the pairing

$$
\left\langle\mathbf{V} ; \widetilde{s}_{i}\right\rangle=\xi^{i}, \quad\left\langle\mathbf{V} ; S^{\alpha}\right\rangle=Q^{\alpha}=\phi_{\alpha}-\sum_{i=1}^{p} u_{i}^{\alpha} \xi^{i}, \quad\left\langle\mathbf{V} ; s_{J} S^{\alpha}\right\rangle=\phi_{\alpha}^{J}, \quad \# J \geq 1 .
$$

Fixing $z^{(\infty)} \in \mathcal{V}^{\infty} \subset \mathrm{J}^{\infty}$ with $\pi_{0}^{\infty}\left(z^{(\infty)}\right)=z$, the prolongation map $\mathbf{p}=\mathbf{p}_{z(\infty)}^{(\infty)}:\left.\mathrm{J}^{\infty} T M\right|_{z} \rightarrow$ $\left.T \mathrm{~J}^{\infty}\right|_{z^{(\infty)}}$ given in (3.11) induces the dual prolongation map $\mathbf{p}^{*}: \mathcal{S} \rightarrow \mathcal{T}$ defined by

$$
\left\langle\mathrm{j}_{\infty} \mathbf{v} ; \mathbf{p}^{*}(\sigma)\right\rangle=\left\langle\mathbf{p}\left(\mathrm{j}_{\infty} \mathbf{v}\right) ; \sigma\right\rangle=\left\langle\mathbf{v}^{(\infty)} ; \sigma\right\rangle \quad \text { for all }\left.\mathrm{j}_{\infty} \mathbf{v} \in \mathrm{J}^{\infty} T M\right|_{z} \quad \text { and } \quad \sigma \in \mathcal{S} .
$$

Next, let

$$
\begin{aligned}
& \beta_{i}(t)=t_{i}+\sum_{\alpha=1}^{q} u_{i}^{\alpha} t_{p+\alpha}, \quad i=1, \ldots, p, \\
& B^{\alpha}(T)=T^{p+\alpha}-\sum_{i=1}^{p} u_{i}^{\alpha} T^{i}, \quad \alpha=1, \ldots, q,
\end{aligned}
$$

where $u_{i}^{\alpha}=\partial u^{\alpha} / \partial x^{i}$ are the first order jet coordinates of the fixed submanifold jet $z^{(\infty)}$. Geometrically, the polynomial $B^{\alpha}(T)$ is the symbol of the characteristic component $Q^{\alpha}$ appearing in (5.4) while $\beta_{i}(t)$ represents the symbol of the $i^{\text {th }}$ total derivative operator $D_{x^{i}}$ :

$$
\boldsymbol{\Sigma}\left(D_{x^{i}} L\right)=\beta_{i}(t) \boldsymbol{\Sigma}(L),
$$

where $L$ is any linear differential equation in the vector field jet coordinates. For fixed first order jet coordinates $u_{i}^{\alpha}$, the polynomials (5.5) define a linear map

$$
\beta: \mathbb{R}^{2 m} \rightarrow \mathbb{R}^{m} \quad \text { given by } \quad s_{i}=\beta_{i}(t), \quad S^{\alpha}=B^{\alpha}(T),
$$

and for $\widehat{\sigma}(s, S) \in \widehat{\mathcal{S}} \subset \mathcal{S}$ the equality

$$
\mathbf{H}\left[\mathbf{p}^{*}(\widehat{\sigma})\right]=\boldsymbol{\beta}^{*}[\mathbf{H}(\widehat{\sigma})]
$$

holds.

Definition 5.2. The prolonged annihilator subbundle of the prolonged Lie algebra $\mathfrak{g}^{(\infty)}$ at $z^{(\infty)} \in \mathcal{V}^{\infty}$ is

$$
\mathcal{Z}=\left(\left.\mathfrak{g}^{(\infty)}\right|_{z^{(\infty)}}\right)^{\perp}=\left.\left(\mathbf{p}^{*}\right)^{-1} \mathcal{L}\right|_{z} \subset \mathcal{S} .
$$

Furthermore, let

$$
\mathcal{U}=\mathbf{H}(\mathcal{Z}) \subset \mathcal{S}
$$

be the span of the highest order terms of the prolonged annihilator subbundle (in general, $\mathcal{U}$ is not a submodule). 
Definition 5.3. The prolonged symbol submodule is defined as the inverse image of the symbol module (5.3) under the polynomial pull-back morphism (5.7):

$$
\mathcal{J}=\left(\beta^{*}\right)^{-1}\left(\left.\mathcal{I}\right|_{z}\right)=\left\{\widehat{\sigma}(s, S) \in \widehat{\mathcal{S}}: \beta^{*}(\widehat{\sigma}(s, S))=\left.\widehat{\sigma}(\beta(t), B(T)) \in \mathcal{I}\right|_{z}\right\} \subset \widehat{\mathcal{S}}
$$

From (5.8) and (5.9), the containment $\mathcal{U} \subset \mathcal{J}$ always holds. When the action is locally free the containment becomes an equality, [28].

Lemma 5.4. Let $n^{\star}$ be the order of freeness. Then for all $n>n^{\star}$

$$
\left.\mathcal{U}^{n}\right|_{z^{(n)}}=\left.\mathcal{J}^{n}\right|_{z^{(n)}}, \quad z^{(n)} \in \mathcal{V}^{n} .
$$

Given a moving frame $\widehat{\rho}: \mathcal{V}^{\infty} \rightarrow \mathcal{B}^{(\infty)}$, the invariantization map is used to invariantize the preceding algebraic constructions. For example, the invariantization of a section

$$
\eta(x, u ; t, T)=\sum_{a=1}^{m} \sum_{\# B \leq n} h_{b}^{B}(x, u) t_{B} T^{a}
$$

of the annihilator bundle $\mathcal{L}$ is the polynomial

$$
\bar{\eta}(X, U ; t, T)=\iota[\eta(x, u ; t, T)]=\sum_{a=1}^{m} \sum_{\# B \leq n} h_{a}^{B}(X, U) t_{B} T^{a}
$$

obtained by replacing the coordinates $(x, u)$ on $M$ by their invariantizations $(X, U)=\iota(x, u)$. Similarly, the invariantization of a prolonged symbol polynomial

$$
\widehat{\sigma}\left(x, u^{(k)} ; s, S\right)=\sum_{\alpha=1}^{q} \sum_{\# J \leq n} h_{\alpha}^{J}\left(x, u^{(k)}\right) s_{J} S^{\alpha} \in \widehat{\mathcal{S}}^{\leq n}
$$

is the polynomial

$$
\bar{\sigma}\left(X, U^{(k)}, s, S\right)=\iota\left[\widehat{\sigma}\left(x, u^{(k)} ; s, S\right)\right]=\sum_{\alpha=1}^{q} \sum_{\# J \leq n} h_{\alpha}^{J}\left(X, U^{(k)}\right) s_{J} S^{\alpha} .
$$

Let $\left.\overline{\mathcal{U}}^{n}\right|_{z^{(n)}}=\left.\iota\left(\mathcal{U}^{n}\right)\right|_{z^{(n)}}$ and $\left.\overline{\mathcal{J}}^{n}\right|_{z^{(n)}}=\left.\iota\left(\mathcal{J}^{n}\right)\right|_{z^{(n)}}$. The equality $\left.\overline{\mathcal{U}}^{n}\right|_{z^{(n)}}=\left.\overline{\mathcal{J}}^{n}\right|_{z^{(n)}}$ for all $n>n^{\star}$ is the key to proving the fundamental basis theorem. Since $\left.\overline{\mathcal{J}}^{>n^{\star}}\right|_{z^{(n)}}$ is a polynomial ideal it has a Gröbner basis, [8], which brings algebraic structure into the problem. After identifying the polynomial (5.11) with the differential invariant

$$
I_{\bar{\sigma}}=\sum_{\alpha=1}^{q} \sum_{\# J \geq 0} h_{\alpha}^{J}\left(X, U^{(k)}\right) U_{J}^{\alpha}
$$

the following result was proved in [28].

Theorem 5.5. Let $\mathcal{G}$ be a Lie pseudo-group acting freely and regularly on $\mathcal{V}^{n^{\star}} \subset \mathrm{J}^{n^{\star}}$, where $n^{\star}$ is the order of freeness. Then a finite generating set for the algebra of differential invariants consists of

- the differential invariants $I_{\nu}=I_{\bar{\sigma}_{\nu}}$, where $\bar{\sigma}_{1}, \ldots, \bar{\sigma}_{l}$ form a Gröbner basis for the invariantized prolonged symbol submodule $\overline{\mathcal{J}}^{>n^{\star}}$, and, possibly,

- a finite number of additional differential invariants of order $\leq n^{\star}$. 
Remark 5.6. While Theorem 5.5 yields a generating set for the algebra of differential invariants, it certainly does not imply that this set is minimal. Unfortunately, there is, to this day, no algorithm for determining whether a generating set containing more than one differential invariant is minimal.

The algebraic considerations introduced above and the proof of Theorem 5.5 assumes the pseudo-group acts freely and regularly on a dense open subset $\mathcal{V}^{\infty} \subset \mathrm{J}^{\infty}$. In Example 3.13, this would correspond to the set of regular jets $\mathcal{V}_{1}^{\infty}$. For the other regular subbundles $\mathcal{V}_{2}^{\infty}, \ldots, \mathcal{V}_{5}^{\infty}$ of Example 3.13, we must incorporate into the algebraic constructions the dependencies among the submanifold jet coordinates introduced by the determining equations (3.26). To achieve this, a second identification of the one-forms on $\mathrm{J}^{\infty}$ with polynomials in $\mathcal{S}$ is introduced, [28]. This identification is given by

$$
d x^{i} \longleftrightarrow \widetilde{s}_{i}, \quad d u_{J}^{\alpha} \longleftrightarrow s_{J} S^{\alpha}
$$

Under the correspondence (5.12), the exterior differential of an $n^{\text {th }}$ order differential equation $E\left(x, u^{(n)}\right)=0$

$$
d E\left(x, u^{(n)}\right)=\sum_{i=1}^{p} \frac{\partial E}{\partial x^{i}}\left(x, u^{(n)}\right) d x^{i}+\sum_{\alpha=1}^{q} \sum_{\# J \leq n} \frac{\partial E}{\partial u_{J}^{\alpha}}\left(x, u^{(n)}\right) d u_{J}^{\alpha}=0
$$

at a submanifold jet $z^{(n)}=\left(x, u^{(n)}\right)$ can be identified with the parametrized polynomial

$$
\alpha\left(z^{(n)} ; \widetilde{s}, s, S\right)=\sum_{i=1}^{p} \frac{\partial E}{\partial x^{i}}\left(x, u^{(n)}\right) \widetilde{s}_{i}+\sum_{\alpha=1}^{q} \sum_{\# J \leq n} \frac{\partial E}{\partial u_{J}^{\alpha}}\left(x, u^{(n)}\right) s_{J} S^{\alpha} \in \mathcal{S}^{\leq n} .
$$

Definition 5.7. The symbol $\boldsymbol{\Sigma}\left(E\left(z^{(n)}\right)\right)$ of an $n^{\text {th }}$ order (non-zero) differential equation $E\left(z^{(n)}\right)$ $=E\left(x, u^{(n)}\right)=0$ at a submanifold jet $z^{(n)}=\left(x, u^{(n)}\right)$ consists of the highest order terms in the parametrized polynomial (5.13):

$$
\boldsymbol{\Sigma}\left[E\left(z^{(n)}\right)\right]=\mathbf{H}\left[\alpha\left(z^{(n)} ; \widetilde{s}, s, S\right)\right]=\sum_{\alpha=1}^{q} \sum_{\# J=n} \frac{\partial E}{\partial u_{J}^{\alpha}}\left(z^{(n)}\right) s_{J} S^{\alpha} \in \mathcal{S}^{n}
$$

Now, let $\mathcal{V}_{i}^{\infty} \subset \mathrm{J}^{\infty}$ be an invariant regular subbundle with determining system

$$
E_{i}^{(\infty)}\left(x, u^{(\infty)}\right)=0
$$

Since the system of differential equations (5.14) is formally integrable, its symbol at a fixed submanifold jet $z^{(\infty)}=\left(x, u^{(\infty)}\right)$

$$
\left.\mathcal{E}_{i}\right|_{z^{(\infty)}}=\boldsymbol{\Sigma}\left[E_{i}^{(\infty)}\left(z^{(\infty)}\right)\right]=\mathbf{H}\left[\left(T_{z^{(\infty)}} \mathcal{V}_{i}^{\infty}\right)^{\perp}\right]
$$

is a submodule of the submanifold jet module $\mathcal{S}$. Let $\left.\mathcal{M}_{i}\right|_{z(\infty)} \subset \mathcal{S}$ denote the monomial module generated by the leading monomials (with respect to a chosen term ordering on $\mathcal{S}$ ) of the symbol module $\left.\mathcal{E}_{i}\right|_{z(\infty)}$. We can assume, possibly by restricting to an open subset and employing $\delta$-regular coordinates (see Definition 6.4 below) that generically $\left.\mathcal{M}_{i}\right|_{z^{(\infty)}}=\mathcal{M}_{i}$ does not depend upon $z^{(\infty)} \in \mathcal{V}_{i}^{\infty}$. Let

$$
\mathcal{S}_{i}=\mathcal{S}^{-1} \oplus \operatorname{span}\left\{s_{J} S^{\alpha} \notin \mathcal{M}_{i}\right\} \subset \mathcal{S}
$$


denote the $i^{\text {th }}$ restricted submanifold jet module spanned by all monomials not in the monomial module $\mathcal{M}_{i}$ to which we add $\mathcal{S}^{-1}$. Applying standard Gaussian elimination we can construct a linear basis for the space $\left.\mathcal{E}_{i}\right|_{z(\infty)}$ of the form

$$
s_{I} S^{\beta}+\sum_{s_{J} S^{\alpha} \in \mathcal{S}_{i}^{n}} h_{\alpha}^{J}\left(z^{(n)}\right) s_{J} S^{\alpha} \quad \text { for all } \quad s_{I} S^{\beta} \in \mathcal{M}_{i}^{n}, \quad n \geq 0 .
$$

A similar statement holds for the subspace $\left(T_{z(\infty)} \mathcal{V}_{i}^{\infty}\right)^{\perp}$, where the sum in (5.15) now runs over all the monomials in $\mathcal{S}_{i}^{\leq n}$. Therefore, $\mathcal{S}_{i}$ is a fixed complement to the symbol module $\left.\mathcal{E}_{i}\right|_{z^{(\infty)}}$ and the annihilating subspace $\left(T_{z^{(\infty)}} \mathcal{V}_{i}^{\infty}\right)^{\perp}$ :

$$
\mathcal{S}=\mathcal{S}_{i} \oplus \mathcal{M}_{i}=\left.\mathcal{S}_{i} \oplus \mathcal{E}_{i}\right|_{z(\infty)}=\mathcal{S}_{i} \oplus\left(T_{z(\infty)} \mathcal{V}_{i}^{\infty}\right)^{\perp}
$$

We can thus identify the submodule

$$
\mathcal{S}_{i} \simeq \mathcal{S} /\left(\left.\mathcal{E}_{i}\right|_{z^{(\infty)}}\right) \simeq \mathcal{S} /\left(T_{z^{(\infty)}} \mathcal{V}_{i}^{\infty}\right)^{\perp} \simeq T_{z^{(\infty)}}^{*} \mathcal{V}_{i}^{\infty}
$$

with the dual space of $\mathcal{V}_{i}^{\infty}$ at the point $z^{(\infty)} \in \mathcal{V}_{i}^{\infty}$. Under the identification (5.12), the monomials $s_{J} S^{\alpha} \in \mathcal{S}_{i}$ indicate the parametric jet variables $u_{J}^{\alpha}$ on $\mathcal{V}_{i}^{\infty}$.

On each invariant regular subbundle $\mathcal{V}_{i}^{\infty}$, the algebraic constructions introduced before Remark 5.6 still hold provided the submanifold jet module $\mathcal{S}$ is replaced by the restricted submanifold jet module $\mathcal{S}_{i}$. Also, since $\mathcal{V}_{i}^{\infty}$ is $\mathcal{G}$-invariant, the determining system (5.14) is invariant and the invariantization of the algebraic constructions is well defined. In particular, the fundamental basis Theorem 5.5 still holds (with $n^{\star}$ replaced by $n_{i}^{\star}$ and $\overline{\mathcal{J}}^{>n^{\star}}$ replaced by $\overline{\mathcal{J}}_{i}^{>n_{i}^{\star}}$, where $\left.\mathcal{J}_{i}=\left(\beta^{*}\right)^{-1}\left(\left.\mathcal{I}\right|_{z}\right) \subset \widehat{\mathcal{S}}_{i}\right)$.

Example 5.8. Continuing Examples 3.13 and 4.13, since in the generic case $\mathcal{V}_{1}^{\infty}$ is a dense open subset of $\mathrm{J}^{\infty}$ the restricted submanifold jet module is the whole submanifold jet module: $\mathcal{S}_{1}=\mathcal{S}$. On the other hand, on $\mathcal{V}_{2}^{\infty}$ the differential constraint $Q_{P^{4}} \equiv 0$ implies that

$$
Q_{X^{i} U^{j} P^{k+4}} \equiv 0 \quad \text { or equivalently } \quad q_{x^{i} u^{j} p^{k+4}} \equiv 0, \quad i, j, k \geq 0 .
$$

The symbol associated to the determining system (5.16) is

$$
\mathcal{E}_{2}=\mathcal{M}_{2}=\operatorname{span}\left\{s_{x}^{i} s_{u}^{j} s_{p}^{k+4} S: i, j, k \geq 0\right\},
$$

and the corresponding restricted submanifold jet module is

$$
\mathcal{S}_{2}=\operatorname{span}\left\{\widetilde{s}_{x}, \widetilde{s}_{u}, \widetilde{s}_{p}\right\} \oplus \operatorname{span}\left\{s_{x}^{i} s_{u}^{j} s_{p}^{k} S: 0 \leq k \leq 3 \text { and } i, j \geq 0\right\} .
$$

Similar restricted submanifold jet modules $\mathcal{S}_{3}, \ldots, \mathcal{S}_{5}$ for the subbundles $\mathcal{V}_{3}^{\infty}, \ldots, \mathcal{V}_{5}^{\infty}$ can be obtained.

\subsection{Singular submanifold jets}

We now assume that $z^{(\infty)} \in \mathcal{S}_{i}^{\infty} \subset \mathrm{J}^{\infty}$ is a singular submanifold jet where the pseudo-group $\mathcal{G}$ acts regularly but not freely. As in the previous section, let $\mathcal{S}_{i}$ denote the restricted submanifold jet module associated with the regular invariant subbundle $\mathcal{S}_{i}^{\infty}$. Let

$$
\mathrm{j}_{\infty} \mathfrak{g}_{z(\infty)}=\left.\left.\operatorname{ker} \mathbf{p}\right|_{z^{(\infty)}} \cap \mathrm{J}^{\infty} \mathfrak{g}\right|_{z}, \quad z=\pi_{0}^{\infty}\left(z^{(\infty)}\right)
$$

be the vector field jets of the isotropy Lie algebra at $z^{(\infty)}$. Identifying $\left.\left(\mathrm{J}^{\infty} T_{M}\right)^{*}\right|_{z}$ with the symbol module $\mathcal{T}$, we introduce the isotropy annihilator vector space

$$
\left(\mathrm{j}_{\infty} \mathfrak{g}_{z(\infty)}\right)^{\perp}=\mathcal{T}_{i} \subset \mathcal{T} .
$$

To streamline the notation we have suppressed the dependence of $\mathcal{T}_{i}$ on the submanifold jet $z^{(\infty)} \in \mathcal{S}_{i}^{\infty}$. 
Proposition 5.9. Let $n_{i}^{\star}$ be the order of partial freeness of $\mathcal{G}$ on $\mathcal{S}_{i}^{\infty}$. Then for $n \geq n_{i}^{\star}$ and $z^{(n)} \in \mathcal{S}_{i}^{n}$,

$$
\mathbf{p}^{*}\left(\mathcal{S}_{i}^{\leq n}\right)+\left.\mathcal{L}^{\leq n}\right|_{z}=\mathcal{T}_{i}^{\leq n}
$$

Proof. For $z^{(n)} \in \mathcal{S}_{i}^{n}$ and $n \geq n_{i}^{\star}$ we have

$$
\mathrm{j}_{n} \mathfrak{g}_{z^{(n)}}=\left.\left.\operatorname{ker} \mathbf{p}^{(n)}\right|_{z^{(n)}} \cap \mathrm{J}^{n} \mathfrak{g}\right|_{z}=\left(\operatorname{rng}\left(\mathbf{p}^{(n)}\right)^{*}\right)^{\perp} \cap\left(\left.\mathcal{L}^{\leq n}\right|_{z}\right)^{\perp}=\left(\mathbf{p}^{*}\left(\mathcal{S}_{i}^{\leq n}\right)+\left.\mathcal{L}^{\leq n}\right|_{z}\right)^{\perp},
$$

from which (5.17) follows.

Lemma 5.4, essential to the proof of the fundamental basis Theorem 5.5, also holds at a singular submanifold jet.

Proposition 5.10. For $n>n_{i}^{\star}$ and $z^{(n)} \in \mathcal{S}_{i}^{n}$,

$$
\left.\mathcal{U}_{i}^{n}\right|_{z^{(n)}}=\left.\mathcal{J}_{i}^{n}\right|_{z^{(n)}}
$$

Proof. By induction, it suffices to prove (5.18) when $n=n_{i}^{\star}+1$. Let $\left.Q \in \mathcal{J}_{i}^{n_{i}^{\star}+1}\right|_{z^{\left(n_{i}^{\star}+1\right)}}$ and $P=\mathbf{p}^{*}(Q)$. By $(5.8)$ and (5.10)

$$
\mathbf{H}(P)=\mathbf{H}\left[\mathbf{p}^{*}(Q)\right]=\beta^{*}[\mathbf{H}(Q)]=\left.\beta^{*}(Q) \in \mathcal{I}^{n_{i}^{\star}+1}\right|_{z},
$$

and we conclude that there exists $Y \in \mathcal{T}^{\leq n_{i}^{\star}}$ such that $P+\left.Y \in \mathcal{L}^{n_{i}^{\star}+1}\right|_{z}$. Actually, we have that $Y \in \mathcal{T}_{i}^{\leq n_{i}^{\star}}$. To see this, we first observe that the formal integrability of the determining equations of the isotropy group $\mathcal{G}_{z^{\left(n_{i}^{\star}\right)}}^{\left(n_{\star}^{\star}\right)}$ implies that the projection $\mathcal{T}_{i}^{\leq n_{i}^{\star}+1} \rightarrow \mathcal{T}_{i}^{\leq n_{i}^{\star}}$ is surjective. Hence, by Proposition 5.9, since $P+Y$ and $P$ are in $\mathcal{T}_{i}^{\leq n_{i}^{\star}+1}$ we have that

$$
Y=(P+Y)-P \in \mathcal{T}_{i}^{\leq n_{i}^{\star}} .
$$

Now, let $U \in \mathcal{S}_{i}^{\leq n_{i}^{\star}}$ and $\left.V \in \mathcal{L}^{\leq n_{i}^{\star}}\right|_{z}$ such that $Y=\mathbf{p}^{*}(U)+V$, then

$$
\mathbf{p}^{*}(Q+U)=(P+Y)-\left.V \in \mathcal{L}^{\leq n_{i}^{\star}+1}\right|_{z} .
$$

Finally, equation (5.9) implies that $Q+\left.U \in \mathcal{Z}_{i}^{\leq n_{i}^{\star}+1}\right|_{z^{\left(n_{i}^{\star}+1\right)}}$.

Remark 5.11. The proof of Proposition 5.10 is essentially the same as [28, Lemma 5.5]. It is included to show that formal integrability of the determining equations of the isotropy group $\mathcal{G}_{z^{\left(n_{i}^{\star}\right)}}^{\left(n_{\star}^{\star}\right)}$ is essential for the proof to remain valid at a submanifold jet where the pseudo-group does not act freely. Due to Proposition 5.10, the constructive proof of the fundamental basis theorem given in [28] still holds at a singular submanifold jet $z^{(\infty)} \in \mathcal{S}_{i}^{\infty}$ (with the necessary algebraic modifications as in the regular case).

\section{Involutivity}

In Section 4.2 we showed via examples that in the appropriate geometrical setting, the pullback of the structure equations of an equivalence pseudo-group by a (partial) moving frame reproduces Cartan's moving frame results. In particular, in Example 4.15 we recovered the involutive structure equations (4.17). The aim of this final section is to complete Section 4.2 by recasting Cartan's involutivity test within the algebraic framework of Section 5 . Involutivity plays an essential role in the solution of equivalence problems, [26]. It guarantees that the equivalence map constructed by specifying its jets (or Taylor series coefficients) converges, and the Cartan characters give the "dimensional freedom" of the equivalence map in Theorem 4.11. The following exposition follows Seiler's book, [35]. 
Definition 6.1. Let

$$
\eta_{v}\left(z^{(n)} ; t, T\right)=\sum_{a=1}^{m} \sum_{\# B=n} h_{a ; v}^{B}\left(z^{(n)}\right) t_{B} T^{a}, \quad v=1, \ldots, \ell, \quad n \geq 1,
$$

be a basis for the degree $n$ homogeneous component of $\mathbf{H}\left(\mathcal{T}_{i}^{\leq n}\right)$. The symbol matrix

$$
\mathbf{T}_{i}^{n}=\left(h_{a ; v}^{B}\left(z^{(n)}\right)\right)
$$

is the $\ell \times m\left(\begin{array}{c}m+n-1 \\ n\end{array}\right)$ matrix where the entries of the $v^{\text {th }}$ row are given by the coefficients $h_{a ; v}^{B}\left(z^{(n)}\right)$ of the polynomial $\eta_{v}\left(z^{(n)} ; t, T\right)$.

To define the class of a symmetric multi-index $B=\left(b^{1}, \ldots, b^{n}\right)$ of order $\# B=n$ we rewrite the multi-index as $\widetilde{B}=\left(\widetilde{b}^{1}, \ldots, \widetilde{b}^{m}\right)$, where $\widetilde{b}^{a}$ is the number of occurrences of the integer $1 \leq a \leq m$ in $B=\left(b^{1}, \ldots, b^{n}\right)$.

Definition 6.2. The class of a multi-index $\widetilde{B}=\left(\widetilde{b}^{1}, \ldots, \widetilde{b}^{m}\right)$ is $\operatorname{cl} \widetilde{B}=\min \left\{a: \widetilde{b}^{a} \neq 0\right\}$.

Next, the columns of the symbol matrix $\mathbf{T}_{i}^{n}$ are ordered in such a way that the column $\left(h_{a ; 1}^{\widetilde{B}}, \ldots, h_{a ; \ell}^{\widetilde{B}}\right)^{T}$ is always to the left of the column $\left(h_{b ; 1}^{\widetilde{A}}, \ldots, h_{b ; \ell}^{\widetilde{A}}\right)^{T}$ if $\operatorname{cl} \widetilde{B}>\operatorname{cl} \widetilde{A}$. For two multi-indices with same class, the order of the columns does not matter. Once the columns of the symbol matrix are ordered, the matrix is put in row echelon form without performing any column permutations.

Definition 6.3. Let $\beta_{n}^{(a)}, a=1, \ldots, m$, be the number of pivots with class $1 \leq a \leq m$ of the row echelon form symbol matrix $\mathbf{T}_{i}^{n}$. The numbers $\beta_{n}^{(a)}$ are called the indices of $\mathbf{T}_{i}^{n}$.

Definition 6.3 depends on the chosen coordinate system and one must work with $\delta$-regular coordinate systems.

Definition 6.4. A coordinate system is said to be $\delta$-regular if the sum $\sum_{a=1}^{m} a \beta_{n}^{(a)}$ is maximal.

Any coordinate system can be transformed into a $\delta$-regular one with a linear transformation defined by a matrix coming from a Zariski open subset of $\mathbb{R}^{m \times m}$, [35].

Definition 6.5. The degree $n$ homogeneous component of $\mathbf{H}\left(\mathcal{T}_{i}^{\leq n}\right)$ is said to be involutive if the symbol matrix $\mathbf{T}_{i}^{n+1}$ of the degree $n+1$ homogeneous component of $\mathbf{H}\left(\mathcal{T}_{i}^{\leq n+1}\right)$ satisfies the algebraic equality

$$
\operatorname{rank} \mathbf{T}_{i}^{n+1}=\sum_{a=1}^{m} a \beta_{n}^{(a)}
$$

Definition 6.6. Let $n \geq 1$, the isotropy annihilator subbundle $\mathcal{T}_{i}^{\leq n}$ is in said to be involutive if the degree $n$ homogeneous component of $\mathbf{H}\left(\mathcal{T}_{i}^{\leq n}\right)$ is involutive and the projection $\mathcal{T}_{i}^{\leq n+1} \rightarrow \mathcal{T}_{i}^{\leq n}$ is surjective.

A standard result from the theory of involutivity guarantees that when $\mathcal{T}_{i}^{\leq n}$ becomes involutive then for all $k>n$ the isotropy annihilator subbundle $\mathcal{T}_{i}^{\leq k}$ remains involutive.

Definition 6.7. Assume $\mathcal{T}_{i}^{\leq n}$ is involutive with indices $\beta_{n}^{(a)}$. The Cartan characters $\alpha_{n}^{(a)}$ of $\mathcal{T}_{i}^{\leq n}$ are defined by

$$
\alpha_{n}^{(a)}=m\left(\begin{array}{c}
n+m-a-1 \\
n-1
\end{array}\right)-\beta_{n}^{(a)}, \quad 1 \leq a \leq m .
$$


Theorem 6.8. Let $\mathcal{T}_{i}^{\leq n}$ be involutive with Cartan characters $\alpha_{n}^{(a)}$. Then the isotropy group of the submanifold jet $z^{(\infty)}$ depends on $f_{a}$ arbitrary functions of a variables where the numbers $f_{a}$ are determined by the recursion relation

$$
\begin{aligned}
& f_{m}=\alpha_{n}^{(m)}, \\
& f_{a}=\alpha_{n}^{(a)}+\sum_{b=a+1}^{m} \frac{(a-1) !}{(m-1) !}\left(s_{b-a}^{(b-1)}(0) \alpha_{n}^{(b)}-s_{b-a}^{(b-1)}(n) f_{b}\right), \quad 1 \leq a \leq m-1,
\end{aligned}
$$

provided all $f_{a}$ are non-negative integers. The numbers $s_{b}^{(a)}(c)$ are the modified Stirling numbers defined by the identity

$$
(c+y+1)(c+y+2) \cdots(c+y+a)=\sum_{b=0}^{a} s_{a-b}^{(a)}(c) y^{b}
$$

for all non-negative integers $a, b, c$ and $a \geq b$. Here $y$ is an arbitrary variable.

Example 6.9. Continuing Example 4.15 we verify that the involutivity test (6.1) holds and compute the size of the isotropy group at a submanifold jet. First, we recall that for the contact pseudo-group (2.12) the normalized Maurer-Cartan forms, up to order 1, satisfy the linear relations

$$
\begin{aligned}
& \mu^{x} \equiv-\omega^{x}, \quad \mu^{u} \equiv-\omega^{u}, \quad \mu^{p} \equiv-\omega^{p}, \quad \mu_{P}^{u} \equiv \mu_{X}^{p} \equiv \mu_{Q}^{x} \equiv \mu_{Q}^{u} \equiv \mu_{Q}^{p} \equiv 0, \\
& \mu^{q}-\mu_{X}^{p} \equiv 0, \quad \mu_{X}^{u}-\mu^{p} \equiv 0, \quad \mu_{P}^{p}+\mu_{X}^{x}-\mu_{U}^{u} \equiv 0, \quad \mu_{Q}^{q}-\mu_{P}^{p}+\mu_{X}^{x} \equiv 0 .
\end{aligned}
$$

To show that the degree 1 homogeneous component of $\mathbf{H}\left(\mathcal{T}_{1}^{\leq 1}\right)$ is involutive, we first note that via the lift map (3.8) the vector field jet $\zeta_{B}^{a}$ can be identified with the Maurer-Cartan form $\mu_{B}^{a}$ so that we have the identification

$$
\mu_{B}^{a} \longleftrightarrow \zeta_{B}^{a} \longleftrightarrow t_{B} T^{a}
$$

Secondly, since the invariantization of the algebraic constructions introduced in Section 5 coincide with their progenitor when restricted to a cross-section, we can use the linear relations among the normalized Maurer-Cartan forms (6.2) to verify the involutivity test (6.1) at a submanifold jet on the cross-section defining a partial moving frame. Using the correspondence (6.3), the isotropy annihilator polynomials associated to (6.2) are

$$
\begin{aligned}
& T^{x}, \quad T^{u}, \quad T^{p}, \quad t_{p} T^{u}, \quad t_{x} T^{p}, \quad t_{q} T^{x}, \quad t_{q} T^{u}, \quad t_{q} T^{p}, \\
& T^{q}-t_{x} T^{p}, \quad t_{x} T^{u}-T^{p}, \quad t_{p} T^{p}+t_{x} T^{x}-t_{u} T^{u}, \quad t_{q} T^{q}-t_{p} T^{p}+t_{x} T^{x}
\end{aligned}
$$

Writing the order 1 symbol matrix $\mathbf{T}^{1}$ we obtain

$$
\begin{aligned}
& t_{q} T^{q} \quad t_{q} T^{p} \quad t_{q} T^{u} \quad t_{q} T^{x} \quad t_{x} T^{p} \quad t_{x} T^{u} \quad t_{x} T^{x} \quad t_{p} T^{p} \quad t_{p} T^{u} \quad t_{p} T^{x} \quad t_{u} T^{p} \quad t_{u} T^{u} t_{u} T^{x} \\
& \left(\begin{array}{ccccccccccccc}
1 & 0 & 0 & 0 & 0 & 0 & -1 & 1 & 0 & 0 & 0 & 0 & 0 \\
0 & 1 & 0 & 0 & 0 & 0 & 0 & 0 & 0 & 0 & 0 & 0 & 0 \\
0 & 0 & 1 & 0 & 0 & 0 & 0 & 0 & 0 & 0 & 0 & 0 & 0 \\
0 & 0 & 0 & 1 & 0 & 0 & 0 & 0 & 0 & 0 & 0 & 0 & 0 \\
0 & 0 & 0 & 0 & 1 & 0 & 0 & 0 & 0 & 0 & 0 & 0 & 0 \\
0 & 0 & 0 & 0 & -1 & 0 & 0 & 0 & 0 & 0 & 0 & 0 & 0 \\
0 & 0 & 0 & 0 & 0 & 1 & 0 & 0 & 0 & 0 & 0 & 0 & 0 \\
0 & 0 & 0 & 0 & 0 & 0 & 1 & 1 & 0 & 0 & 0 & -1 & 0 \\
0 & 0 & 0 & 0 & 0 & 0 & 0 & 0 & 1 & 0 & 0 & 0 & 0
\end{array}\right)
\end{aligned}
$$


and find that the indices of the matrix are $\beta_{1}^{(4)}=4, \beta_{1}^{(3)}=3, \beta_{1}^{(2)}=1$ and $\beta_{1}^{(1)}=0$. At order 2 , the rank of the symbol matrix $\mathbf{T}^{2}$ is $27=4 \beta_{1}^{(4)}+3 \beta_{1}^{(3)}+2 \beta_{1}^{(2)}+\beta_{1}^{(1)}$ which satisfies the involutivity test. The corresponding Cartan characters are $\alpha_{1}^{(4)}=\alpha_{1}^{(3)}=0, \alpha_{1}^{(2)}=2, \alpha_{1}^{(1)}=3$, and we conclude that the isotropy group involves two arbitrary analytic functions, each depending on two variables. In other words, the general contact transformation between two second order ordinary differential equations depends on two arbitrary functions of two variables. This is in accordance with Cartan's involutivity test based on the theory of exterior differential systems, [26, Example 11.10].

Example 6.10. In this final example we consider the simultaneous equivalence of a two-form and a vector field on $\mathbb{R}^{3}$, [11]. This example is interesting as the solution admits a branch with an infinite-dimensional isotropy group and an essential invariant. Let

$$
\Omega=a(x, y, z) d x \wedge d y+b(x, y, z) d x \wedge d z+c(x, y, z) d y \wedge d z, \quad a(x, y, z) \neq 0,
$$

be a non-vanishing two-form, and

$$
\mathbf{v}=e(x, y, z) \frac{\partial}{\partial x}+f(x, y, z) \frac{\partial}{\partial y}+g(x, y, z) \frac{\partial}{\partial z}, \quad g(x, y, z) \neq 0
$$

a non-zero vector field on $\mathbb{R}^{3}$. If

$$
\begin{aligned}
& \bar{\Omega}=A(X, Y, Z) d X \wedge d Y+B(X, Y, Z) d X \wedge d Z+C(X, Y, Z) d Y \wedge d Z, \quad A(X, Y, Z) \neq 0, \\
& \overline{\mathbf{v}}=E(X, Y, Z) \frac{\partial}{\partial X}+F(X, Y, Z) \frac{\partial}{\partial Y}+G(X, Y, Z) \frac{\partial}{\partial Z}, \quad G(X, Y, Z) \neq 0,
\end{aligned}
$$

is another set of non-vanishing two-form and vector field on $\mathbb{R}^{3}$, the map

$$
\Phi: X=\phi(x, y, z), Y=\beta(x, y, z), Z=\alpha(x, y, z) \in \mathcal{D}\left(\mathbb{R}^{3}\right),
$$

is a local equivalence if it satisfies

$$
\Phi^{*}(\bar{\Omega})=\Omega \quad \text { and } \quad d \Phi^{-1}(\overline{\mathbf{v}})=\mathbf{v} .
$$

The equivalence problem splits in two cases; either $\mathbf{v}\lrcorner \Omega=0$ or $\mathbf{v}\lrcorner \Omega \neq 0$. In the following we consider the case $\mathbf{v}\lrcorner \Omega=0$. This imposes the restrictions

$$
e(x, y, z)=\frac{g(x, y, z) c(x, y, z)}{a(x, y, z)} \quad \text { and } \quad f(x, y, z)=-\frac{g(x, y, z) b(x, y, z)}{a(x, y, z)}
$$

on the coefficients of vector field (6.5) (and similar constraints on the coefficients of $\overline{\mathbf{v}}$ ). In local coordinates, the equivalence criterions (6.6) yield the transformation rules

$$
\begin{aligned}
& A\left(\phi_{x} \beta_{y}-\beta_{x} \phi_{y}\right)+B\left(\phi_{x} \alpha_{y}-\alpha_{x} \phi_{y}\right)+C\left(\beta_{x} \alpha_{y}-\alpha_{x} \beta_{y}\right)=a, \\
& A\left(\phi_{x} \beta_{z}-\beta_{x} \phi_{z}\right)+B\left(\phi_{x} \alpha_{z}-\alpha_{x} \phi_{z}\right)+C\left(\beta_{x} \alpha_{z}-\alpha_{x} \beta_{z}\right)=b, \\
& A\left(\phi_{y} \beta_{z}-\beta_{y} \phi_{z}\right)+B\left(\phi_{y} \alpha_{z}-\alpha_{y} \phi_{z}\right)+C\left(\beta_{y} \alpha_{z}-\alpha_{y} \beta_{z}\right)=c, \\
& G=\frac{g}{a}\left(c \alpha_{x}-b \alpha_{y}+a \alpha_{z}\right),
\end{aligned}
$$

for the components of the two-form (6.4) and the vector field (6.5). The infinitesimal generator corresponding to the Lie pseudo-group action (6.7) is

$$
\mathbf{w}=\xi(x, y, z) \frac{\partial}{\partial x}+\eta(x, y, z) \frac{\partial}{\partial y}+\tau(x, y, z) \frac{\partial}{\partial z}+\left[c \tau_{x}-a\left(\xi_{x}+\eta_{y}\right)-b \tau_{y}\right] \frac{\partial}{\partial a}
$$




$$
-\left[a \eta_{z}+b\left(\xi_{x}+\tau_{z}\right)+c \eta_{x}\right] \frac{\partial}{\partial b}+\left[a \xi_{z}-b \xi_{y}-c\left(\eta_{y}+\tau_{z}\right)\right] \frac{\partial}{\partial c}+\frac{g}{a}\left[c \tau_{x}-b \tau_{y}+a \tau_{z}\right] \frac{\partial}{\partial g},
$$

where $\xi(x, y, z), \eta(x, y, z)$ and $\tau(x, y, z)$ are arbitrary analytic functions. The rank of the Lie matrix, [27], of the first prolongation $\mathbf{w}^{(1)}$ reveals that the orbits of the first order prolonged action are of codimension one in $J^{1}$. Hence the equivalence pseudo-group admits a first order differential invariant:

$$
I=\frac{g}{a}\left(a_{z}-b_{y}+c_{x}\right)
$$

Implementing the moving frame algorithm, the analysis of the recurrence relations reveals that it is possible to make the "universal" normalizations

$$
\begin{aligned}
& X=Y=Z=0, \quad A=G=1, \quad B_{X^{i} Y^{j} Z^{k}}=C_{X^{i} Y^{j} Z^{k}}=0, \quad i+j+k \geq 0, \\
& G_{X^{i} Y^{j} Z^{k}}=0, \quad i+j+k \geq 1, \quad A_{X^{i} Y^{j}}=0, \quad i+j \geq 1,
\end{aligned}
$$

leading to the normalization of the Maurer-Cartan forms

$$
\begin{aligned}
& \mu=\lambda(\xi), \quad \nu=\lambda(\eta) \quad \alpha=\lambda(\tau), \quad \nu_{X^{i} Y^{j+1}}=\lambda\left(\eta_{x^{i} y^{j+1}}\right), \\
& \nu_{X^{i} Y^{j} Z^{k+1}}=\lambda\left(\eta_{x^{i} y^{j} z^{k+1}}\right), \quad \mu_{X^{i} Y^{j} Z^{k+1}}=\lambda\left(\xi_{x^{i} y^{j} z^{k+1}}\right), \quad \alpha_{X^{i} Y^{j} Z^{k+1}}=\lambda\left(\tau_{x^{i} y^{j} z^{k+1}}\right),
\end{aligned}
$$

$i, j, k \geq 0$. With the normalizations (6.9), the invariant (6.8) corresponds to

$$
I=A_{Z}=\iota\left(a_{z}\right),
$$

and the invariant coframe $\omega=\left\{\omega^{x}, \omega^{y}, \omega^{z}\right\}=\iota\{d x, d y, d z\}$ is such that $\Omega=\omega^{x} \wedge \omega^{y}$, and $\mathbf{v}\lrcorner \omega^{z}=1$. Geometrically, the invariant (6.8) measures the obstruction of $\Omega$ to being closed:

$$
d \Omega=I \omega^{x} \wedge \omega^{y} \wedge \omega^{z} .
$$

After making the normalizations (6.9), the only remaining partially normalized invariants are

$$
A_{X^{i} Y^{j} Z^{k+1}}, \quad i, j, k \geq 0 .
$$

Up to order 2, the recurrence relations for these invariants are

$$
\begin{aligned}
d A_{Z} \equiv & A_{X Z} \omega^{x}+A_{Y Z} \omega^{y}+\left(A_{Z Z}-A_{Z}^{2}\right) \omega^{z} \\
d A_{X Z} \equiv & A_{X X Z} \omega^{x}+A_{X Y Z} \omega^{y}+\left(A_{X Z Z}-2 A_{Z} A_{X Z}\right) \omega^{z} \\
& -A_{Y Z} \nu_{X}-A_{X Z} \mu_{X}-\left(A_{Z Z}-A_{Z}^{2}\right) \alpha_{X}, \\
d A_{Y Z} \equiv & A_{X Y Z} \omega^{x}+A_{Y Y Z} \omega^{y}+\left(A_{Y Z Z}-3 A_{Z} A_{Y Z}\right) \omega^{z} \\
& +A_{Y Z} \mu_{X}-A_{X Z} \mu_{Y}-\left(A_{Z Z}-A_{Z}^{2}\right) \alpha_{Y}, \\
d A_{Z Z} \equiv & A_{X Z Z} \omega^{x}+A_{Y Z Z} \omega^{y}+\left(A_{Z Z Z}-A_{Z} A_{Z Z}\right) \omega^{z} .
\end{aligned}
$$

At this stage, the equivalence problem splits in two branches:

Case 1: $A_{Z}$ is constant.

Case 2: $A_{Z}$ is not constant.

In Case 1, when $A_{Z} \equiv c$ is constant, the recurrence relations (6.12), and the higher order ones, imply that the remaining partially normalized invariants (6.11) are constant. For example, from (6.12) we have that

$$
\begin{aligned}
& A_{Z} \equiv c, \quad A_{X Z} \equiv A_{Y Z} \equiv 0, \quad A_{Z Z} \equiv c^{2}, \\
& A_{X X Z} \equiv A_{X Y Z} \equiv Z_{X Z Z} \equiv A_{Y Y Z} \equiv A_{Y Z Z} \equiv 0, \quad A_{Z Z Z} \equiv c^{3} .
\end{aligned}
$$


Hence, in this case, there are no further normalizations possible. Up to order 2, the normalized Maurer-Cartan forms are

$$
\begin{aligned}
& \mu \equiv-\omega^{x}, \quad \nu \equiv-\omega^{y}, \quad \alpha=-\omega^{z}, \quad \nu_{Y} \equiv-\mu_{X}+A_{Z} \omega^{z}, \quad \nu_{Z} \equiv \mu_{Z} \equiv \alpha_{Z} \equiv 0 \\
& \nu_{X Y} \equiv-\mu_{X X}-A_{Z} \alpha_{X}-A_{X Z} \omega^{z}, \quad \nu_{Y Y} \equiv-\mu_{X Y}-A_{Z} \alpha_{Y}+A_{Y Z} \omega^{z} \\
& \nu_{Z X} \equiv \nu_{Z Y} \equiv \nu_{Z Z} \equiv \mu_{X Z} \equiv \mu_{Y Z} \equiv \mu_{Z Z} \equiv \alpha_{X Z} \equiv \alpha_{Y Z} \equiv \alpha_{Z Z} \equiv 0 .
\end{aligned}
$$

The order 1 isotropy annihilator polynomials associated to (6.13) are

$$
t_{y} T^{y}+t_{x} T^{x}, \quad t_{z} T^{y}, \quad t_{z} T^{x}, \quad t_{z} T^{z}
$$

and the indices of the reduced ${ }^{3}$ symbol matrix $\mathbf{T}^{1}$ are $\beta_{1}^{(3)}=3, \beta_{1}^{(2)}=1, \beta_{1}^{(1)}=0$. At order 2 , the rank of the reduced symbol matrix $\mathbf{T}^{2}$ is $11=3 \beta_{1}^{(3)}+2 \beta_{1}^{(2)}+\beta_{1}^{(1)}$, and Cartan's involutivity test is satisfied. The corresponding Cartan characters are $\alpha_{1}^{(3)}=0, \alpha_{1}^{(2)}=2$, and $\alpha_{1}^{(1)}=3$.

The structure equations for the horizontal coframe $\boldsymbol{\omega}=\left\{\omega^{x}, \omega^{y}, \omega^{z}\right\}$ are

$$
\begin{aligned}
d \omega^{x} & \equiv \mu_{X} \wedge \omega^{x}+\mu_{Y} \wedge \omega^{y} \\
d \omega^{y} & \equiv \nu_{X} \wedge \omega^{x}-\mu_{X} \wedge \omega^{y}-c \omega^{y} \wedge \omega^{z} \\
d \omega^{z} & \equiv \alpha_{X} \wedge \omega^{x}+\alpha_{Y} \wedge \omega^{y} .
\end{aligned}
$$

These equations are equivalent to those obtained with Cartan's method, [26, equation (11.29)]. The correspondence is given by

$$
\begin{aligned}
& \theta^{1} \leftrightarrow \omega^{x}, \quad \theta^{2} \leftrightarrow \omega^{y}, \quad \theta^{3} \leftrightarrow \omega^{z}, \quad \alpha^{1} \leftrightarrow \mu_{X}, \quad \alpha^{2} \leftrightarrow \mu_{Y}, \quad \alpha^{3} \leftrightarrow \nu_{X}, \\
& \beta^{1} \leftrightarrow \alpha_{X}, \quad \beta^{2} \leftrightarrow \alpha_{Y}, \quad T \leftrightarrow A_{Z} .
\end{aligned}
$$

Moving to Case 2, where $A_{Z}$ is not constant, two sub-branches must be considered:

Case 2.1: $A_{Z Z} \equiv A_{Z}^{2}$.

Case 2.2: $A_{Z Z} \not \equiv A_{Z}^{2}$.

In Case 2.1, since $d A_{Z} \not \equiv 0$, the recurrence relation for $A_{Z}$ in (6.12) implies, that $\left(A_{X Z}, A_{Y Z}\right)$ $\not \equiv(0,0)$. Assuming $A_{X Z}>0$, we set

$$
A_{X Z}=1, \quad A_{Y Z}=0, \quad A_{X^{i} Y^{j} Z}=0, \quad i+j \geq 2,
$$

and normalize the Maurer-Cartan forms $\mu_{X^{i} Y^{j}}, i+j \geq 1$. The first recurrence relation in (6.12) then reduces to

$$
d A_{Z} \equiv \omega^{x}
$$

Substituting the equality $A_{Z Z}=A_{Z}^{2}$ into the last equation of (6.12) we obtain

$$
2 A_{Z} \omega^{x}=A_{X Z Z} \omega^{x}+A_{Y Z Z} \omega^{y}+\left(A_{Z Z Z}-A_{Z}^{3}\right) \omega^{z},
$$

which means that

$$
A_{X Z Z} \equiv 2 A_{Z}, \quad A_{Y Z Z} \equiv 0, \quad A_{Z Z Z} \equiv A_{Z}^{3} .
$$

\footnotetext{
${ }^{3}$ Notice that in (6.14) we have omitted the polynomials in $T^{a}, T^{b}, T^{c}$ and $T^{g}$ associated to the dependent variables of the problem as in this example these do not play an essential role in the involutivity test. The corresponding Cartan characters are all zero.
} 
Similarly, all higher order invariants $A_{X^{i} Y^{j} Z^{k+2}}=F_{i j k}\left(A_{Z}\right)$ are function of the invariant (6.10). Hence, no further normalizations are possible. Up to order 2, the normalized Maurer-Cartan forms are

$$
\begin{aligned}
& \mu \equiv-\omega^{x}, \quad \nu \equiv-\omega^{y}, \quad \alpha=-\omega^{z}, \quad \nu_{Y} \equiv A_{Z} \omega^{z} \\
& \nu_{Z} \equiv \mu_{Z} \equiv \alpha_{Z} \equiv \mu_{X} \equiv \mu_{Y} \equiv 0, \quad \nu_{X Y} \equiv-A_{Z} \alpha_{X}-\omega^{z}, \quad \nu_{Y Y} \equiv-A_{Z} \alpha_{Y}, \\
& \nu_{Z X} \equiv \nu_{Z Y} \equiv \nu_{Z Z} \equiv \mu_{X Z} \equiv \mu_{Y Z} \equiv \mu_{Z Z} \equiv \alpha_{X Z} \equiv \alpha_{Y Z} \equiv \alpha_{Z Z} \equiv \mu_{X X} \equiv \mu_{X Y} \equiv \mu_{Y Y} \equiv 0 .
\end{aligned}
$$

The order 1 isotropy annihilator polynomials associated with (6.16) are

$$
t_{y} T^{y}, \quad t_{z} T^{y}, \quad t_{z} T^{x}, \quad t_{z} T^{z}, \quad t_{x} T^{x}, \quad t_{y} T^{x}
$$

and the indices of the reduced symbol matrix $\mathbf{T}^{1}$ are $\beta_{1}^{(3)}=3, \beta_{1}^{(2)}=2, \beta_{1}^{(1)}=1$. At order 2 , the rank of the reduced symbol matrix $\mathbf{T}^{2}$ is $14=3 \beta_{1}^{(3)}+2 \beta_{1}^{(2)}+\beta_{1}^{(1)}$, which satisfies Cartan's involutivity test. The corresponding Cartan characters are $\alpha_{1}^{(3)}=0, \alpha_{1}^{(2)}=1$, and $\alpha_{1}^{(1)}=2$. Finally, the structure equations for the horizontal coframe $\boldsymbol{\omega}=\left\{\omega^{x}, \omega^{y}, \omega^{z}\right\}$ are

$$
d \omega^{x} \equiv 0, \quad d \omega^{y} \equiv \nu_{X} \wedge \omega^{x}-A_{Z} \omega^{y} \wedge \omega^{z}, \quad d \omega^{z} \equiv \alpha_{X} \wedge \omega^{x}+\alpha_{Y} \wedge \omega^{y} .
$$

Once again, using the correspondence (6.15) we recover the structure equations obtained via Cartan's method, [26, p. 370]. Case 2.2 is treated in a similar fashion. The complete analysis, based on Cartan's equivalence method, can be found in [11].

\section{Acknowledgments}

I am grateful to the three anonymous referees for their comments and suggestions which helped improve the paper. I would also like to thank Abraham Smith, Niky Kamran and Robert Milson for their suggestions and stimulating discussions, and Peter Olver for his comments on an early draft of the manuscript. This project was supported by an AARMS Postdoctoral Fellowship and a NSERC of Canada Postdoctoral Fellowship.

\section{References}

[1] Akivis M.A., Rosenfeld B.A., Élie Cartan (1869-1951), Translations of Mathematical Monographs, Vol. 123, Amer. Math. Soc., Providence, RI, 1993.

[2] Bryant R.L., Chern S.S., Gardner R.B., Goldschmidt H.L., Griffiths P.A., Exterior differential systems, Mathematical Sciences Research Institute Publications, Vol. 18, Springer-Verlag, New York, 1991.

[3] Cartan E., La structure des groupes infinis, in Oeuvres Complètes, Part. II, Vol. 2, Gauthier-Villars, Paris, 1953, 1335-1384.

[4] Cartan E., Sur la structure des groupes infinis de transformations, in Oeuvres Complètes, Part. II, Vol. 2, Gauthier-Villars, Paris, 1953, 571-714.

[5] Cartan E., Sur les variétés à connexion projective, in Oeuvres Complètes, Part. III, Vol. 1, Gauthier-Villars, Paris, 1955, 825-861.

[6] Cheh J., Olver P.J., Pohjanpelto J., Algorithms for differential invariants of symmetry groups of differential equations, Found. Comput. Math. 8 (2008), 501-532.

[7] Cheh J., Olver P.J., Pohjanpelto J., Maurer-Cartan equations for Lie symmetry pseudogroups of differential equations, J. Math. Phys. 46 (2005), 023504, 11 pages.

[8] Cox D., Little J., O'Shea D., Ideals, varieties, and algorithms. An introduction to computational algebraic geometry and commutative algebra, 3rd ed., Undergraduate Texts in Mathematics, Springer, New York, 2007.

[9] Fels M., Olver P.J., Moving coframes. II. Regularization and theoretical foundations, Acta Appl. Math. 55 (1999), 127-208. 
[10] Gardner R.B., The method of equivalence and its applications, CBMS-NSF Regional Conference Series in Applied Mathematics, Vol. 58, Society for Industrial and Applied Mathematics (SIAM), Philadelphia, PA, 1989.

[11] Gardner R.B., Shadwick W.F., An equivalence problem for a two-form and a vector field on $\mathbf{R}^{3}$, in Differential Geometry, Global Analysis, and Topology (Halifax, NS, 1990), CMS Conf. Proc., Vol. 12, Amer. Math. Soc., Providence, RI, 1991, 41-50.

[12] Guillemin V., Sternberg S., Deformation theory of pseudogroup structures, Mem. Amer. Math. Soc. 64 (1966), 80 pages.

[13] Hubert E., Differential invariants of a Lie group action: syzygies on a generating set, J. Symbolic Comput. 44 (2009), 382-416, arXiv:0710.4318.

[14] Johnson H.H., Classical differential invariants and applications to partial differential equations, Math. Ann. 148 (1962), 308-329.

[15] Kamran N., Contributions to the study of the equivalence problem of Élie Cartan and its applications to partial and ordinary differential equations, Acad. Roy. Belg. Cl. Sci. Mém. Collect. $8^{\circ}$ (2) 45 (1989), no. 7, 122 pages.

[16] Kogan I.A., Olver P.J., Invariant Euler-Lagrange equations and the invariant variational bicomplex, Acta Appl. Math. 76 (2003), 137-193.

[17] Kruglikov B., Lychagin V., Global Lie-Tresse theorem, arXiv:1111.5480.

[18] Kruglikov B., Lychagin V., Invariants of pseudogroup actions: homological methods and finiteness theorem, Int. J. Geom. Methods Mod. Phys. 3 (2006), 1131-1165, math.DG/0511711.

[19] Kumpera A., Invariants différentiels d'un pseudogroupe de Lie. I, J. Differential Geometry 10 (1975), 289345.

[20] Kuranishi M., On the local theory of continuous infinite pseudo groups. I, Nagoya Math. J 15 (1959), 225-260.

[21] Lie S., Klassifikation und Integration von gewöhnlichen Differentialgleichungen zwischen $x, y$, die eine Gruppe von Transformationen gestatten I-IV, in Gesammelte Abhandlungen, Vol. 5, B.G. Teubner, Leipzig, 1924, 240-310, 362-427, 432-448.

[22] Lie S., Scheffers G., Vorlesungen über Continuierliche Guppen mit Geometrischen und Anderen Anwendungen, B.G. Teubner, Leipzig, 1893.

[23] Mackenzie K., Lie groupoids and Lie algebroids in differential geometry, London Mathematical Society Lecture Note Series, Vol. 124, Cambridge University Press, Cambridge, 1987.

[24] Milson R., Valiquette F., Point equivalence of second-order odes: maximal classifying order, arXiv:1208.1014.

[25] Muñoz J., Muriel F.J., Rodríguez J., On the finiteness of differential invariants, J. Math. Anal. Appl. 284 (2003), 266-282.

[26] Olver P.J., Equivalence, invariants, and symmetry, Cambridge University Press, Cambridge, 1995.

[27] Olver P.J., Moving frames and singularities of prolonged group actions, Selecta Math. (N.S.) 6 (2000), 41-77.

[28] Olver P.J., Pohjanpelto J., Differential invariant algebras of Lie pseudo-groups, Adv. Math. 222 (2009), 1746-1792.

[29] Olver P.J., Pohjanpelto J., Maurer-Cartan forms and the structure of Lie pseudo-groups, Selecta Math. (N.S.) 11 (2005), 99-126.

[30] Olver P.J., Pohjanpelto J., Moving frames for Lie pseudo-groups, Canad. J. Math. 60 (2008), 1336-1386.

[31] Olver P.J., Pohjanpelto J., Persistence of freeness for Lie pseudogroup actions, Ark. Mat. 50 (2012), 165-182, arXiv:0912.4501.

[32] Olver P.J., Recursive moving frames, Results Math. 60 (2011), 423-452.

[33] Olver P.J., Pohjanpelto J., Valiquette F., On the structure of Lie pseudo-groups, SIGMA 5 (2009), 077, 14 pages, arXiv:0907.4086.

[34] Pohjanpelto J., Reduction of exterior differential systems with infinite dimensional symmetry groups, BIT Num. Math. 48 (2008), 337-355.

[35] Seiler W.M., Involution. The formal theory of differential equations and its applications in computer algebra, Algorithms and Computation in Mathematics, Vol. 24, Springer-Verlag, Berlin, 2010.

[36] Shemyakova E., Mansfield E.L., Moving frames for Laplace invariants, in Proceedings ISSAC 2008, Editor D. Jeffrey, ACM, New York, 2008, 295-302. 
[37] Singer I.M., Sternberg S., The infinite groups of Lie and Cartan. I. The transitive groups, J. Analyse Math. 15 (1965), 1-114.

[38] Thompson R., Valiquette F., Group foliation of differential equations using moving frames, Preprint, Dalhousie University, 2012.

[39] Thompson R., Valiquette F., On the cohomology of the invariant Euler-Lagrange complex, Acta Appl. Math. 116 (2011), 199-226.

[40] Tresse A., Détermination des invariants ponctuels de l'équation différentielle ordinaire de second ordre $y^{\prime \prime}=\omega\left(x, y, y^{\prime}\right)$, Hirzel, Leipzig, 1896.

[41] Tresse A., Sur les invariants différentiels des groupes continus de transformations, Acta Math. 18 (1894), $1-88$.

[42] Valiquette F., Structure equations of Lie pseudo-groups, J. Lie Theory 18 (2008), 869-895. 